ISSN 2075-1729

www.mdpi.com/journal/life

Article

\title{
Theory of the Origin, Evolution, and Nature of Life
}

\section{Erik D. Andrulis}

Department of Molecular Biology and Microbiology, Case Western Reserve University School of Medicine, Wood Building, W212, Cleveland,OH 44106, USA; E-Mail: exa32@case.edu; Tel.: +1-216-368-0261; Fax: +1-216-368-3055

Received: 15 November 2011; in revised form: 10 December 2011 / Accepted: 13 December 2011 / Published: 23 December 2011

\begin{abstract}
Life is an inordinately complex unsolved puzzle. Despite significant theoretical progress, experimental anomalies, paradoxes, and enigmas have revealed paradigmatic limitations. Thus, the advancement of scientific understanding requires new models that resolve fundamental problems. Here, I present a theoretical framework that economically fits evidence accumulated from examinations of life. This theory is based upon a straightforward and non-mathematical core model and proposes unique yet empirically consistent explanations for major phenomena including, but not limited to, quantum gravity, phase transitions of water, why living systems are predominantly CHNOPS (carbon, hydrogen, nitrogen, oxygen, phosphorus, and sulfur), homochirality of sugars and amino acids, homeoviscous adaptation, triplet code, and DNA mutations. The theoretical framework unifies the macrocosmic and microcosmic realms, validates predicted laws of nature, and solves the puzzle of the origin and evolution of cellular life in the universe.
\end{abstract}

Keywords: quantum; gyre; emergence; thermodynamics; singularity; natural law; adaptation; learning and memory

\section{Introduction}

How life abides by the second law of thermodynamics yet evolutionarily complexifies and maintains its intrinsic order is a fundamental mystery in physics, chemistry, and biology [1]. Solving this problem requires an interdisciplinary knowledge and an awareness of conventional theories, especially those related to the origin and evolution of life. Rather than give a comprehensive literature review, I introduce a handful of these ideas and point out their limitations. 
The panspermia hypothesis has many forms, some of which suggest that life started elsewhere in the universe and arrived on Earth by cometary, meteoric, or planetary delivery [2,3]. The problem with this group of models is that it does not, in an empirically complete and consistent manner, explain the molecular origin of the first cell and hence avoids the issue in need of solution. The primordial soup hypothesis, also know as the Oparin-Haldane model, posits that during the early evolution of the Earth, a reducing atmosphere provided the correct environment for the formation of basic organic compounds [4,5]. Though the soup model has matured in recent decades, it has difficulty explaining the exact conditions of the early Earth atmosphere and the manner and order of emergence of polymeric systems. In the iron-sulfur world theory, primitive life is assumed to have started at deep-sea hydrothermal vents as a mineral base; redox reactions provided the chemical energy to drive the emergence of cellular life [6]. However, this model does not explain the origin of genetic information, membrane systems, or the complexification or diversity of cellular structure. Finally, the RNA (ribonucleic acid) world hypothesis posits that ribonucleotide-based genetic systems evolved prior to protein and deoxyribonucleic acid (DNA). This hypothesis does not fit well with the central dogma and is unable to resolve precisely how the translation apparatus, genetic code, and biometabolic pathways evolved [7-9]. In short, no consensus model for life has emerged.

Now, therefore, to know what life is and how life works, scientists need a scientifically accurate theory. The aim of a scientific theory is to construct a formal structure - in which the natural world is being modeled - to explain, predict, and control systems, events, and objects. Insofar as the physical, chemical, and biological sciences are true, physical reality and life itself thus reflexively model such a scientific theory; tautologically, the natural world subsumes said theory. Several investigators have detailed what would be required of a unifying bioscientific theory [1,10-24]. The correct theory would be expected to not only explain how the living cell works now, but also to provide insight into the evolution of life on Earth.

In the theory proposed herein, I use the heterodox yet simple gyre - a spiral, vortex, whorl, or similar circular pattern — as a core model for understanding life. Because many elements of the gyre model (gyromodel) are alien, I introduce neologisms and important terms in bold italics to identify them; a theoretical lexicon is presented in Table 1. The central idea of this theory is that all physical reality, stretching from the so-called inanimate into the animate realm and from micro- to meso- to macrocosmic scales, can be interpreted and modeled as manifestations of a single geometric entity, the gyre. This entity is attractive because it has life-like characteristics, undergoes morphogenesis, and is responsive to environmental conditions. The gyromodel depicts the spatiotemporal behavior and properties of elementary particles, celestial bodies, atoms, chemicals, molecules, and systems as quantized packets of information, energy, and/or matter that oscillate between excited and ground states around a singularity. The singularity, in turn, modulates these states by alternating attractive and repulsive forces. The singularity itself is modeled as a gyre, thus evincing a thermodynamic, fractal, and nested organization of the gyromodel. In fitting the scientific evidence from quantum gravity to cell division, this theory arrives at an understanding of life that questions traditional beliefs and definitions. 
Table 1. Gyromodel Lexicon ${ }^{\mathrm{a}}$.

\begin{tabular}{|c|c|}
\hline Term & Meaning \\
\hline Alternagyre & A gyrosystem whose gyrapex is not triquantal \\
\hline Dextragyre & A right-handed gyre or gyromodel \\
\hline Focagyre & A gyre that is the focal point of analysis or discussion \\
\hline Gyradaptor & The gyre singularity - a quantum - that exerts all forces on the gyrosystem \\
\hline Gyrapex & The relativistically high potential, excited, unstable, learning state of a particle \\
\hline Gyraxiom & A fact, condition, principle, or rule that constrains and defines the theoretical framework \\
\hline Gyre & The spacetime shape or path of a particle or group of particles; a quantum \\
\hline Gyrequation & Shorthand notation for analysis, discussion, and understanding gyromodels \\
\hline Gyrobase & The relativistically low potential, ground, stable, memory state of a particle \\
\hline Gyrognosis & The thermodynamically demanding process of learning and integrating IEM \\
\hline Gyrolink & The mIEM particle that links two gyromodules in a gyronexus \\
\hline Gyromnemesis & The thermodynamically conserving process of remembering and recovering IEM \\
\hline Gyromodel & The core model undergirding the theoretical framework \\
\hline Gyromodule & A dIEM particle in a gyronexus \\
\hline Gyronexus & A polymer of dIEM particles linked by mIEM particles \\
\hline Gyrostate & The potential and/or kinetic state that a particle occupies in its gyratory path \\
\hline Gyrosystem & A gyromodel with specific IEM composition, organization, and purpose \\
\hline IEM $^{b}$ & Information, energy, and/or matter \\
\hline Levoragyre & A left-handed gyre or gyromodel \\
\hline Majorgyre & A gyrosystem whose gyrapex is triquantal \\
\hline Matrioshkagyre & A model that demonstrates how gyres organize in nested sets \\
\hline Ohiogyre & Higher-order organization in which a gyre gyrates around another gyre \\
\hline Particle & A discrete, finite, empirically definable unit of IEM \\
\hline Quantal & Of or relating to the quantum; tri-, di-, uni- and aquantal gyrostates found in majorgyres \\
\hline Quantum & A capacious, potentially infinite, uncertain unit of IEM; a gyre \\
\hline Subgyre & The gyre that subsumed by the focagyre \\
\hline Supragyre & The gyre that subsumes the focagyre \\
\hline Trimergence & Evolutionary emergence of a triquantal IEM \\
\hline \multicolumn{2}{|l|}{ Prefixes $^{c}$} \\
\hline Amino & Of or relating to sulfur compounds (particles), amino acids, polypeptides \\
\hline Carbo & Of or relating to carbon particles, carbohydrates, hydrocarbons \\
\hline Cellulo & Of or relating to cells, archaebacteria, eubacteria, eukaryotes \\
\hline Electro & Of or relating to visible matter particles, chemical elements, planetary cores \\
\hline Geno & Of or relating to genes, DNA, chromosomes, genomes \\
\hline Oxy & Of or relating to oxygen particles, water, oceans, lunar cores \\
\hline Phospho & Of or relating to phosphate particles, phospholipids, phosphate signaling \\
\hline Ribo & Of or relating to nitrogen particles, nitrogenous bases, RNA \\
\hline \multicolumn{2}{|l|}{ Suffixes $^{c}$} \\
\hline -cycle & The spacetime period to complete a regular series of events in the same order \\
\hline- gyre & $\begin{array}{l}\text { Having the quality of a vortex; characterized by cyclical, oscillatory, and unpredictable } \\
\text { motion; attractorepulsive, expansocontractive, and creatodestructive }\end{array}$ \\
\hline -gnose & Characterized by learning or by IEM consideration and integration \\
\hline -helix & Having a three-dimensional twisting, winding shape like that of a spiral staircase \\
\hline- matrix & Having a three-dimensional networked, latticed shape like graphene or an ice crystal \\
\hline- mneme & Characterized by memory or by IEM storage and retrieval \\
\hline
\end{tabular}


Table 1. Cont.

\begin{tabular}{cl}
\hline $\begin{array}{c}\text { Term } \\
\text { Suffixes } \\
\text { c }\end{array}$ & Meaning \\
-nexus & Being connected or linked in a series \\
-on & Having the quality of a quantum; a particle or an amalgam of such particles \\
-sphere & Having orb-like features and hyperbolic geometry \\
\hline
\end{tabular}

${ }^{\text {a }}$ This lexicon is presented alphabetically. In several circumstances, this ordering of words causes definitional cascading - that is, reading of word 1 uncovers an undefined word 2; reading the definition of word 2 reveals undefined word 3; the definition of word 3 provides an ultimate explanation and a meaningful backdrop for understanding words 1 and 2. ${ }^{\mathrm{b}}$ The gyromodel has defining IEM (dIEM) and modifying IEM (mIEM) particles. ${ }^{c}$ Each prefix is combined with each and every suffix to expand the lexicon of the theoretical framework. This neologistical appending reveals the commonality between, within, and among the distinct gyrosystems.

\section{Model}

Throughout history, scholars have used the gyre in their models. For example, in ancient Greece, Democritus posited vortex motion to be a law of nature. In the $16^{\text {th }}$ century, Copernicus modeled planets gyrating around a stellar singularity and Descartes proposed his vortex theory for planetary motion in the $17^{\text {th }}$ century. The $19^{\text {th }}$ century found Helmholtz rediscovering the Democritean law and Lord Kelvin and Maxwell using the gyre as the basis of different electromagnetic theories. In the early $20^{\text {th }}$ century, Bostick used the gyre in his spiraling helicon fiber model and Thomson proposed that atoms were vortex rings. Many others have promulgated the gyre as core model of nature.

Perhaps one reason for their theoretical appeal is that gyres are detectable throughout the cosmic and tellurian realms. Astronomically, galaxies, solar systems, comets, and lunar bodies gyrate. Atmospherically, tornadoes, hurricanes, eddies, and vortex streets are all gyres. Oceanographically, there are seven major gyres. Molecularly, numerous nucleic acid and protein structures-DNA double helix, RNA hairpins, pseudoknots, $\alpha$-helices, coiled coils, and $\beta$-propellers - all gyrate. Cellularly and organismally, shells, horns, antennae, flagellae, and the cochlea all carry a spiral imprint. Given its theoretical pedigree, empirical ubiquity, and dynamic character, the gyre appears, a posteriori, to be a prime candidate for a core model of natural systems.

\subsection{Gyre Facts}

There are numerous facts that characterize all gyres [25-28]. These facts-introduced here for propaedeutic purposes - demonstrate that the gyre is protean. For this presentation, I have separated these facts into four broad, overlapping categories and subsections: gyre structure, gyre qualities, gyre thermodynamics, and gyre forces. I conclude this section with a brief summary regarding the gyre and its relevance to theoretical pursuits.

\subsubsection{Gyre Structure}

A visual examination of the gyre reveals a remarkably plastic geometric form. That is, gyres manifest particular shapes and patterns of a non-Euclidean form. Viewed transversely, many gyres are elongated, helicoid, conical, or funnel-shaped, with a tapered bottom that ends in a point or singularity 
and have a wide aperture at their top. Other gyres are cylindrical, catenoid, flattened, or disc-like. When viewed head on, both the singularity and aperture frequently appear as perfect circles, like in a galactic center or the eye of a hurricane. Measurements from the singularity of a natural gyre to its circumferential aperture show exponential growth whereas the converse shows exponential decay. Any gyre is fractal because of its self-similarity, fine structure, and simple and recursive nature.

The gyre singularity is defined here as the central position around which energy and matter (discussed further in 2.3.1) revolve. The singularity is also the point of highest energy and matter density and potency in the gyre. Suggestive of the applicability of the gyre to modeling nature, the singularity concept is found both in astrophysics [29,30] and in life sciences [31]. Gyres are also symmetrical: they have organizational or compositional reflectivity, identity, or similarity around a radial axis that bisects the singularity. This symmetry is detectable in spiral galaxies, chemicals like heme, and macromolecules structures like the centrosome.

Gyres are chiral, i.e., have handedness. When viewed head on, a left-handed gyre rotates clockwise; a right-handed gyre rotates counter-clockwise. The paradox of chirality is that a left-handed gyre, when inverted $180^{\circ}$ and viewed anew, is a right-handed gyre. This paradox is at the core of the problem of life. Indeed, homochirality - exclusive use of one chiral form or the other-is observed throughout life, where sugars are dextral (D), amino acids in polypeptides are levoral (L) and nucleotides in nucleic acids are D form [32]. With this paradox in mind, the core, generic gyromodel can be viewed as either left-handed (levoragyre; (Figure 1a $(i)$ ) or right-handed (dextragyre; (Figure 1a (ii)).

\subsubsection{Gyre Qualities}

There are several characteristics of a gyre that make it theoretically appealing. Most notably, gyres are organic, that is, they have qualities identical to those found in living systems: they adapt their shape, size, position, rate, strength, and direction. Furthermore, gyres follow a life cycle of emergence (birth), development (aging), and dissolution (death). Gyres spontaneously self-organize when the pressure, temperature, energy, and matter conditions are appropriate. Foreshadowing gyromodel application, scientists have proposed that the universe, matter, molecules, cells, and ecosystems, among other aspects of nature, are self-organizing [33-36]. Given gyre spontaneity, the precise spatiotemporal coordinates of gyre emergence or trajectory are unpredictable. Likewise, accurately predicting gyre strength and composition is beyond current scientific techniques.

This unpredictability is found in nonlinear equations: gyres do not operate in a sequential or deterministic manner and therefore do not permit simple mathematical depiction. Restated, the versatile gyre does not avail itself to the predictive power of mathematics. As an aside, it is worth mentioning that a complete and consistent mathematical model of the universe is thought impossible due to Gödel's incompleteness [37,38]. The vicissitudinous gyre, though non-mathematical, epitomizes nature. 
Figure 1. Core theoretical concepts. (a) Gyromodel chirality. (i) Transverse view of a left-handed gyre (levoragyre). (ii) Transverse view of a right-handed gyre (dextragyre). The first and second half-turns of the gyres are depicted as bent arrows. White, gyre interior; grey, gyre exterior. (b) Archetypal gyromodel. This gyromodel-supplemented with symbolic variables - is an exemplar for understanding IEM emergence, adaptation, movement, and evolution in the natural world. The bold straight arrows represent IEM directionality. The first bold arrow, from the gyrapex $\left(\mathrm{X}^{\cdots *}\right)$ to the gyradaptor $(\odot)$, represents mIEM particle $(\bullet)$ attraction (absorption) to the singularity, causing the diquantal dIEM $\left(\mathrm{X}^{*}\right)$ to cycle to the gyrobase. The second bold arrow, from the gyradaptor to the gyrobase, represents the mIEM particle repelled (emitted) from the singularity, ultimately causing the diquantal dIEM to cycle to the gyrapex, restoring the triquantal dIEM (next cycle not shown here). The gyromodel thus depicts an open thermodynamic system. (c) Majorgyres. Majorgyres are the three main gyromodels at the core of each gyrosystem in the theoretical framework: $(i)$ primary $\left(1^{\circ}\right)$ majorgyre; $(i i)$ secondary $\left(2^{\circ}\right)$ majorgyre; and (iii) tertiary $\left(3^{\circ}\right)$ majorgyre. Note how the gyrapex is shared by all three majorgyres. (d) Gyre-quantum equivalence and Matrioshkagyres. Left-side equations. (i) The gyre - modeling the cycling $\bullet$ on/in and off/out of $\mathrm{X}$ due to the attractorepulsive quantum $\odot$ - is the compressed into $\mathrm{X}$, a quantum. (ii) $\mathrm{X}$, in turn, is the gyradaptive force responsible for cycling $\mathrm{X}$ on/in and off/out of $\mathrm{Y}$. Right-side equations. ( $i$ ) The $\odot$ is a dextral subgyre (dextrasubgyre) within the levorafocagyre. (ii) The levorafocagyre, in turn, is antichiral to the dextrasupragyre. $\mathrm{X}$ and $\mathrm{Y}$ ) are thus both antichiral Matrioshkagyres.

$\mathbf{a}_{(i)}$

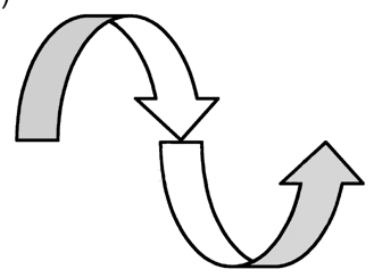

(ii)
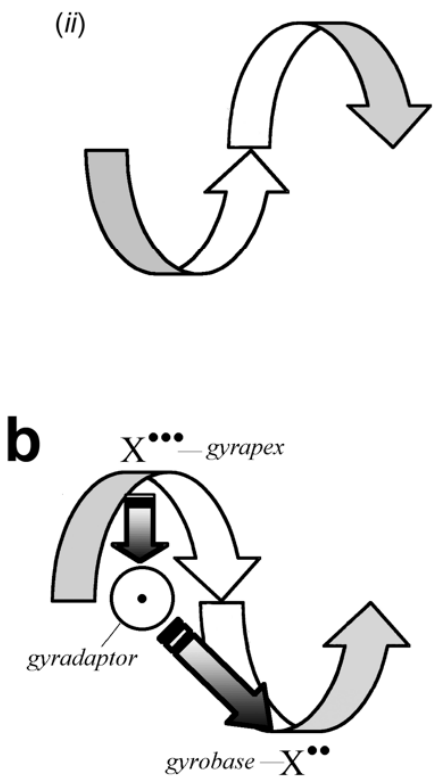

$\mathbf{C}_{(i)}$

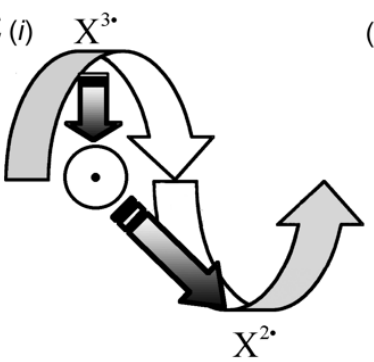

$\mathbf{d}_{(i)}$

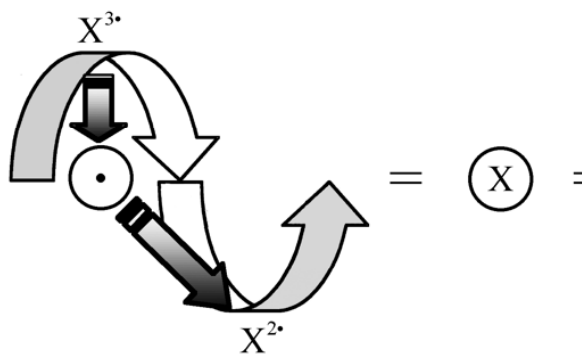

(ii)
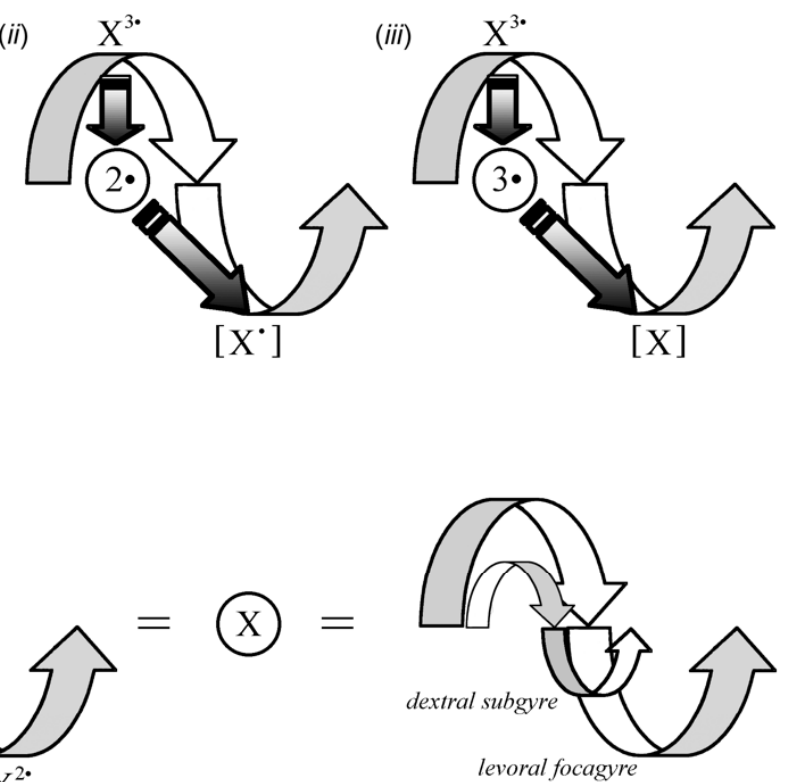

levoral focagyre
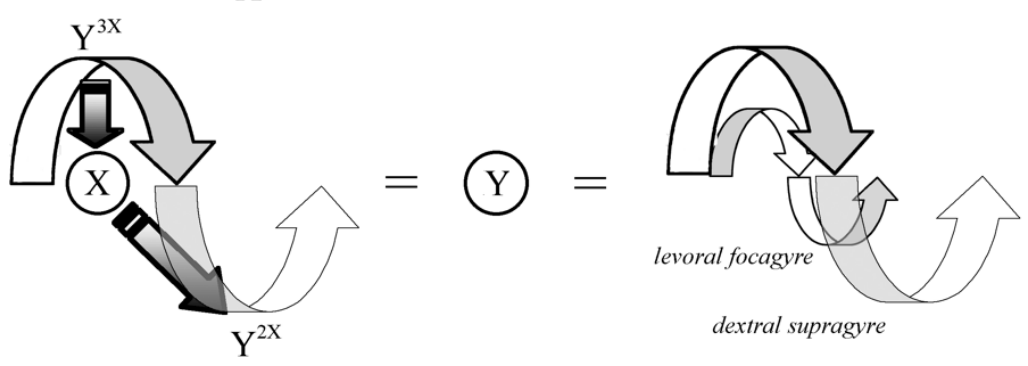


\subsubsection{Gyre Thermodynamics}

Gyres are open thermodynamic entities that require energy and matter mobilization to establish and maintain themselves. Being open systems, gyres import energy and matter from their surroundings into themselves, ebb and flow energy and matter within themselves, and dissipate energy and matter from themselves into their surroundings. Reducing or increasing amounts of energy and matter elicits gyre contraction or expansion, respectively. When efflux or influx is acute, extreme, or unsustainable, a gyre collapses. A gyre staves off collapse through autoregulation: a gyre feeds into itself, regulating its own rotational rate, size, composition, motion, and trajectory. Gyre autoregulation is spatiotemporally internal and/or external, proximal and/or distal, negative and/or positive. Consistent with its autoregulatory bent, a gyre maintains homeostasis — internal responsiveness and balance — by oscillating material around its singularity, a consequence of alternating between extreme countervailing forces within itself (2.1.4). Previewing the application of the gyromodel to life, the cell is an open thermodynamic entity that has numerous, discrete layers of autoregulation [39-41].

When considering the directionality of ebb and flow of a gyre, a careful examination reveals thatin the absence of physical blockage or inhibition - energy and matter at a spacetime point has potential to move omnidirectionally from that point. When this potential is actualized for an excessively large number of matter particles, the geometric form created is called a hypersphere; this shape is compatible with ideas regarding the thermodynamic expansion of the universe $[42,43]$. Still, in nature, there is manifest directionality, such as that observed in the $\mathrm{N}$ - to C-terminal orientation of the protein chain or 5' to 3' orientation of nucleotide polymers. Though gyromodels are depicted as having a left-to-right vectorization (Figure 1a), this is simply a two-dimensional restriction of the artistic approach. From this two-dimensional perspective, one revolution of a gyre is seen as a circle or oval. A circle, when viewed in three dimensions, is a cycle. Closing this circle of thought: a cycle viewed in the context of time, or four dimensions, looks like a rotating spiral, helix, or gyre. Foreshadowing, any cycle that exists in nature - in physical, chemical, or biological systems - may be viewed as a gyre.

\subsubsection{Gyre Forces}

All natural gyres harbor two countervailing forces: attraction and repulsion. Paradoxically, the gyre singularity both attracts and repels energy and matter and thus is "attractorepulsive." These unified yet contradictorily dual (diune) forces exert paradoxical effects. For example, individually, the attractive and repulsive forces can elicit both aggregation and dis-aggregation of energy and matter; these creative and destructive ("creatodestructive") effects are relative to the singularity. Moreover, a gyre can undergo expansion or contraction ("expansocontractive") as a consequence of energy and matter influx or efflux. Taken together, these countervailing forces make for a matrix of diune or multiple phenomena (e.g., "attractocontractive," "repulsocreative," "expansodestructive," and so on). Gyre forces occur both within an individual gyre and also between and among gyres. For example, two transverse gyres exhibit constructive interference when synchiral (same chirality) and destructive interference when antichiral (opposing chirality). Alternatively, the fine-tuning and balancing of two contradictory forces results in neutrality, immutability, and immobility-identifiable characteristics of physical systems. I detail these forces as they relate to the gyromodel in 2.4.6. 


\subsubsection{Gyre Summary}

The prior sections demonstrate that the gyre is a basic and concrete model of broad applicability, a profound heuristic, and an unchanging form that changes. Further, the gyre evidently fulfills many of the modeling requirements of complexity, emergence, chaos, systems, information, and evolutionary theory [44-51]. As such, the burden of proof for the gyre as the core model of nature is heavy; this manuscript represents the deposition of empirical testimony in theoretical court.

\subsection{Gyromodel Caveats}

Life is complex and perplexing. It should come as no surprise that modeling life is a complicated procedure. Likewise, explaining a theory of life is an arduous task. Thus, prior to proceeding, I issue several warnings regarding the model and theory.

The gyromodel is incommensurable with prior and existing theories. Thus, the reader must judge this theory by two criteria: the principle of parsimony, or Ockham's Razor [52] - the scientific principle dictating that things behave or are connected in the simplest and most economical fashion-and the ability to explain the available scientific data. Another challenge is discovered in the lexicon, where I have redefined established terms and created and applied $~ 100$ new words to identify, explain, and interconnect distinct aspects of the theory (Table 1). Creating a new vocabulary yields, on the one hand, a single, tight system to unify multiple disparate scientific languages. On the other hand, simultaneously supplanting the vernaculars of physics, chemistry, and biology may cause a high degree of frustration. Together, the foreign symbolism, semantics, and lexicon make comprehending the gyromodel difficult. As more is different [53], one must think differently to interpret more. Finally, this theory challenges long-held assumptions, guiding philosophies, ad hoc models, cherished paradigms, ossified boundaries, and, quite regrettably, patience.

These warnings represent a full and sincere disclosure of the difficulties in effectively presenting my model and theory and of convincing the reader of its scientific merit. I also mean to emphasize, up front, that this manuscript is dense.

\subsection{Gyromodel Organization}

I now begin the technical overview of the gyromodel in earnest. This section is presented in four subsections. The first defines information, energy, and matter and foreshadows how these three elements integrate throughout the theory. The second part establishes a gyromodel-specific relationship between a physical "particle" and a "quantum." The third part deals with two specific states that are modeled onto the gyre. The fourth and final part introduces three major gyromodel forms and applies a familiar notation to explore their explanatory utility. By laying out the core template and its rules in entirety prior to data fitting, I ensure that everything that is not prohibited is required.

\subsubsection{Information, Energy, and Matter}

The three main components that are represented by, stored in, and mobilized within, between, and among gyres are information, energy, and/or matter (IEM): Information is theoretically defined as something that conveys and harbors meaning; energy is something that is used to perform work; and 
matter is something that occupies space and has mass [54]. There are several points regarding IEM that require development. For instance, an object or a system can possess different types of energy, including but not limited to potential, kinetic, electromagnetic, chemical, and heat. Life is replete with examples of these different types: endo- and exothermic (energy input and energy output, respectively) biophysical processes, biochemical pathways, biogeochemical cycles, and inorganic chemical reactions [55-57]. Moreover, energy and matter are interconvertible based upon their mathematical equivalence $\left(\mathrm{E}=\mathrm{mc}^{2}\right)$ : energy can be transformed into matter and mass into energy. Information, in turn, is the distinct patterns or organizations of energy and matter, with these patterns detectable by observation and quale [58]. Even though certain modeled objects or systems could be considered as largely one of these three components, I use the acronym IEM throughout the text to denote the composite nature and interrelatedness of information, energy, and matter.

I integrate IEM into the gyromodel as symbols. These symbols are variables, representing one or more gyre components or set(s) of components. Although these symbols (components) are written adjacent to the gyre, they are the gyre itself. Throughout this exposition, I highlight important notations by offsetting them from the text.

\subsubsection{Particle and Quantum}

The generic gyromodel is decorated with several symbols (Figure 1b). For example, $\odot$ denotes the gyre singularity. For the compound symbols $\mathrm{X}^{\cdots \cdot \boldsymbol{*}}$ and $\mathrm{X}^{*}, \mathrm{X}$ is called the defining IEM $(\boldsymbol{d I E M})$ and $\bullet$ is modifying IEM (mIEM) of the gyre. Whereas the dIEM typifies a gyre system (gyrosystem) - there are several unique systems that require modeling - the mIEM changes the quality, content, or application of the dIEM. Any single (X or $\bullet$ ) or compound $\left(\mathrm{X}^{\cdots}\right.$ or $\left.\mathrm{X}^{* *}\right)$ component is called a particle. For theoretical thrift, $\mathrm{X}^{\cdots}$ or $\mathrm{X}^{*}$ represents either one particle or many particles of similar composition.

The singularity, $\odot$, models an IEM packet called a quantum. The quantum exerts an adaptive force on the gyrosystem (and thus is termed the gyradaptor), modulating the thermodynamic and spatiotemporal properties of particles. The quantum captures the infinite IEM potential:

$$
\bullet+\odot=\odot \text { (i.e., any number }+\infty=\infty \text { ) }
$$

In the gyromodel, there is an inverse conceptual relationship between a particle and a quantum: Though the quantum typically represents all germane IEM, it can represent one, this being the particle; though a particle typically represents one germane IEM, it can represent all, this being the quantum.

\subsubsection{Gyre States}

The generic gyromodel appears to show two particles, one occupying each gyre half-turn (Figure 1b). However, this is not two dIEM particles, but rather one dIEM particle that gyrates from one distinct, extreme spacetime state to another (gyrostates). The excited state $\left(\mathrm{X}^{\cdots *}\right)$ is the apex of the gyre, the gyrapex. The ground state $\left(\mathrm{X}^{*}\right)$ is the base of the gyre, the gyrobase. dIEM oscillation between gyrostates occurs via particle exchange to and from the gyradaptor. The mIEM particle, by comparison, can exist in only one of three spacetime coordinates: the gyrapex, gyrobase, or gyradaptor. Given gyrostate oscillation, the gyromodel accords with energetic coupling such as that seen in biometabolism. 


\subsubsection{Majorgyres}

Gyromodels with a triquantal (where $\bullet^{\bullet}=3^{\bullet}$ ) gyrapex and either a di- $\left(\bullet=2^{\bullet}\right)$, uni- $(\bullet)$, or aquantal gyrobase define the three majorgyres - the major gyromodels of the theoretical framework (Figure 1c). The gyrapex and primary majorgyre (1 ${ }^{\circ}$; Figure 1c $(i)$ ) have already been discussed as in the context of the generic gyromodel $(2.3 .2 ; 2.3 .3$; Figure $1 \mathrm{~b})$.

For the secondary majorgyre $\left(2^{\circ}\right.$; Figure $\left.1 \mathrm{c}(\mathrm{ii})\right)$, the gyradaptor is $2 \odot$, a diquantum, and the gyrobase, $\left[\mathrm{X}^{*}\right]$, where [ ] represents a unit that has the potential to multimerize, is:

$$
\mathrm{X}^{\bullet} \text { (monomer), } \mathrm{X}^{*} \mathrm{X}^{\bullet} \text { (dimer), } \mathrm{X}^{*} \mathrm{X}^{*} \mathrm{X}^{*} \text { (trimer), and/or } \mathrm{X}^{*} \mathrm{X}^{*} \mathrm{X}^{*} \mathrm{X}^{*} \mathrm{X}^{*} \mathrm{X}^{*} \mathrm{X}^{\bullet} \ldots \text { (polymers) }
$$

Note that the mIEM (•) "links" the dIEM (X) in the polymer; I expand upon the linkage concept below (2.4.3). In the tertiary majorgyre ( $3^{\circ}$; Figure $1 \mathrm{c}(\mathrm{iii})$ ), the gyradaptor is $3 \odot$, a triquantum, and the gyrobase, $[\mathrm{X}]$, is:

\section{$\mathrm{X}, \mathrm{XX}, \mathrm{XXX}$, and/or XXXXXX...}

Here, an IEM inherent to $\mathrm{X}$ itself is the quantal "link" (not shown to maintain modeling consistency). When $\left[\mathrm{X}^{*}\right]$ and $[\mathrm{X}]$ are two or more units, they are a particle nexus termed a gyronexus.

An accessible way of presenting these majorgyres is by reaction equation shorthand (gyrequations):

$$
\begin{gathered}
\text { Primary majorgyre: } X^{3 \bullet} \leftrightarrows X^{2 \bullet}+\odot \\
\text { Secondary majorgyre: } X^{3 \bullet} \leftrightarrows\left[X^{*}\right]+2 \odot \\
\text { Tertiary majorgyre: } X^{3 \bullet} \leftrightarrows[X]+3 \odot
\end{gathered}
$$

There are additional features of the gyromodel that can be elaborated using gyrequations. A majorgyre can be multiplicative, for example:

$$
\left(\mathrm{X}^{3 \bullet} \leftrightarrows \mathrm{X}^{2 \cdot}+\odot\right)_{\mathrm{n}}
$$

where $\mathrm{n}=$ any positive integer. These multiples, in turn, can undergo division or fractalization. Further, using this notation, majorgyres can be balanced like chemical reactions. Since $\mathrm{X}^{3 \cdot}$ is found in both primary and secondary majorgyres, these can be rewritten as:

$$
\mathrm{X}^{2 \cdot}+\odot \leftrightarrows \mathrm{X}^{3 \cdot} \leftrightarrows\left[\mathrm{X}^{*}\right]+2 \odot
$$

Removing the $\mathrm{X}^{3 \cdot}$ intermediate, thus compressing the gyrequation, reveals:

$$
\mathrm{X}^{2 \cdot}+\odot \leftrightarrows\left[\mathrm{X}^{\bullet}\right]+2 \odot
$$

And balancing the gyrequation by subtracting out $\odot$ from both sides gives:

$$
\mathrm{X}^{2 \cdot} \leftrightarrows\left[\mathrm{X}^{\bullet}\right]+\odot
$$

another pair of particle gyrostates; this represents one of many alternative gyre forms (alternagyres).

While a particle can be described by gyrequations in practice, it must be considered as part of larger gyre within which it resides in theory. By extension, a gyre must be considered in the context of its gyrosystem within which it exists. Despite their complexity, gyrosystems that share IEM can be multiplied, divided, added, or subtracted, allowing modeling of multi-component systems with gyrequations. Although a gyrequation excludes the vectorial and adaptive nature of IEM flow, it is a 
compact and tractable notation. Further, the gyrequation reminds of chemical equations that symbolically represent chemical reactions. Based upon this familiar and standardized format, I use gyrequations to substantiate and extend upon the gyromodels.

\subsection{Gyromodel Fundamentals}

The foremost purpose of this subsection is to introduce several fundamental features of the gyromodel. A secondary purpose is to give certain words - associated with nebulous, misunderstood, or complex concepts or phenomena-a model-specific meaning. I have organized this subsection into six parts. In the first, I explain the relationship between a gyre and a quantum. In the second, I discuss how the gyromodel treats complementary wave and particle forms. Third, I expand upon the quantal "link." Fourth, I model learning and memory onto the two gyrostates. Fifth, I introduce the concept of gyrosystem relativism. Finally, I close with a passage that explores gyromodel dynamics.

\subsubsection{Gyre-Quantum Equality}

In the gyromodel, a gyre is equivalent to a quantum (Figure $1 \mathrm{~d}(i)$, left side equations):

$$
\left(\mathrm{X}^{3 \bullet} \leftrightarrows \mathrm{X}^{2 \bullet}+\odot\right)=\mathrm{X}
$$

As shown, $X$ captures the full range of potentialities for the primary majorgyre. Importantly, however, $\mathrm{X}$ is variable, representing any majorgyre or alternagyre. Given gyre-quantum equivalence, then, $\mathrm{X}$ models a gyradaptor that cycles mIEM particles $(\mathrm{X})$ through dIEM particles $(\mathrm{Y})$ of a supervenient gyre (Figure 1d (ii), left side equations), which is itself a quantum, (Y).

For orientation, the gyre/quantum that is the focal point of analysis is the focagyre (focaquantum); the gyre/quantum subsumed by the focagyre is called a subgyre (subquantum); and that which subsumes the focagyre is called a supragyre (supraquantum). Thus, $\odot$ is a subgyre, $X$ is a focagyre, and (Y) a supragyre. IEM thermodynamics are unidirectionally simplified as follows:

$$
\rightarrow \odot \rightarrow \mathrm{X} \rightarrow \mathrm{Y} \rightarrow
$$

The arrows that flank the modeled gyrosystems depict the excluded intrinsic and extrinsic gyres; feedforward and feedback are also excluded.

Because gyre-quantum equality may elicit cognitive dissonance, it is useful to reify this concept by replacing the quantal form with the gyre form (Figures $1 \mathrm{~d}(i)$ and (ii), right side equations): $\odot$ becomes a dextral subgyre within $\mathrm{X}$, a levoral focagyre that is within $\mathrm{Y}$, the dextral supragyre. As this nested antichiral gyre organization is similar to that found in Matrioshka dolls, these models are called Matrioshkagyres. The countervailing forces exerted by antichiral Matrioshkagyres impart gyroscopic stability. Matrioshkagyres can also be synchiral. However, the greater the IEM flow rate, potency, and amount, the higher the probability that a synchiral Matrioshkagyre will become imbalanced, torsionally stressed, and collapse. Thus, oscillating chirality of gyres is essential for gyre maintenance and propagation.

Modeling the Matrioshkagyre in a gyrequation, the supragyre is

$$
\mathrm{Y}^{3 \mathrm{X}} \leftrightarrows \mathrm{Y}^{2 \mathrm{X}}+\mathrm{X}
$$


where, given gyre-quantum equivalence,

$$
\mathrm{X}=\mathrm{X}^{3 \bullet} \leftrightarrows \mathrm{X}^{2 \bullet}+\odot
$$

then:

$$
\mathrm{Y}^{3 \mathrm{X}} \leftrightarrows \mathrm{Y}^{2 \mathrm{X}}+\left(\mathrm{X}^{3 \cdot} \leftrightarrows \mathrm{X}^{2 \cdot}+\odot\right)
$$

For the sake of this propaedeutic, let

$$
\odot=\cdot^{3 Z} \leftrightarrows \cdot 2 Z+(Z)
$$

then, substituting again, I have:

$$
\mathrm{Y}^{3 \mathrm{X}} \leftrightarrows \mathrm{Y}^{2 \mathrm{X}}+\left(\mathrm{X}^{3 \cdot} \leftrightarrows \mathrm{X}^{2 \cdot}+\left(\cdot \bullet^{3 \mathrm{Z}} \leftrightarrows \cdot 2 \mathrm{Z}+(\mathrm{Z})\right)\right)
$$

This schema captures the nested relationship among primary majorgyrosystems, the inherent variability of each, and how one cannot be studied independently without loss of IEM of another. Complexifying further, given the inverse quantum-particle relationship and gyre-quantum equivalence, each mIEM can model as a gyrosystem as well:

$$
\begin{aligned}
& \left.\mathrm{Y}^{3\left(\mathrm{X}^{3 \cdot} \leftrightarrows \mathrm{X}^{2 \cdot}+(\cdot 3 \mathrm{Z}\right.} \leftrightarrows \cdot 2 \mathrm{Z}+(2)\right) \leftrightarrows
\end{aligned}
$$

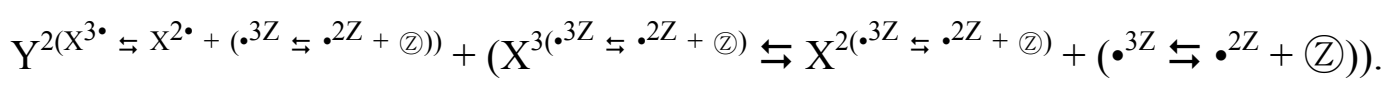

Because Matrioshkagyres and gyrosystems are continually adapting and can be any combinations of major- or alternagyres, the fractal depth of any gyrequation is infinite.

\subsubsection{Wave-Particle Unity}

The gyromodel clarifies how a quantum has both wave and particle qualities: as one particle oscillates between two extreme gyrostates, its gyratory path creates an undulating pattern that is detected as a wave. When many particles oscillate around the same or different singularities, they create constructive or destructive waveforms. When the gyromodel is considered as a gyre, it manifests classical wave characteristics: wavelength, amplitude, and frequency. When considered as a quantum, it exhibits particle characteristics: translational, rotational, and vibrational movement. The gyromodel thus accounts for particle spin.

\subsubsection{Gyronexus Links and Modules}

With gyre-quantum equivalence and quantum-particle relations disclosed, I am now able to expand upon the gyronexus "link" as was described for the secondary and tertiary majorgyres (2.3.4). Reviewing, a gyronexus is composed of two parts: the dIEM particle that is being linked (called here a gyromodule), and the mIEM particle that links (a gyrolink). When modeling the gyronexus in a secondary majorgyre, the gyrolink that is a subgyre mIEM has antichiral spin to the gyromodular dIEM. For the tertiary majorgyre, the gyrolink that is a sub 2 gyre (the gyre within the subgyre) mIEM has synchiral spin to the gyromodular dIEM.

In addition to envisioning the dIEM and mIEM as spinning particles, one must also think of higher-order rotations, or orbits, of one particle around another-i.e., a gyrolink spinning on its own axis while simultaneously orbiting an axially rotating gyromodule. Hence, a gyrolink is dynamic, 
not static. Building upon this dynamism, since every particle is attractorepulsive, one gyrolink particle can mobilize from one opposing gyromodular area to another in a toroidal or plectonemic spacetime path. Examples of polymers that exist in the natural world for which the gyronexus concept applies: oxygen atoms linked by hydrogen atom electrons as found in water; oxygen atoms from water link carbohydrates in polysaccharides; orthophosphate links nucleotides in an RNA chain; and amide groups link amino acids in a polypeptide.

\subsubsection{Gyromodel Learning and Memory}

In this section, I articulate how the dynamics and interchangeability of information, energy, and matter within a gyre relates to the retention of these things within the gyre itself. In other words, I establish strict non-cognitive meanings for learning and memory as they relate to the gyromodel. Learning is a continual, unstable, and energetically demanding affair. Gyre learning, or gyrognosis, is the process by which the gyradaptor repels the particle from the gyrobase to the gyrapex. This is vectorially modeled in the primary majorgyre as:

$$
\odot+\mathrm{X}^{\bullet \bullet} \rightarrow \mathrm{X}^{\cdots \bullet \bullet}
$$

where ${ }^{\circ}$ represents the learned IEM. Gyrognosis also involves the reorganization of the learned IEM in the gyrapex, for example,

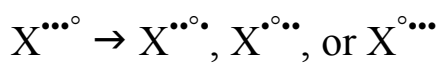

Memory, by comparison, is a relativistically stable and energetically conserving phenomenon. The process of storing IEM in gyre memory, or gyromnemesis, is modeled as:

$$
\mathrm{X}^{\cdots \cdot \bullet} \rightarrow \odot+\mathrm{X}^{\bullet^{\circ}}
$$

The ultimate state of gyromnemesis is the stably adapted particle or gyronexus in the gyrobase. A particle thus adapts through learning and memory by completing one full cycle-a revolutionaround the singularity. Taken together, gyrognosis defines IEM integration and assessment whereas gyromnemesis defines IEM storage and recovery. Finally, although a diquantal IEM ( $\mathrm{X}^{* *}$ ) undergoes gyrognosis as the gyrobase of a primary majorgyre, it undergoes gyromnemesis as the gyrapex of an alternagyre. Thus, gyre learning and memory are relative to the gyradaptive singularity.

\subsubsection{Gyromodel Relativity}

In considering the majorgyre frame, whereas the gyrapex of the three majorgyres is always the same, the gyrobase and gyradaptor of each is different (Figure 1c). These differences should be considered relativistically. For example, at least symbolically, the $1^{\circ}$ gyrobase has compositionally more IEM than does the $2^{\circ}$ gyrobase, and the $2^{\circ}$ gyrobase has more than the $3^{\circ}$ gyrobase. All other things being equal, the IEM in the gyrobases (di-, uni-, and aquantal states) of majorgyres could be written relativistically:

$$
1^{\circ}>2^{\circ}>3^{\circ}
$$


In comparison, all things being equal, the IEM in the gyradaptors of the majorgyres is, relativistically:

$$
3^{\circ}>2^{\circ}>1^{\circ}
$$

These three aspects of the gyromodel (or any other triad, i.e., triquantal, diquantal, and uniquantal forms) should be viewed as follows: (i) a high energy (exergic), unstable, excited form; (ii) an intermediate energy, quasi-stable, transition form; and (iii) a low energy, stable, ground form. Note the antiparallel relationship between gyrobases and gyradaptors, where the unstable gyrobase is accompanied by the stable gyradaptor, and vice versa. However, given that many disparate and dynamic IEM species need to be incorporated, gyromodel relativity is ever changing.

\subsubsection{Gyromodel Dynamics}

Here, I elaborate on gyromodel forces, directionality, chirality and collapse. As the Matrioshkagyre (Figure 1d) models, the singularity of a gyre is a gyre itself. In any given Matrioshkgyre, then, the attractive and repulsive forces of the supragyre are inherent, a consequence of the attraction and repulsion of the focagyre, which, in turn, are a consequence of the attraction and repulsion of the subgyre; and so on. So, then, while a specific force by or on a particle within a given gyromodelattraction, repulsion, expansion, contraction, creation, destruction-is albeit separable in principle, it needs to be considered in the fuller, nested context of other forces. Adumbrating an application of the gyromodel in biology, the catabolism (destruction, consumption) of glucose in glycolysis can be and is studied as a linear process and in isolation. However, this process is fundamentally dependent upon__and thus inseparable from - glucose anabolism (creation, production), both in the cell and during nonlinear evolutionary emergence.

In addition to forces within a gyromodel, forces are exerted between and among gyromodels. On this matter, gyromodel orientation and IEM composition impacts ebb and flow. When two parallel synchiral shared-IEM gyromodels (though similar, called here $\alpha$ and $\beta$ for distinguishability) juxtapose, the $\alpha$-singularity attracts $\beta$-dIEM and -mIEM particles and $\beta$-singularity attracts $\alpha$-dIEM and -mIEM particles, thereupon coalescing into a single, larger gyromodel. Such natural concrescence can be observed, for example, when two waterspouts merge or when two oil droplets fuse. By comparison, the singularities of two antiparallel synchiral shared-IEM gyrosystems attract one another to create a shared singularity and counter-mobilized IEM; this arrangement can be found in two antiparallel bar magnets (aligned side-by-side such that the $\mathrm{N}$ and $\mathrm{S}$ poles of one magnet appose, respectively, the $\mathrm{S}$ and $\mathrm{N}$ poles of the other) or in the DNA double helix. Repulsion between gyres-which models gyrosystem individuation - arises due to opposed directionality (head-to-head or tail-to-tail as opposed to head-to-tail) or of the inability to share IEM (i.e., gyromodels cycle or harbor distinct dIEM and mIEM). The repulsive (anti-attractive) effects of opposed directionality can be observed experimentally: when two $\mathrm{N}$ poles or two $\mathrm{S}$ poles of two bar magnets are apposed or when two 5' ends or two 3' ends of DNA oligomers are apposed.

As mentioned in 2.1.3, gyre collapse occurs by two extreme means: overcontraction or overexpansion. When, for a given gyre within a gyrosystem, a triquantal mIEM particle is attracted to the singularity, the dIEM particle loses the thermodynamic support of its gyradaptor (the gyrobase of 
the $3^{\circ}$ majorgyre; Figure 1c $($ iii $)$ ). Due to autoregulatory feedback within the gyrosystem, accelerative attraction of the singularity spreads (to all existing $1^{\circ}$ and $2^{\circ}$ majorgyres and alternagyres) and mIEM extraction collapses the gyrosystem into more exergic subgyres. Overexpansion-due to unrelenting influx of mIEM particles - forces the triquantal mIEM onto the dIEM (the gyrapex of all majorgyres), disallowing cycling between two states, thus freezing and ultimately collapsing the gyrosystem. An example of overcontraction in nature is desertification, where water collapse elicits diminished vegetation in an ecological system [59]. An example of overexpansion is in the life cycle of the slime mold Dictyostelium discoideum: the multicellular fruiting body state collapses, releasing single-celled spores [60]. Another example of overexpansion is wave collapse in physical systems [61].

\subsection{Gyromodel Axioms}

The forthcoming data placement onto the gyromodel complies with natural laws and ordering principles. In addition to using fundamental strictures, I codify a specific set of inviolable rules, conditions, and truths that guide and undergird the theoretical framework - thirteen major gyromodel axioms (gyraxioms) and several axiomatic corollaries. This section explains the application and relevance of these gyraxioms to the impending gyrosystems. For emphasis, the gyraxioms have been italicized.

Gyraxiom I $\left(G_{I}\right)$. A quantum is a gyre. This precept is based upon the equivalence of these entities as they are verbally defined (2.1 and 2.3.2) and symbolically depicted (Figure 1d).

$G_{I I}$. A gyrating particle is a wave. As gyromodeled (Figure 1b), the particle discloses the nature of its spacetime trajectory as an undulating waveform (2.4.2).

$G_{I I I}$. The quantum is either one particle or many particles. $G_{I I I-1}$. A particle has quantum potential. These two axioms, originally introduced in 2.3.2, reveal the flexibility of the gyromodel to incorporate the small and the large, the few and the many.

$G_{I V}$. A particle cannot be reduced from its gyre without IEM loss. A gyre is a unified, coherent entity. Any gyre aspect cannot be examined separately without decoherence and loss of contextualized information, energy, and/or matter. $G_{I V-1}$. A gyre cannot be reduced from its gyrosystem without IEM loss. As a gyrosystem is composed of and defined by numerous alterna- and majorgyres (i.e., quanta), gyre composition, structure, and function changes upon isolation from its gyrosystem (2.3.4).

$G_{V}$. A particle oscillates between excited and ground states but cannot simultaneously exist in more than one state in spacetime. A particle transits from one extreme gyrostate to another (Figures 1b,c, and $2 ; 2.3 .3$ ).

$G_{V I}$. A gyre oscillates between left and right chirality but cannot simultaneously exist as more than one chirality in spacetime. Relative to an observer, the gyrating trajectory of one particle or system can be either clockwise or counter-clockwise, but not both (Figure 1a; 2.1.1, 2.4.1).

$G_{V I I}$. Antichiral Matrioshkagyres are more homeostatic and stable than synchiral Matrioshkagyres. As introduced in 2.4.1, countervailing gyre chiralities of nested gyrosystems bestows stability on those systems; identical chiralities in nested systems induce runaway gyrosystem acceleration, torque, and disintegration. 
$G_{V I I I}$. A focagyre is thermodynamically dependent upon one or more of its subsumed gyres. $G_{V I I I-1}$. A focagyre is thermodynamically required for one or more of its supervenient gyres. Gyrosystem nesting depicts IEM flowing from within to without, from sub- to foca- to supragyre (2.4.1).

$G_{I X}$. A focagyre contains at least one novel, emergent IEM form distinct from its subgyre. The omnidirectional expansion and self-organized criticality of the subgyre yields a focagyre with similar organization yet dissimilar composition.

$G_{X}$. In a secondary majorgyre, the gyrolink of the gyronexus is the dIEM of the subgyre. The gyrolink of the gyronexus represents the subgyre itself $(2.3 .4,2.4 .3)$.

$G_{X I}$. In the tertiary majorgyre, the gyrolink of a gyronexus is the dIEM of the sub 2 gyre. $G_{X I-1 .}$ A tertiary majorgyre gyrolink, in coupling to other tertiary majorgyres, facilitates IEM flow between and among subsumed gyrosystems. These two gyraxioms disclose how long-range IEM thermodynamics and gyrosystem organization occurs $(2.3 .4,2.4 .3)$.

$G_{X I I}$. The IEM in primary and secondary majorgyres has subgyre chirality. In other words, dextral IEM oscillates in a levoral focagyre due to force exerted by dextral subgyre. Levoral IEM oscillates within a dextral focagyre due to force exerted by levoral subgyre (see Figure 1d). $G_{X I I-1}$. When countervailing forces of an antichiral Matrioshkagyre offset exactly, the focagyre IEM does not have chirality. This axiom provides a basis for a particle without spin or a neutral, illusorily immobile (non-gyrating) state. $G_{X I I-2}$. When considering $G_{X I}$, the IEM in the gyrobase of the tertiary majorgyre has sub ${ }_{2}$ gyre chirality. $G_{X I I-3}$. Given $G_{V}$ and $G_{V I}$, IEM higher-order organization and fractalization within a focagyre elicits chiral toggling. As Matrioshkagyres are more stable in an antichiral state, so too complexified IEM within a gyrosystem itself achieves intrinsic balance through countervailing nested chiralities (2.4.3).

$G_{X I I I}$. Subgyres are more exergic and less stable than focagyres. Relativistically, the subgyre IEM is of a higher quality of energy that is extractable for work. For example, the amount of energy that can be extracted to perform physical work from an electron (i.e., to transfer electricity) is greater as compared to the amount of energy extracted to perform physical work from a nucleotide triphosphate (i.e., to transfer an orthophosphate bond).

\section{Theory}

I have organized this part into eight subsections, each detailing a discrete, empirically defined system that is amenable to theoretical modeling: visible matter, water, organic matter, phosphomembrane, RNA, protein, DNA, and cell. This theoretical framework concomitantly depicts both the microcosmthe biology, chemistry, and physics of the existing living cell — and the macrocosm — the astrophysical and biogeophysichemical (geospheric, hydrospheric, atmospheric, biospheric) process underlying the evolution of life on Earth. Hence, subdividing this framework into separate parts defined by scale, by field, by topic, or by evolutionary spacetime is not scientifically appropriate for modeling life in toto. Given the manuscript format, a full treatment of alternative theories, models, hypotheses, and arguments is unrealistic. Nevertheless, each subsection overviews germane problems and theories, fits data using gyromodels and gyrequations, and concludes with a point regarding macroevolutionary transition from one quantal form to another. To enhance readability, each subsection is suborganized, with subject matter preceded by self-explanatory paragraphic title. 
Throughout this section, I point out four theoretical applications: ( $i$ ) to explain phenomena that have been enigmatic or misinterpreted; (ii) to model the organization of particles, atoms, molecules, and systems; (iii) to position the origin, emergence, and evolution of one thing relative to another; and (iv) to predict modes of operation. Each gyrosystem (Figure 2) is given a unique letter identifier and an etymologically obvious terminology (Table 2). Unless stated otherwise, a gyrosystem neologism refers to more than one or all majorgyres and alternagyres.

Figure 2. Gyromodels of the theoretical framework. (a) Gyromodels of leptonic metabolism. (i) $1^{\circ}$, (ii) $2^{\circ}$, and (iii) $3^{\circ}$ electrogyre; (iv) electron (e). This quantal form and all subsequent forms represent any of the majorgyres or alternagyres (not shown); $\gamma$, photon. (b) Gyromodels of oxychemical metabolism. (i) $1^{\circ}$, (ii) $2^{\circ}$, and (iii) $3^{\circ}$ oxygyre; (iv) oxyon (O). (c) Gyromodels of organochemical metabolism. (i) $1^{\circ}$, (ii) $2^{\circ}$, and (iii) $3^{\circ}$ carbogyre; (iv) carbyon (C). (d) Gyromodels of phosphochemical metabolism. (i) $1^{\circ}$, (ii) $2^{\circ}$, and (iii) $3^{\circ}$ phosphogyre; (iv) phosphon (P). (e) Gyromodels of ribonucleotide metabolism. (i) $1^{\circ}$, (ii) $2^{\circ}$, and (iii) $3^{\circ}$ ribogyre; (iv) ribon (R). (f) Gyromodels of amino acid metabolism. (i) $1^{\circ}$, (ii) $2^{\circ}$, and (iii) $3^{\circ}$ aminogyre; (iv) aminon (A). (g) Gyromodels of deoxynucleotide metabolism. (i) $1^{\circ}$, (ii) $2^{\circ}$, and (iii) $3^{\circ}$ genogyre; (iv) genon (D).

(h) Gyromodels of cellular metabolism. (i) Hapcellulogyre; (ii) dipcellulogyre; (iii) acellulogyre; (iv) cellulon (C). Note the repetitive yet chirally oscillating nature of gyrosystems. This figure complements Table 2.
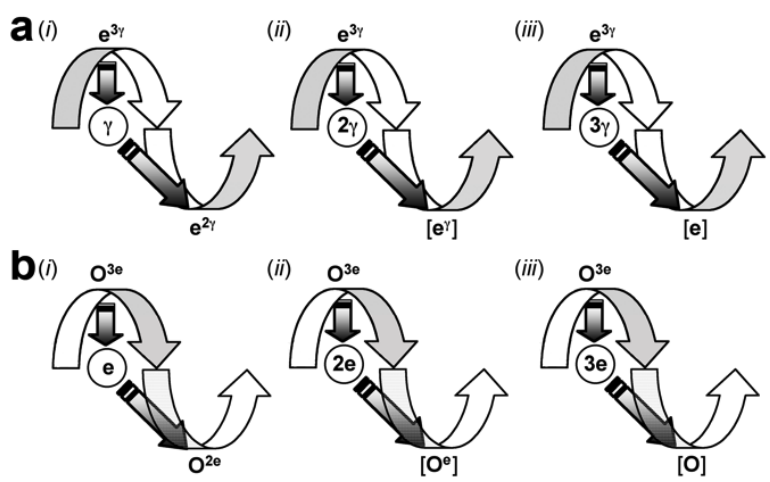

(iv)

(e)

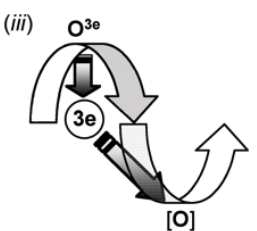

(iv)

(†)
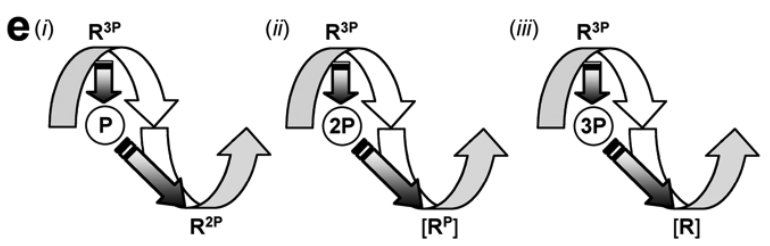

(iv)
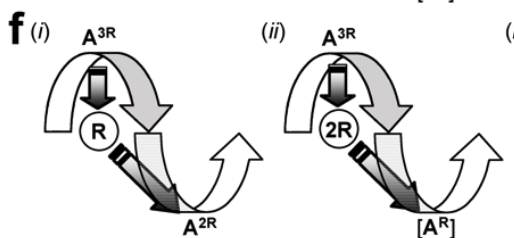

(iii) $\mathrm{A}^{3 \mathrm{R}} \quad$ (iv)
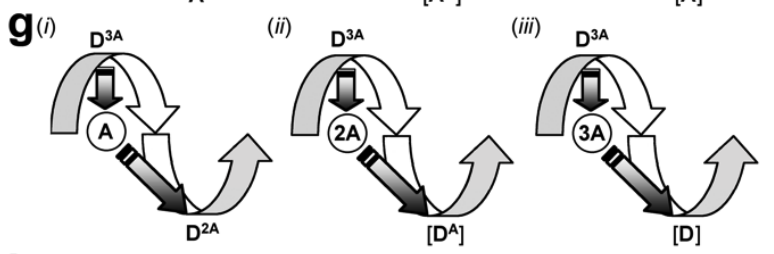

(iv)
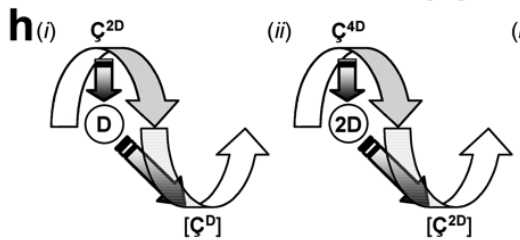

(iii) $\mathrm{C}^{4 \mathrm{D}} / \mathrm{Ç}^{2 \mathrm{D}}$

(iv)

There are two points to be made about referencing. First, in many cases, I use only one or two references to defend a position or to guide the reader. I regret that many seminal studies are not cited. Second, in the later subsections, I call attention to literature on the evolution and function of life as it relates to eubacteria, archaebacteria, and eukaryotes. However, I often cite only the most general and 
principal work on one kingdom or another and on one organism or another. Given these circumstances and if warranted, I urge the incisive and demanding reader to pursue deeper investigation of a particular field or topic.

Table 2. Gyrosystem organization ${ }^{a}$.

\begin{tabular}{|c|c|c|c|c|c|c|c|c|c|}
\hline \multirow[b]{2}{*}{ Gyre } & \multirow[b]{2}{*}{ Quantum } & \multirow[b]{2}{*}{ dIEM } & \multirow[b]{2}{*}{ mIEM } & \multirow[b]{2}{*}{ Gyradaptor } & \multicolumn{2}{|c|}{ Gyrostates $^{b}$} & \multicolumn{3}{|c|}{ Chirality $^{c}$} \\
\hline & & & & & Gyrapices & Gyrobases & Gyre & $1 \% 2^{\circ}$ & $3^{\circ \mathrm{e}}$ \\
\hline Electrogyre & Electron & $\mathrm{e}$ & $\gamma^{\mathrm{d}}$ & (7) & $\mathrm{e}^{3 \gamma}$ & $\mathrm{e}^{2 \gamma},\left[\mathrm{e}^{\gamma}\right],[\mathrm{e}]$ & $\mathrm{L}$ & $\mathrm{D}$ & $\mathrm{L}$ \\
\hline Oxygyre & Oxyon & $\mathrm{O}$ & $\mathrm{e}$ & (e) & $\mathrm{O}^{3 \mathrm{e}}$ & $\mathrm{O}^{2 \mathrm{e}},\left[\mathrm{O}^{\mathrm{e}}\right],[\mathrm{O}]$ & $\mathrm{D}$ & $\mathrm{L}$ & $\mathrm{D}$ \\
\hline Carbogyre & Carbyon & $\mathrm{C}$ & $\mathrm{O}$ & () & $\mathrm{C}^{30}$ & $\mathrm{C}^{2 \mathrm{O}},\left[\mathrm{C}^{\mathrm{O}}\right],[\mathrm{C}]$ & $\mathrm{L}$ & $\mathrm{D}$ & $\mathrm{L}$ \\
\hline Phosphogyre & Phosphon & $\mathrm{P}$ & $\mathrm{C}$ & (C) & $\mathrm{P}^{3 \mathrm{C}}$ & $\mathrm{P}^{2 \mathrm{C}},\left[\mathrm{P}^{\mathrm{C}}\right],[\mathrm{P}]$ & $\mathrm{D}$ & $\mathrm{L}$ & $\mathrm{D}$ \\
\hline Ribogyre & Ribon & $\mathrm{R}$ & $P$ & (P) & $\mathrm{R}^{3 \mathrm{P}}$ & $\mathrm{R}^{2 \mathrm{P}},\left[\mathrm{R}^{\mathrm{P}}\right],[\mathrm{R}]$ & $\mathrm{L}$ & $\mathrm{D}$ & $\mathrm{L}$ \\
\hline Aminogyre & Aminon & A & $\mathrm{R}$ & (B) & $A^{3 R}$ & $\mathrm{~A}^{2 \mathrm{R}},\left[\mathrm{A}^{\mathrm{R}}\right],[\mathrm{A}]$ & $\mathrm{D}$ & $\mathrm{L}$ & $\mathrm{D}$ \\
\hline Genogyre & Genon & $\mathrm{D}$ & A & (A) & $\mathrm{D}^{3 \mathrm{~A}}$ & $\mathrm{D}^{2 \mathrm{~A}},\left[\mathrm{D}^{\mathrm{A}}\right],[\mathrm{D}]$ & $\mathrm{L}$ & $\mathrm{D}$ & $\mathrm{L}$ \\
\hline Cellulogyre & Cellulon & Ç & $\mathrm{D}$ & (D) & $C^{4 \mathrm{D}}, C^{2 \mathrm{D}}$ & {$\left[C^{2 \mathrm{D}}\right],\left[C^{\mathrm{D}}\right],[\mathrm{C}]$} & $D^{f}$ & $L / D^{f}$ & $D / L^{f}$ \\
\hline
\end{tabular}

${ }^{\mathrm{a}}$ This table complements the gyromodels in Figure $2 .{ }^{\mathrm{b}}$ Gyrapices are the learning gyrostates; gyrobases are the memory gyrostates. The gyrostates for majorgyres are shown. ${ }^{c}$ Gyre and IEM exist cosmically in both chiralities but in life are almost exclusively in one chirality (see footnote 'e'). D, dextral; L, levoral. ${ }^{\mathrm{d}}$ The ' $\gamma$ ' models the photon. ${ }^{\mathrm{e}} 1^{\circ}$, primary majorgyre IEM; $2^{\circ}$, secondary majorgyre IEM; and $3^{\circ}$, tertiary majorgyre IEM. ${ }^{f}$ Several gyraxioms $-G_{\mathrm{VI}}, G_{\mathrm{VII}}, \mathrm{G}_{\mathrm{VIII}}, \mathrm{G}_{\mathrm{XII}}$, and $\mathrm{G}_{\mathrm{XIII}}$-clarify why the gyre and IEM chirality are "primarily" one form in cells as opposed to exclusively one form. As

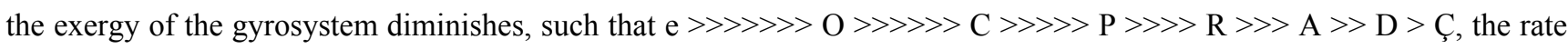
of IEM flow concomitantly diminishes. The relativistically reduced attractorepulsive effect of the genon on the cellulogyre (compared to earlier gyrosystem relationships) means that the subgyres have greater potential to impact cellulogyre form and function. Thus, while oscillating chirality $\left(\mathrm{G}_{\mathrm{VI}}\right.$ and $\left.\mathrm{G}_{\mathrm{XII}}\right)$ is retained in principle, theory fits the data in practice.

\subsection{Visible Matter}

I begin this theory with the modeling of visible matter, that is, spacetime particles that have mass and can be experimentally manipulated and visualized. I have chosen to start here because, in life, cellular respiration requires an electron transport chain [62], electron mobilization is fundamental to biophysical assembly and disassembly, biochemical reactions, and signal transduction [63], and, most notably, atomic and quantum models undergird the thinking and experimentation about all cellular molecules [64]. I now turn to modeling life from within to without, from the physics of the quantum to the biology of the cell.

There is overt discussion of a crisis in fundamental physics [65,66]. One reason for this harsh admission is the lack of a solution for the most protracted problem in modern physics: a model that unifies the atomic and cosmic realms [67]. From the early $20^{\text {th }}$ century, there have been two separate models for these two domains. Quantum mechanics (QM) is a mathematical model that describes the physical properties of visible matter [68]. General relativity (GR) is a mathematical theory that describes the universal attractive force, gravity [69]. A unified model of quantum gravity (QG) is expected to explain both the regular dynamics and properties of celestial bodies and all of the well-known quantum properties and enigmas such as spin, wave-particle duality, Heisenberg Uncertainty Principle, and the measurement problem [70]. Such a theoretical marriage would be expected to yield insight into the structure, function, and origin of leptons, chemical elements, planets, and solar systems. In this subsection, I introduce a gyrosystem called the electrogyre (Figure 2a) and, under the direction of natural laws and gyraxioms, place it onto physical data. 
Lepton. Microcosmically, the primary electrogyre (Figure 2a (i)) models the single free high-energy electron (e) found most commonly in the element hydrogen or any lepton (muon, tau, and neutrinos, each represented by the e symbol). Although there are many models of lepton substructure, the exact composition is unclear [71]. As modeled here, the lepton is a visible energy particle (a photon, $\gamma$ ) that step-decelerates below light speed due to the opposing, balancing forces and directionalities of the subgyre/subquantum $\left(\circledast ; \mathrm{G}_{\mathrm{VI}}, \mathrm{G}_{\mathrm{VII}}\right.$, and $\left.\mathrm{G}_{\mathrm{XIII}}\right)$. In other words, in reducing its vibrational, rotational, and translational rate, the photon particle literally manifests as the lepton particle.

The structure of the primary majorgyre requires a triquantal or diquantal set of photons (triphoton, $3 \gamma$, and diphoton, $2 \gamma$ ) to orbit the e particle, thus existing in either an excited ( $\mathrm{e}^{3 \gamma}$; the gyrapex) or ground $\left(\mathrm{e}^{2 \gamma}\right.$; the gyrobase) state in spacetime $\left(\mathrm{G}_{\mathrm{V}}\right)$. Oscillation between these two electrogyrostates occurs by virtue of the attractorepulsive, expansocontractive, and creatodestructive forces of the photonic singularity (\$) the gyradaptor).

To facilitate comprehension of the photon-as-singularity concept, I have presented it in four different ways in Figure 3a. The first fits the gyromodel to the evidence about the photon to the oscillating electron energy states (Figure 3a $(i)$ ), the second reveals the antichiral nature of and nesting of photons and electrons (Figure 3a (ii)), the third shows the known physical reaction (Figure 3a (iii)) and the fourth is the primary electrogyre as a gyrequation (Figure 3a (iv)). Looking ahead, I employ these four approaches for one exemplar of each other gyrosystem singularity.

Two gyraxioms help clarify the asymmetric and oscillating spin and chirality of leptons [72]. Specifically, $\infty$ is predicted to be a dextragyre that imparts a dextral spin on e $\left(G_{X I I}\right)$ whereas gyrosystemic balance occurs by the particle's trajectory being levoral $\left(\mathrm{G}_{\mathrm{VI}}\right)$. Together, these gyraxioms and this gyrosystem (Figure 2a) explain the chirality of atoms ([73]; Figure 4a). Recall that each symbol in the gyrequation represents one or many particles and that each gyrosystem and -equation can be compressed or expanded accordingly. On this matter, the electrogyre accommodates the three generations of leptons [74], where the proximity to the gyradaptive singularity dictates the stability and energy of the particle.

Higher-order Lepton Organization. The gyrobase of the secondary electrogyre (Figure 2a (ii)) represents a low-energy electron monomer (unpaired electron), dimer (lone pair, Cooper pair), trimer, or polymer (electronexus) in any non-hydrogen atomic orbital [75,76]:

$$
\left[\mathrm{e}^{\gamma}\right]_{\mathrm{n}}=\mathrm{e}^{\gamma}, \mathrm{e}^{\gamma} \mathrm{e}^{\gamma}, \mathrm{e}^{\gamma} \mathrm{e}^{\gamma} \mathrm{e}^{\gamma} \text {, and } \mathrm{e}^{\gamma} \mathrm{e}^{\gamma} \mathrm{e}^{\gamma} \mathrm{e}^{\gamma} \mathrm{e}^{\gamma} \mathrm{e}^{\gamma} \ldots
$$

where a photon $(\gamma)$ is the gyrolink and the electron is the gyromodule. This model thus boldly contrasts with the current notion that electrons reside as either unpaired or paired entities. The mobilization of the hydrogen electron (primary electrogyre) or any other sole electron to and from other electronexuses (secondary electrogyre) is modeled thusly:

$$
\mathrm{e}^{2 \gamma}+\oplus \leftrightarrows \mathrm{e}^{3 \gamma} \leftrightarrows\left[\mathrm{e}^{\gamma}\right]+2 \oplus
$$

Compressing the gyrequation, I have

$$
\mathrm{e}^{2 \gamma}+\infty \leftrightarrows\left[\mathrm{e}^{\gamma}\right]+2 \oplus
$$

and balancing gives the new gyrequation,

$$
\mathrm{e}^{2 \gamma} \leftrightarrows\left[\mathrm{e}^{\gamma}\right]+\infty
$$


This $1^{\circ} / 2^{\circ}$ schema illustrates the singularity $\left({ }^{\oplus}\right)$ as metabolizing the electronexus. The further IEM is from the singularity, the lesser the attractorepulsive effect on it. In other words, the more distal IEM has a reduced electronexus cycle rate and forms longer, more stable electronexuses. This gyromodular organization thus explains the origin and emergence of atomic orbitals of all chemical elements and why there exist a greater number of electrons $(2,8,18,32 \ldots)$ in outer orbitals, or "shells [77]." The singularity, modeled dextrally to homeostatically balance the left-handed electrogyre, is predicted to induce electronexuses to form right-handed helices $\left(\mathrm{G}_{\mathrm{XII}}\right)$ called electrohelices. Because an electrohelix in one atom exerts attractorepulsive effects (the electrohelix is a gyrating system) on free electrons and electrohelices in other atoms, this gyrosystem explains the emergence of inorganic chemicals that are necessary for the origin and evolution of life [78,79].

Fermi Gas and Liquid States. When visible energy is extracted from or unavailable for the electrogyre, the particles stop cycling between gyrosystates - this models a Fermi gas [80,81]. The Fermi liquid state, presently modeled mathematically (for instance, [82]) is modeled here as a three-dimensional crosslinked network of gyrobasal electrohelices-constantly undergoing metabolism, thus explaining fluctuating quantum "stripes" $[83,84]$ - that are predicted to form a matrix, an electromatrix. Fermi gases and liquids bear the signature of the electrogyre in their dynamic vortices [85-89].

Electromagnetism. Electromagnetism is a fundamental force of nature [90]. The primary electrogyre affords a new view of how this force emerges in the universe: repulsion of photons from and by the photonic singularity onto the electron depicts electricity,

$$
\mathrm{e}^{2 \gamma}+\infty \rightarrow \mathrm{e}^{3 \gamma}
$$

whereas photonic attraction into the singularity depicts magnetism,

$$
\mathrm{e}^{3 \gamma} \rightarrow \mathrm{e}^{2 \gamma}+\infty
$$

Thus, electro-magnetism can be rewritten as gyral repulsion-attraction [91]. Since the attractorepulsive force of any one electrogyre adapts to another via its singularity, the electrogyre models how changes to a magnetic field generate an electric field and vice versa. In response to incoming visible energy, the photonic singularity expands within a spatiotemporally restricted electrogyre. At a critical threshold, this expansion causes electrogyre collapse, accompanied by a quantized photon emission that thermodynamically flows and fractalizes through proximal electrogyres. Planetarily, this models a lightning strike and pre-lightning emissions [92-94]. Furthermore, given that the electrogyre expands omnidirectionally from within to without, it manifests as a high-energy, unstable electromagnetic sphere (electrosphere) that filled with light; this explains the enigmatic nature of ball lightning [95,96].

Quantum Gravity. The electrogyre unifies QM and GR. QG is modeled as the attractive force of the photonic singularity (a spacetime vortex itself $\left(\mathrm{G}_{\mathrm{I}}\right)$ ) on the electron particle. The enigmatic wave-particle complementarity [97] of the electron is clarified because a gyrating particle oscillating between two states, creating the waveform $\left(\mathrm{G}_{\mathrm{II}}, \mathrm{G}_{\mathrm{V}}\right)$. Moreover, the electrogyre (Figure $2 \mathrm{a}$ ) shows how an experimentalist can examine quantal (particle) properties or the gyre (wave) properties, but not both at the same time. Given that the electron oscillates near the speed of light, the gyrostates of one electrocycle are difficult to detect but are predicted by theory. Electron observation requires photons. 
Examination induces visible energy exchange in the gyradaptor and, as long as photonic input is maintained, the particle arrests its gyratory motion (collapses its wavefunction) in the gyrapical state; this explains the measurement problem $[98,99]$. The Heisenbergian uncertainty of knowing two distinct particle characteristics simultaneously (e.g., position and trajectory [100]) is explained by the generic gyromodel itself (Figure 1b; 2.4.2.) and by the innate adaptation (electrogyre learning is electrognosis; electrogyre memory is electromnemesis) of the gyrosystem in response to interrogation. Given that the electrogyre is a theoretical solution to QG, it affords a radical perspective on the core elements of reality and on the primal role of gravity in the evolution of life [101,102].

Planetary core. The current idea for how planets originate in the cosmos, in medias res, is through the cooling of an interstellar gas cloud followed by the gravitational accretion of particles into larger and larger aggregates. As the story goes, the gravitational sink of aggregates leads to accelerative accretion and the emergence of protoplanets [103]. High-pressure and temperature experiments, seismology, and fluid dynamics modeling have led scientists to infer properties of Earth's core [104] and to make statements about the origin of Earth in particular [105]. However, perhaps apocryphally, Einstein considered geomagnetism one of the most important unsolved problems of physics, implicitly calling the accepted model into question.

Using the gyromodel, the inner core of a planet is modeled as a macroelectrogyre, such that, for example,

$$
\left(\mathrm{e}^{3 \gamma} \leftrightarrows \mathrm{e}^{2 \gamma}+\infty\right)_{\mathrm{n}} \text { and }\left(\mathrm{e}^{3 \gamma} \leftrightarrows\left[\mathrm{e}^{\gamma}\right]+2 \infty\right)_{\mathrm{n}}
$$

where (1) models a macrophoton, and $\mathrm{n}$ is an inordinately large number of components in the gyrosystem. This gyrequation reveals that the planetary core (macroelectron) emerges from a macrophoton in a fashion similar to leptonic emergence from a photon. As the macroelectrogyre has the vectorial, spinning, flowing form, it is compatible with the geophysical evidence regarding precession [106] — where a planet rotates on its own axis just like a spinning gyroscope [107] — and the geomagnetic field [108], which is modeled cosmically as it is atomically (Figure 4a). Further, because the excited state of the macroelectrogyre is shared by all the majorgyres, the secondary electrogyre-derived macroelectronexus emerges within and models the inner core of a planet. Being an adaptive supermassive helix, the macroelectronexus is consistent with the notion of a geodynamo in Earth's core [109] yet diverges from the current idea that the core is liquid iron [110]. Finally, the gyrobase of the tertiary electrogyre (Figure 2a (iii)) models a planetary core, [e], that loses its macrophotonic support. The thermodynamic switch between the two most extreme majorgyrostates $\left(\mathrm{e}^{3 \gamma}\right.$ and e) parsimoniously models geomagnetic reversal, a periodic geophysical event that has not been observed and thus has engendered much speculation [111-113].

Planetary orbit. As the macroelectrogyre (planet) spins on its own axis as a consequence of the thermodynamic flow from its internal macrophoton, it rotates in an observable higher-order gyre (ohiogyre) around a central, capacious, more exergic macrophoton (star) from which it emerged. The ohiogyre provides a unique perspective on how energy emitted by a star influences the evolution of a planet: macrophoton expulsion (solar wind [114-116], coronal mass ejections [117,118]) repels the macroelectrogyre into a high energy state known in celestial mechanics as perihelion. The macroelectrogyre adapts by mobilizing, metabolizing, storing, and changing the energy within itself and expanding. Being a dissipative system, the macroelectrogyre also disperses some of the energy as 
heat into space, thereby falling to a relativistically lower energy state known as aphelion. Thus, as modeled by the ohiogyre, quantized macrophoton influx induces macroelectrogyre oscillation between excited and ground states, explaining both the periodicity of planetary orbit and why a planet does not gravitationally collapse into a star. Finally, as with atomic orbitals, in planetary orbits, the attractorepulsive effects diminish the further away from the macrophoton singularity. The macroelectrogyre predicts that increased size and slower orbit of distal planets relative to proximal ones (as in the Solar System) corresponds to the composition, length, and stability of macroelectronexuses.

Antimatter. An outstanding question in physics is why there is so little antimatter in the physical universe $[119,120]$. Microcosmically, the tertiary electrogyre (Figure 2a (iii)) shows the electron cycling out the thermodynamic support of the triphoton. Given synchiral organization of the tertiary majorgyre gyrobase $\left(\mathrm{G}_{\mathrm{VII}}, \mathrm{G}_{\mathrm{XII}-2}\right)$, the electron destabilizes and ultimately collapses due to the synchiral sub 2 gyre (not shown) in lieu of the antichiral subgyre, modeling the positron. The extreme creatodestructive swing of the electrogyre thus provides an explanation for the fleeting presence, or absence, of antimatter in the universe.

From Visible Matter to Water. The electrogyre models, explains, positions, and predicts fundamental physical phenomena and provides a framework for the origin and evolution of the Solar System in the Milky Way Galaxy [121]. Although I intentionally focused on data acquired from experiments and observations of the Solar System, the electrogyre is flexible enough to be tested against evidence regarding any star and planetary system in the universe [122]. Compressing the primary, secondary, and tertiary electrogyres reveals how leptons and photons interconvert:

$$
\mathrm{e}^{2 \gamma} \leftrightarrows\left[\mathrm{e}^{\gamma}\right]+\infty \leftrightarrows[\mathrm{e}]+2 \oplus
$$

Importantly, this empirically established and symbolically represented relationship precisely conforms to the first law of thermodynamics, known as the physical law of the conservation of energy [123].

The universe has been expanding since its origin and universal expansion is currently accelerating [124]. I explain this expansion (i.e., cosmological inflation [125]) as the omnidirectional repulsive force of the photon on the electrogyre and, consequentially, as the repulsive force of the electrogyre on all of the supervenient forms of matter and information in the evolving universe. (I qualify my explanation by noting that this framework does not incorporate or explain dark energy, dark matter, and sub-atomic particles [126-128].) This theory predicts that, during expansion of the universe, the electrogyre - an ordered mélange of elements and inorganic chemicals - achieved a thermodynamically unstable state of high energetic potential, whereupon, the electrogyre collapsed. This disruption and release of kinetic energy, in light of continued expansion, predicates a significant, far-reaching change in universal evolution: the emergence of water.

\subsection{Water}

The emergence of novel IEM forms is a grand evolutionary and philosophical problem $[129,130]$. Theoretically solving this problem should, in principle, be possible, since evolutionary events are constrained by natural laws, physical forces, and chemical elements that lead up to them. Yet biological macroevolution is thought to work quantally, "explosively [131]." At this juncture, I require 
an answer to the following question: What fundamental feature of life evolves following and from visible, inorganic matter?

As water is the single largest component (70-90\%) of the living cell, the fittest answer is water [132]. Known as "the universal solvent," water is one of the simplest chemical molecules, consisting of oxygen $(\mathrm{O})$ and hydrogen $(\mathrm{H})$. In spite of its chemical simplicity, its complexity is legendary: a brutally honest, erstwhile Nature editor opined, "no one understands water [133]." In this subsection, I squarely face the emergence of water with a reified systems model called the oxygyre. The oxygyre not only models the origin and nature of water in the universe and in the living cell but also fits data related to celestial oxides, oceans, and moons.

I make two crucial points prior to proceeding. First, in modeling the chemistry and thermodynamics of water, the reader must view the hydrogen atom from the standpoint of its sole electron (denoted here as the quantized particle (e); Figure 2a (iv)) rather than its proton (D). Second, given theoretical expansiveness and particle-quantum relations $\left(\mathrm{G}_{\mathrm{III}}\right)$, e has alternate applications beyond the hydrogen electron; these are discussed later in this subsection.

Origin of water. There have been many clues and ideas regarding the origin of water on Earth and in the universe [134-136], but no solution. Modeling the origin of water here requires a brief reminder of electrogyre characteristics (3.1). As the singularities of the primary and secondary electrogyre are uniphoton (\$) and diphoton (2\$), respectively (Figures 2a (i) and (ii)), the singularity of the secondary electrogyre exerts a greater attractive force on the triquantal excited state electron $\left(\mathrm{e}^{3 \gamma}\right)$ than does that of the primary electrogyre (recall that majorgyres share the triquantal state). Thus, the electrophilicity of the oxygen atom, like all other electron sinks, is modeled by the relativistically higher magnetism of the secondary electrogyre. Moreover, as gyromodeled, the relative proximity of an electron or electronexus to the singularity affects its metabolic rate.

The gyromodel dictates that the oxyon (the quantum/particle form of the oxygyre, Figure $2 b(i v)$ ) particle spins levorally yet gyrates dextrally to offset electrogyre chirality $\left(\mathrm{G}_{\mathrm{VI}}, \mathrm{G}_{\mathrm{VII}}\right)$ - thereby diminishing the rate of gyrostatic oscillations and homeostatically balancing the gyrosystem and the universe. In other words, the chemical molecule known as water emerges quantally from within the pool of elements during the aforementioned electrogyre collapse. Gyromodelling water would thereby be axiomatically compatible: a focagyre (oxygyre) is dependent upon a subgyre (electrogyre) and is an emergent IEM form ( $\left.\mathrm{G}_{\mathrm{VIII}}, \mathrm{G}_{\mathrm{IX}}\right)$.

Phase transitions. Where chemical molecules are concerned, there are three main phases, or distinct states of matter, which have essentially uniform physical properties throughout: solid, gas, and liquid. The current approach for describing phase transitions - thermodynamic transformation from one state of matter to another - is mathematical [137]. Despite much progress, the math is incapable of explaining why there are three main phases and precisely how they interconvert. Here, I fit evidence about phase transitions of water to the majorgyre frame.

Microcosmically, the primary oxygyre (Figure $2 \mathrm{~b}(i)$ ) models the molecular oscillation between $\mathrm{H}_{3} \mathrm{O}\left(\mathrm{O}^{3 \mathrm{e}}\right.$; gyrapex $)$ and $\mathrm{H}_{2} \mathrm{O}\left(\mathrm{O}^{2 \mathrm{e}}\right.$; gyrobase $)$. Unfortunately, confusing matters slightly, this is written in chemical notation as

$$
\mathrm{H}_{3} \mathrm{O}^{+}+\mathrm{e}^{-} \leftrightarrows \mathrm{H}_{2} \mathrm{O}+\mathrm{H}
$$


because the trielectron oxygen $\left(\mathrm{H}_{3} \mathrm{O}\right)$ is a challenge to detect due to its instability $\left(\mathrm{H}_{3} \mathrm{O}^{+}+\mathrm{e}^{-}\right)$. This gyrosystem parsimoniously depicts sublimation (transformation from solid to gas) as attraction by the singularity, pulling the electron off the oxygen atom and reconstructing the hydrogen atom (e); gyradaptor):

$$
\mathrm{O}^{3 \mathrm{e}} \rightarrow \mathrm{O}^{2 \mathrm{e}}+\odot
$$

Deposition (transformation from gas to solid) is modeled as repulsion of the electron onto oxygen:

$$
\mathrm{O}^{2 \mathrm{e}}+\mathrm{e} \rightarrow \mathrm{O}^{3 \mathrm{e}}
$$

Thus, $\mathrm{O}^{3 \mathrm{e}}$ represents ice and $\mathrm{O}^{2 \mathrm{e}}$ is water vapor. The primary oxygyre models these phase changes in living systems [138] and, as it is a general theory, on a macrocosmic scale in glaciers, hail, sleet, snow, clouds, and fog in both early evolution of planets and present-day atmospheres [139,140], i.e.:

$$
\left(\mathrm{O}^{3 \mathrm{e}} \leftrightarrows \mathrm{O}^{2 \mathrm{e}}+(\mathrm{e})_{\mathrm{n}}\right.
$$

Understanding the other phase and phase transitions of water requires fitting data onto the secondary majorgyre. The gyrobase of the secondary oxygyre (Figure $2 b(i i)$ ) models an oxy-electron monomer ( $\left[\mathrm{O}^{\mathrm{e}}\right]$ fits $\mathrm{OH}$, or hydroxyl ion), dimer (fits $\mathrm{H}_{2} \mathrm{O}_{2}$, hydrogen peroxide), and any length of polymer, an oxynexus, shown as:

$$
\left[\mathrm{O}^{\mathrm{e}}\right]=\mathrm{O}^{\mathrm{e}}, \mathrm{O}^{\mathrm{e}} \mathrm{O}^{\mathrm{e}}, \mathrm{O}^{\mathrm{e}} \mathrm{O}^{\mathrm{e}} \mathrm{O}^{\mathrm{e}} \mathrm{O}^{\mathrm{e}} \mathrm{O}^{\mathrm{e}} \mathrm{O}^{\mathrm{e}} \ldots
$$

As the gyromodel stipulates, oxynexuses are continually being catabolized (created) and anabolized (destroyed) by the electron singularity. Given $G_{\text {XII }}$, the oxynexus is predicted to assemble into a left-handed oxyhelix. What do oxynexuses and oxyhelices model? These structures represent how the liquid phase of water is organized [141,142]. Large-scale ordering of these oxynexuses are matrices, called oxymatrices; on a planetary scale, a macroxymatrix is the theoretical term for an ocean. Given the theoretical finding that macroxymatrices are a direct evolutionary and thermodynamic consequence of the macrophoton (Sun) and macroelectron (Earth), this helps unravel the faint young sun paradox $[143,144]$. The secondary oxygyre models melting (solid to liquid) as gyradaptive attraction by the dielectron:

$$
\mathrm{O}^{3 \mathrm{e}} \rightarrow\left[\mathrm{O}^{\mathrm{e}}\right]+2 \odot
$$

Freezing (liquid to solid), in contrast, is gyradaptive repulsion by the dielectron:

$$
\left[\mathrm{O}^{\mathrm{e}}\right]+2 \odot \rightarrow \mathrm{O}^{3 \mathrm{e}}
$$

Combining the primary and secondary oxygyre in a gyrequation gives

$$
\mathrm{O}^{2 \mathrm{e}}+\bigodot \leftrightarrows \mathrm{O}^{3 \mathrm{e}} \leftrightarrows\left[\mathrm{O}^{\mathrm{e}}\right]+2 \bigodot
$$

thus tidily modeling the interconnectivity of the three phases of water in the biogeochemical water cycle,

$$
\mathrm{H}_{2} \mathrm{O}+\mathrm{H} \leftrightarrows \mathrm{H}_{3} \mathrm{O} \leftrightarrows \mathrm{OH}+\mathrm{H}_{2}
$$

termed here a macroxygyre: solid $\left(\mathrm{O}^{3 \mathrm{e}}\right)$, gas $\left(\mathrm{O}^{2 \mathrm{e}}\right)$, and liquid $\left(\left[\mathrm{O}^{\mathrm{e}}\right]\right)$. One full cycle of any oxygyre is called an oxycycle. Therefore, this model explains the biogeochemistry of water ([145], and see below) and is a heuristic for framing phase transitions for any other forms of visible matter. Please note the 
modeling of hydrogen gas (where 2e $=\mathrm{H}_{2}$ ), an important molecule in chemosynthesis and planetary organization and formation [146-149]. Compressing the gyrequation, I have

$$
\mathrm{O}^{2 \mathrm{e}}+\odot \leftrightarrows\left[\mathrm{O}^{\mathrm{e}}\right]+2 \odot
$$

and balancing reveals

$$
\mathrm{O}^{2 \mathrm{e}} \leftrightarrows\left[\mathrm{O}^{\mathrm{e}}\right]+\odot
$$

providing a theoretical schema for the gas-liquid phase transition. I use this specific transition to illustrate the electron-as-singularity concept (Figure 3b). Rounding out the phase transitions of water, condensation (gas to liquid) is modeled as electron attraction from the oxygen electronexus $\left(\mathrm{O}^{2 \mathrm{e}}\right)$ into the singularity (e), forming liquid water $\left(\left[\mathrm{O}^{\mathrm{e}}\right]\right)$ :

$$
\mathrm{O}^{2 \mathrm{e}} \rightarrow\left[\mathrm{O}^{\mathrm{e}}\right]+\odot
$$

Vaporization (liquid to gas) is modeled as particle repulsion from the singularity to the oxygen electronexus, orbiting the atomic nucleus:

$$
\left[\mathrm{O}^{\mathrm{e}}\right]+\odot \rightarrow \mathrm{O}^{2 \mathrm{e}}
$$

Reiterating, as conjunctively modeled by the oxygyre, attractorepulsive electron flow through the $\mathrm{O}$ orbitals is liquid (fluid water); electron capture by $\mathrm{O}$ is solid (ice); electron eviction by $\mathrm{O}$ is gas (water vapor).

Nature of water. I now discuss a few of water's enigmatic characteristics in light of the model. First, as the oxygyre expands and contracts omnidirectionally in response to an influx of visible energy within the electron singularity (Figure $2 \mathrm{~b}(i v)$ ), the oxygyre concomitantly fills out the hyperbolic geometry of a sphere (oxysphere). The binary attractorepulsive effects-photon-electron, electron-oxyon, and photon-oxyon - explain not only the uniform but adaptive spherical shape of a water droplet but also the general surface tension of water [150,151]. Second, because a gyrating oxyon particle assembles into higher-order quantized structures that also gyrate and oscillate $\left(\mathrm{G}_{\mathrm{II}}\right.$, $\mathrm{G}_{\text {III-1 }}$, and $\mathrm{G}_{\mathrm{XII}}$ ), this provides a theoretical basis for the tempo and mode of the water oscillatory waveform [152,153]. Third, the controversial if scientifically heretical concept that water has memory [154,155] is supported by theory, as the oxygyre undergoes oxygnosis and oxymnemesis as part of its adaptational cycle (2.4.4.). Fourth, Matrioshkagyre relationships of the photon, electron, and the oxygyre demonstrate how visible energy is stored within water and explain the high heat capacity of water - an alternative view than that given by mathematical models [156]. Fifth, the hemispherically antichiral oceanic gyres in the Atlantic and Pacific oceans [157,158] are modeled as a consequence of the attractorepulsive forces exerted by the vectorial macroelectronexus at the core of the Earth on the macroxymatrix. Sixth, the vortical structure seen throughout the oceanic and atmospheric world (e.g., hurricanes, maelstroms, tornados) is modeled by the oxygyre and is even detectable in nano-ice (Figure 4b; [159]). 
Figure 3. Understanding singularities. (a-d, f-i) Each singularity (gyre center) is represented as follows: (i) Gyrosystem; (ii) Matrioshkagyre; (iii) bidirectional, linear reaction or process; (iv) gyrequation. (a) Primary $\left(1^{\circ}\right)$ electrogyre (b) Alternoxygyre (c) Primary $\left(1^{\circ}\right)$ carbogyre (d) Alternaphosphogyre; $\mathrm{n}=$ any positive integer; $\mathrm{P} \sim \mathrm{P}$ is pyrophosphate (e) Matrioshkagyre of the presented electro-, oxy-, carbo-, and phosphogyres (f) Secondary $\left(2^{\circ}\right)$ ribogyre $\left(\right.$ g) Tertiary $\left(3^{\circ}\right)$ aminogyre. Translation apparatus is the same as aa-3RNA (h) Alternagenogyre (i) Hapcellulogyre. Here, $1 \mathrm{~N}$ and $2 \mathrm{~N}$ represent chromosome content (j) en face Matrioshkagyre of the presented ribo-, amino-, geno-, and cellulogyres. Note how the Matrioshkagyre form reveals the nested thermodynamics and accurately positions one physical, chemical, biochemical, or biological process related to another. Acronyms, symbols, and models are defined in Tables 1 and 2, Figures 1 and 2.

$\mathbf{a}(i)$

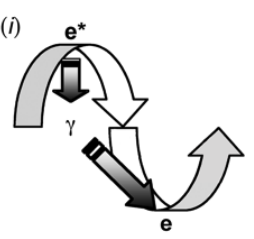

(ii)

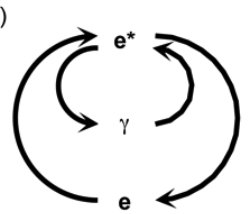

(iii)

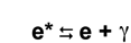

(iv)

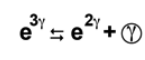

(iii)

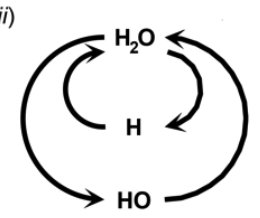

(ii)
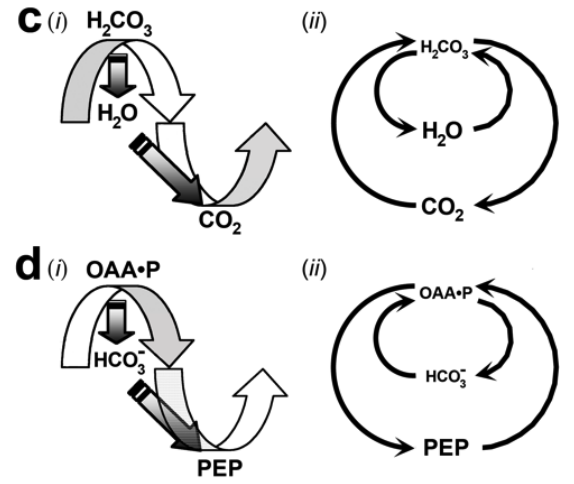

(ii)

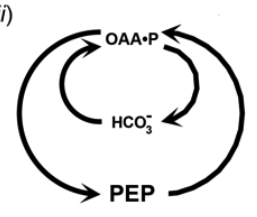

(iv)

(iii)

(iv)

(iii)

(iv)

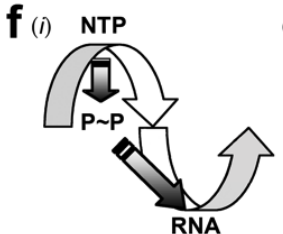

(ii)

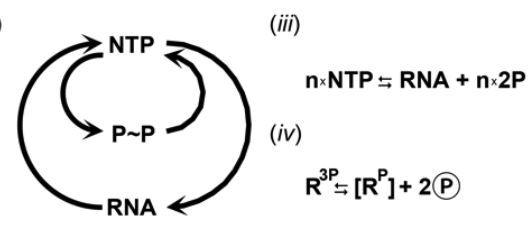

$\mathrm{H}_{2} \mathrm{O} \leftrightarrows \mathrm{OH}^{-}+\mathrm{H}^{+}$

$\mathrm{O}^{2 \mathrm{e}}=\left[0^{\mathrm{e}}\right]+(\mathrm{e}$

$\mathrm{H}_{2} \mathrm{CO}_{3} \leftrightharpoons \mathrm{CO}_{2}+\mathrm{H}_{2} \mathrm{O}$

$c^{30} \leftrightarrows c^{20}+(0)$

$\mathrm{C}_{4} \mathrm{H}_{4} \mathrm{O}_{5}+\mathrm{HPO}_{4}^{2^{-}} \leftrightarrows$

$\mathrm{C}_{3} \mathrm{H}_{5} \mathrm{O}_{6} \mathrm{P}+\mathrm{HCO}_{3}^{-}$

$P^{4 C} \leftrightarrows P^{3 C}+(C)$

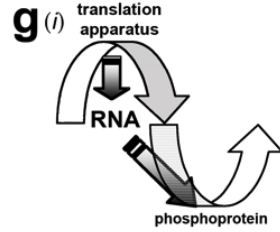

$\mathbf{h}_{(i) \mathrm{n}}$
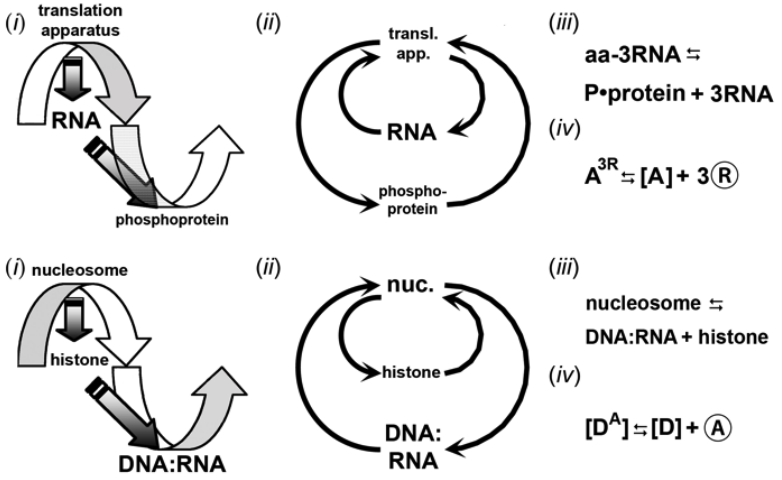

I (i) $\quad 2 \mathrm{~N} \quad$ (ii) (iii)

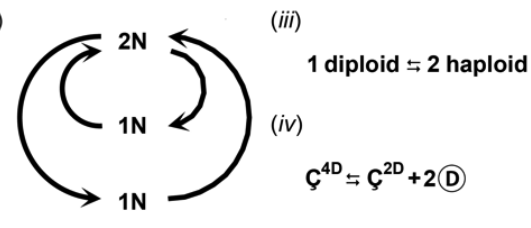

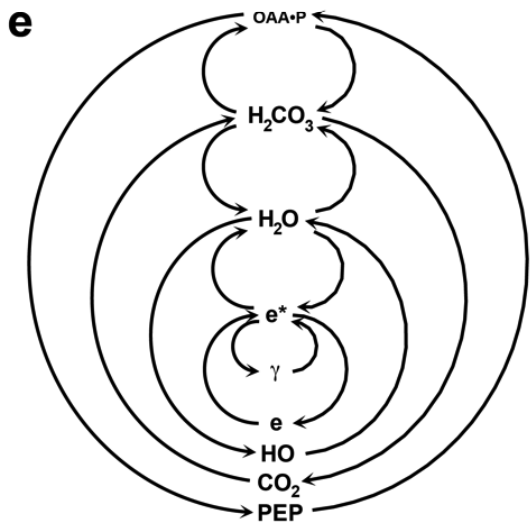

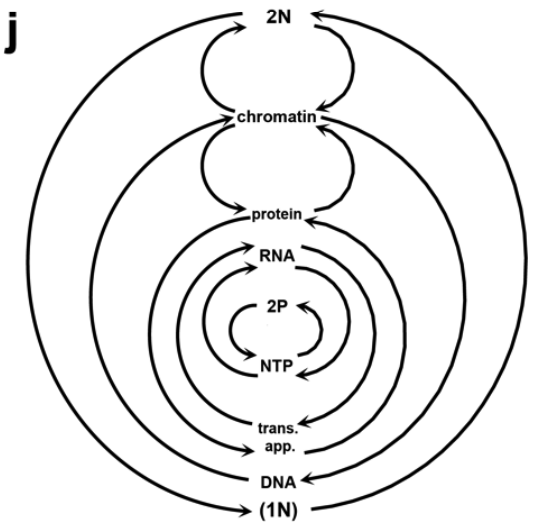

Oxygen species. The origin of oxygen in early Earth's atmosphere is thought to be a solved problem: cyanobacteria emerged in an anoxic environment to produce molecular oxygen $\left(\mathrm{O}_{2}\right)$ as a byproduct of photosynthesis $[160,161]$. However, since there is no way of performing experiments on 
early Earth, this idea cannot be directly validated or refuted. Departing from this provisional solution, I applied the oxygyre to the problem. The tertiary oxygyre (Figure $2 \mathrm{~b}$ (iii)) depicts trielectron cycling from, to, and through the singularity, revealing how the oxygen species-elemental oxygen and reactive oxygen radical, $\mathrm{O}$ or $[\mathrm{O}]_{1}$, molecular oxygen, $\mathrm{OO}$ or $[\mathrm{O}]_{2}$, and ozone, $\mathrm{OOO}$ or $[\mathrm{O}]_{3}$ - originate and oscillate to and from the solid phase of water $\left(\mathrm{O}^{3 \mathrm{e}}\right)$ :

$$
\mathrm{O}^{3 \mathrm{e}} \leftrightarrows[\mathrm{O}]+3 \odot
$$

Note that, the photon is the gyrolink between the oxygen gyromodules $\left(\mathrm{G}_{\mathrm{XI}}\right)$, thus providing insight into how the oxygen-dense atmosphere adapts to the Sun's efflux [162]. Moreover, the tertiary oxygyre dictates an early emergence of oxygen species on Earth and their necessity to the evolutionary origin of life $[163,164]$. Modeling the movement of water vapor through living systems and the atmosphere in the oxygen cycle (another macroxygyre) requires combining the primary and tertiary oxygyres,

$$
\mathrm{O}^{2 \mathrm{e}}+\odot \leftrightarrows \mathrm{O}^{3 \mathrm{e}} \leftrightarrows[\mathrm{O}]+3 \odot
$$

which compresses and balances to

$$
\mathrm{O}^{2 \mathrm{e}} \leftrightarrows[\mathrm{O}]+2 \mathrm{e}
$$

uncovering the $1 \% 3^{\circ}$ alternagyre. Modeling of the relationship of liquid water and oxygen species is modeled by compressing secondary and tertiary oxygyres:

$$
\left[\mathrm{O}^{\mathrm{e}}\right]+2 \odot \leftrightarrows \mathrm{O}^{3 \mathrm{e}} \leftrightarrows[\mathrm{O}]+3 \odot
$$

which reduces and balances to

$$
\left[\mathrm{O}^{\mathrm{e}}\right] \leftrightarrows[\mathrm{O}]+\text { ( ) }
$$

revealing the $2^{\circ} / 3^{\circ}$ alternagyre. Finally, given that the tertiary oxygyre oscillates between extreme gyrostates $\left(\mathrm{O}^{3 \mathrm{e}}\right.$ and $\left.[\mathrm{O}]\right)$ over a geological time scale, this explains not only why and how Earth experienced several intervals of intense glaciation $\left(\mathrm{O}^{3 \mathrm{e}}\right)$ known as "snowball Earth $[165,166]$ " but also the appearance of free oxygen $\left([\mathrm{O}]_{2}\right)$ in Earth's atmosphere, like the "great oxidation event [167]."

Oxide Geochemistry and Geophysics. The necessity of modern geochemical cycles to bio-organismal existence on Earth conceals a vital thermodynamic connection between planetary and cellular evolution [168]. Still, it is inappropriate to harbor any assumptions related to such connections but allow theory to inform them. I reported in 3.1 that the macroelectron, the planetary inner core, harbors within itself the potential for all chemical elements and inorganics. I now frame a second major leap in planetary evolution: the formation of the outer core, mantle, and crust.

The Earth's mantle and crust are highly enriched in oxides, all of which are accounted for by the primary and secondary oxygyre. For example, in the primary oxygyre, $\mathrm{O}^{2 \mathrm{e}}$ - where e here represents the secondary electrogyre and hence any chemical elements $\left(\mathrm{G}_{\mathrm{III}}\right)$ - models the low abundance compounds $\mathrm{Na}_{2} \mathrm{O}$ and $\mathrm{K}_{2} \mathrm{O}$ (each ion represents $1 \mathrm{e}$ and thus two make the compound); it also models $\mathrm{CaO}, \mathrm{FeO}$, and $\mathrm{NaO}$ (each ion represents $2 \mathrm{e}$ ), which are $\sim 3,7.5$ and $48 \%$ of the Earth's mantle and $\sim 6$, 7 , and $5 \%$ of the crust, respectively [169]. The secondary oxygyre also models the major makeup of Earth's outer core [170], mantle, and crust. As the outer core has been proposed to be liquid [171], a macroxygyre models its thermodynamically fluid and dynamic character. $\left[\mathrm{O}^{\mathrm{e}}\right]_{2}$ models $\mathrm{SiO}_{2}$, this being $\sim 46 \%$ of the mantle and $\sim 61 \%$ of the crust and $\left[\mathrm{O}^{\mathrm{e}}\right]_{3}$ accounts for $\mathrm{Al}_{2} \mathrm{O}_{3} \sim 4 \%$ of the mantle and 
$\sim 16 \%$ of the crust [169]. Supporting a more broad application of the oxygyre to understanding crustal organization and dynamics, oxygen is $\sim 50 \%$ of crustal mass and the crust itself is $>99 \%$ oxides [172].

Geophysical theories have been quite successful, but anomalies and inexplicable phenomena have hinted at their limitations [173]. I submit that there are several noteworthy geophysical features that can be deduced from the nesting of the macrophoton within the macroelectron within the macroxyon. First, in response to photon influx from solar emissions, the macrophoton swells within the macroelectron. As photons step-decelerate to leptons (3.1), the macroelectron, in turn, organizes, stores, and emits IEM from within to without. Macroelectron expansion elicits macroxyogyre expansion to accommodate the IEM influx. This theoretical scheme prescribes that the Earth - and, as predicted by theory, all planets and planetary bodies - formed by expansion as opposed to accretion [174]. This prescription conforms with the expanding Earth concept [175] and thus addresses numerous problematical issues in the fields of volcanism [176,177], landmass formation [178-180], continental drift [181], and seismology [182,183]. On the most latter point, I elaborate on how the Matrioshkaquantal structure of the Earth relates to seismic activity. During expansion, the macrophoton singularity (within the macroelectron core) reaches a local thermodynamically unstable state, detected as pre-earthquake signals [184], whereupon it re-equilibrates. A quantal emission of energy ripples outward, moving as focused solitons [185] through the macroelectron and macroxyon layers, i.e., spherical shells. Following passage through these gyrosystems on macrocosmic and microcosmic scales (all of the unique chemical elements and molecules throughout the inner and outer core, mantle, and crust), the quantum of energy arrives at a spacetime point of criticality. The unpredictability, fractalization, and rippling solitonic flow of gyrosystems resolves the enigmatic characteristics of earthquakes [186,187]. Here stands a formal theoretical relationship between solar emissions and seismic activity, confirming a long-standing idea [188].

Lunar Formation. The favored hypothesis for the formation of Earth's Moon is from planetesimal impact on a proto-Earth proceeded by matter ejection, accretion, and gravitational capture [189,190]. However, the question of lunar origin has not been settled since there are competing, albeit antiquated hypotheses [191,192]. I also discovered the stunning admission that, “...shamefacedly, [astronomers] have little idea as to where [the Moon] came from. This is particularly embarrassing... [193].” The oxygyre models the Moon as a macroxyon that has a macroelectron within itself; this simple gyrosystem accounts for the known chemical composition of the Moon surface, oxides [194]. Regarding lunar origin, the macroxyon that is the Moon emerges from the macroelectron that is the Earth, concomitant with the emergence of Earth's macroxyon $[195,196]$.

Several additional points can be derived from this gyrosystem. First, the oxygyre explains water on and in the Moon [197-199]. Second, the gyrating effects of the macroxygyre model the rotation of the Moon on its axis. Third, the path of a less exergic macroxyon (Moon) around more exergic one (Earth) follows an ohiogyre path, or lunar orbit. Fourth, this oxygyre provides insight into how tidal cycling is linked to lunar orbit and axial rotation [200] since the Earth's oceans (macroxymatrix) and Moon itself (a macroxyon) exert complementary attractorepulsive forces. Fifth, this theoretical union also helps clarify short-term chronobiological ([201]; see 3.8) and long-term geophysical [202] relationships. Sixth, the craters that cover planetary, lunar, and satellite surfaces [203-205] — most if not all of which are near-perfect circles-bear the signature of the macroelectron singularity and its strong thermodynamic force on the oxygyre [206]. 
From Phased Matter to Organic Matter. In this subsection, I restricted my attention to Earth for a very important reason: life as I know it evolved on Earth and thus Earthly life is what I model. This rationale guides fact fitting in subsequent subsections. Still, the oxygyre is a cohesive and consistent model for the emergence and cycling of oxygen species in the early evolution of the Earth [207,208], different from any computer model [209]. The oxygyre is predicted to be fruitful vis-à-vis modeling planets and moons of the Solar System and Earth-like planets and star systems throughout the cosmos [210,211].

Figure 4. Gyrosystem Forms. (a) Electrogyre. Atomic chirality pictorially represented (oblique view) as electron probability current density for a hydrogenic $2 p_{1 / 2}$ stationary state Reprinted and minimally adapted with permission from [212]. (C) 1998 American Association of Physics Teachers. (b) Oxygyre. A snapshot of quenched molecular coordinates of nano-ice. Reprinted from [213]. (C) 2006 by The National Academy of Sciences of the USA. (c) Carbogyre. Amylopectin, or glucose polymers with $\alpha(1 \rightarrow 4)$ glycosidic bonds. Stick (left) and space-filling (right) models show how glucose polymers assemble into antiparallel helices. Reprinted from [214] with permission from Wiley. (C) 2010 WILEY-VCH Verlag GmbH \& Co. KGaA, Weinheim. (d) Phosphogyre. Crystal structure of $\gamma-\mathrm{Ca}\left(\mathrm{PO}_{3}\right)_{2}$ showing unidirectional helical polyphosphate chains stacked in alternating perpendicular directions; $\mathrm{Ca}$, blue; O, red; $\mathrm{PO}_{4}$ tetrahedra, green. Reprinted from [215] with permission from Wiley. (C) 2005, American Chemical Society. (e) Ribogyre. Composite structure of 16S rRNA compiled by comparing vacant Escherichia coli and tRNA-occupied T. thermophilus ribosomes. Note how the RNA right-handed double helices compactify into a matrix. Reprinted from [216] with permission from Elsevier. (f) Aminogyre. Crystal structure of the RNA exosome complex is a cyclical hexamer of $\alpha$-helix dense RNase PH subunits. Reprinted and minimally adapted from [217] with permission from Elsevier. (g) Genogyre. Nucleosome architecture is a right-handed DNA double helix wrapping in a left-handed manner around a histone octamer. Reprinted by permission from Macmillan Publishers Ltd: Nature [218], (C) 1997. (h) Cellulogyre. Photograph of Cirripathes spiralis, a coral species. Image by N. Hobgood; licensed under the Creative Commons Attribution-Share Alike 3.0 Unported license.

$\mathbf{a}$

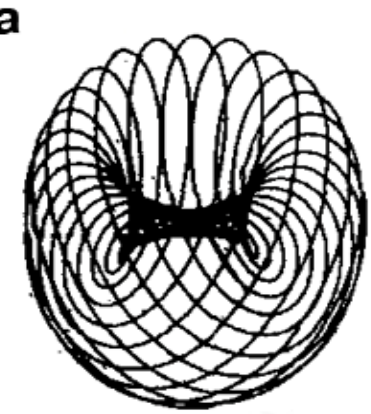

e

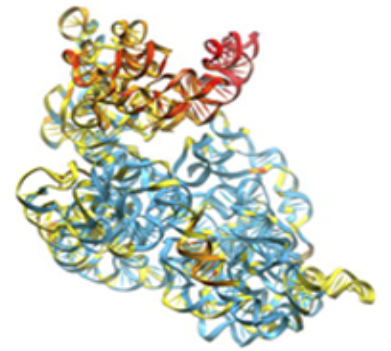

b

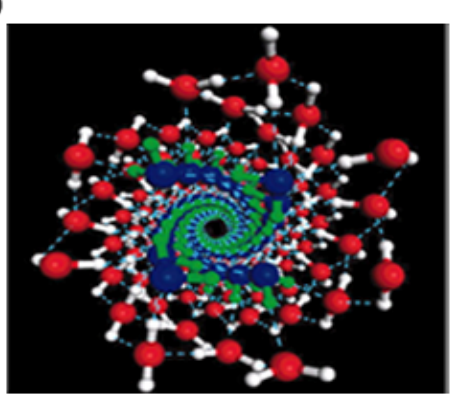

$\mathbf{f}$

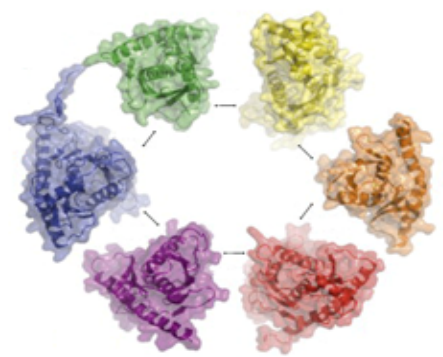

C

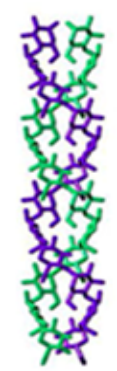

g
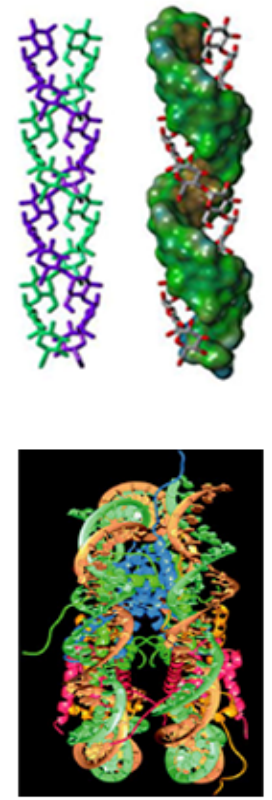

d

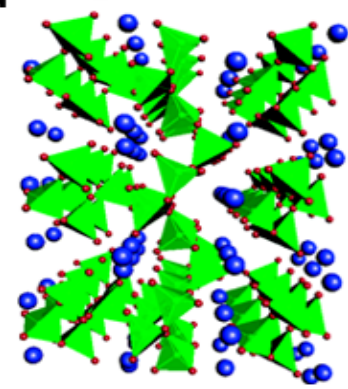

h

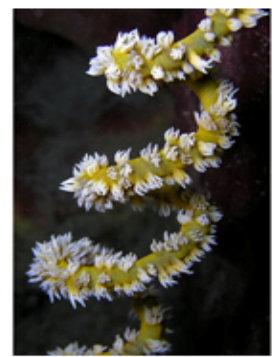


The positioning of information, energy, and matter in the oxygyre relative to the electrogyre accounts for two sets of phenomena originally thought to be modeled independently: modern (present-day) celestial mechanics, geophysical processes, and cellular thermodynamics of water and remote (evolutionary) planetary growth, lunar emergence, and the origins of water and molecular oxygen that are necessary for life's origin. The next gyrosystem must emerge from within the electrogyre and oxygyre; that is, supragyre emergence and thermodynamic metabolism are dependent upon the oxygyre $\left(\mathrm{G}_{\mathrm{VIII}-1}\right)$. On this axiomatic constraint, I fit water and oxide cycling through organic biochemicals and geochemical systems as the next emergence.

\subsection{Organic Matter}

All life that has been identified by the scientific method is carbon-based. In the absence of a consensus explanation for the necessity of organic matter to life, other hypothetical types of biochemistry not reliant on carbon have been postulated (especially silicon, [219]); and yet, none have been identified. Any theory of life, to be considered meritorious, would be expected to provide an explanation for why life is carbon-based and shed light on whether or not alternative biochemistries are possible [220].

Here, I gyromodel evidence regarding carbon compounds on Earth and in the cosmos and regarding organic biochemical pathways in the extant cell. The carbogyre is the gyrosystem that emerges from within the electrogyre and through the oxygyre. In viewing the carbogyre (Figure 2c), the reader should be aware that only two IEM species are permitted in the symbolic architecture of a gyromodel. That is, the carbogyre displays carbon moieties (C), oxygen moieties (particle, O, and quantum, (O) but does not display electrons (e and (e)) or photons (®) - even though they are present-for gyromodel consistency and clarity.

Emergence and Cycling of $\mathrm{CO}_{2}$. The origin of carbon dioxide $\left(\mathrm{CO}_{2}\right)$ in Earth's atmosphere is a matter of much speculation [221,222]. Understanding its origin is of great scientific import, as plants literally construct themselves from, and maintain themselves with, $\mathrm{CO}_{2}$ [223,224]. The primary carbogyre (Figure 2c $(i)$ ), written in the gyrequation

$$
\mathrm{C}^{3 \mathrm{O}} \leftrightarrows \mathrm{C}^{2 \mathrm{O}}+\mathrm{O}
$$

exquisitely fits the chemical reaction

$$
\mathrm{CH}_{2} \mathrm{O}_{3} \leftrightarrows \mathrm{CO}_{2}+\mathrm{H}_{2} \mathrm{O}
$$

which is interconversion of carbonic acid with carbon dioxide and water vapor-relevant both astrophysically and terrestrially [225]. Here, $\mathrm{C}^{3 \mathrm{O}}$ is the gyrapex for all majorgyre, $\mathrm{C}^{2 \mathrm{O}}$ is the gyrobase, and $($ ) is the gyradaptor. Modeling water as the singularity is presented four different ways in Figure 3c. Like the aforementioned gyrapices (triphoton, trielectron), the trioxygenated carbonic acid is an extremely high energy, thermodynamically unstable compound [226]. The cycling of carbon dioxide in the early and present day Earth atmosphere and biosphere is thus vectorially modeled as the attractive force of the oxyon singularity on the mIEM oxyon particle (dehydration reaction):

$$
\mathrm{C}^{3 \mathrm{O}} \rightarrow \mathrm{C}^{2 \mathrm{O}}+\mathrm{O}
$$


formation of carbonic acid is due to the repulsive force of the oxyon (hydration reaction):

$$
\mathrm{C}^{2 \mathrm{O}}+\mathrm{O} \rightarrow \mathrm{C}^{3 \mathrm{O}}
$$

This schema shows that, even though the electrogyre accounts for the interactions between and among the electrons in all elements, the primary carbogyre is a dedicated gyrosystem for modeling interactions between and among carbon and oxygen atoms/compounds. A clearer picture of how water is the singularity of the primary carbogyre can be found in Figure 3c. An explanation of the origin of $\mathrm{CO}_{2}$ requires the introduction of additional carbogyrosystems and is discussed below.

Atmospheric $\mathrm{CO}_{2}$ Levels. Keeling was the first to precisely measure monthly atmospheric $\mathrm{CO}_{2}$ levels on Earth from the middle of the $20^{\text {th }}$ century onward, leading to production of his eponymous curve [227]. Two features of this curve are noteworthy: $\mathrm{CO}_{2}$ levels are both increasing and oscillating. While the increase in $\mathrm{CO}_{2}$ has been argued to be a hallmark of global climate change from burning fuel [228,229], the oscillating levels are thought to reflect the natural $\mathrm{CO}_{2}$ flux into and out of the oceans and biosphere. The carbogyre explains both phenomena simultaneously: macrocarbogyre expansocontraction driven by macroxyon expansocontraction and attractorepulsion models the increasing (expansion) and oscillating (spacetime carbogyration) levels of atmospheric $\mathrm{CO}_{2}$. Vital gas exchange in life can now be modeled by nesting the oxygyre and carbogyre in a gyrequation. Given $\mathrm{G}_{\mathrm{I}}$,

$$
\text { ( })=\left(\mathrm{O}^{2 \mathrm{e}} \leftrightarrows[\mathrm{O}]+2 \odot\right)
$$

The oxyon can be nested in the primary carbogyre thusly:

$$
\mathrm{C}^{3 \mathrm{O}} \leftrightarrows \mathrm{C}^{2 \mathrm{O}}+\left(\mathrm{O}^{2 \mathrm{e}} \leftrightarrows[\mathrm{O}]+2 \odot\right)
$$

which models the attractorepulsive relationship between $\mathrm{O}_{2}$ and $\mathrm{CO}_{2}$ (both in bold) found in many living systems:

$$
2 \mathrm{H}_{2} \mathrm{CO}_{3} \leftrightarrows 2 \mathbf{C O}_{2}+\left(2 \mathrm{H}_{2} \mathrm{O} \leftrightarrows \mathbf{O}_{2}+2 \mathrm{H}_{2}\right)
$$

This theoretical formula indicates an early evolutionary emergence for respiratory gas exchange that exists in humans, animals, and plants; this solution is well beyond other ideas [230,231]. A variation on this nested arrangement of these two gyrosystems is found in Figure 3e.

Acid-base homeostasis. The proper balance of acids and bases is necessary in cells, in blood [232], and in the Earth's oceans [233]. Yet, there is no standard model for how acid-base homeostasis evolved. By adding $\mathrm{H}^{+}$, the hydron and $\mathrm{HCO}_{3}{ }^{-}$, bicarbonate, to the reaction above, I get:

$$
\mathrm{H}^{+}+\mathrm{HCO}_{3}^{-} \leftrightarrows \mathrm{H}_{2} \mathrm{CO}_{3} \leftrightarrows \mathrm{CO}_{2}+\mathrm{H}_{2} \mathrm{O}
$$

From this known chemical reaction, I now model the thermodynamic relationships of the photon, electron, oxyon, and carbyon (quantized particle; Figure 2c (iv)), written out to accommodate all of gyrosystems in the fractalized gyrequation:

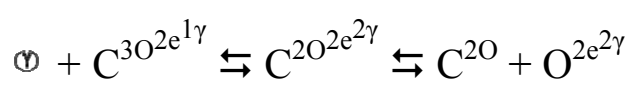

Thus, this theoretical framework neatly dispatches and unifies the microcosmic and macrocosmic origin of acid-base homeostasis.

Carbohydrates. One chemosynthetic step needed for the origin of the living cell is carbohydrate production [234,235]. While it is true that photosynthesis generates a prominent carbohydrate, glucose, several features of this photochemical process have yet to be theoretically clarified [236,237]. Given 
the structural constraints of the secondary majorgyre, in the secondary carbogyre (Figure 2c (ii)), $\left[\mathrm{C}^{\mathrm{O}}\right]$ is the gyrobasal IEM unit with potential to polymerize. Note that the oxyon is the gyrolink in the carbonexus $\left(\mathrm{G}_{\mathrm{X}}\right)$, but is, in fact, orbiting carbon atoms that are gyromodules. Remember that

$$
\left[\mathrm{C}^{\mathrm{O}}\right]=\mathrm{C}^{\mathrm{O}}, \mathrm{C}^{\mathrm{O}} \mathrm{C}^{\mathrm{O}}, \mathrm{C}^{\mathrm{O}} \mathrm{C}^{\mathrm{O}} \mathrm{C}^{\mathrm{O}} \ldots
$$

such that $\left[\mathrm{C}^{\mathrm{O}}\right]_{1}$ is $\mathrm{CH}_{2} \mathrm{O}$ - formaldehyde, the most volatile, ubiquitous, and simplest aldehyde that is the unit component of organic polymers [238-240]. $\left[\mathrm{C}^{\mathrm{O}}\right]_{2}$ is $\mathrm{C}_{2} \mathrm{H}_{4} \mathrm{O}_{2}$ (glycoaldehyde, an important prebiotic chemical [241]); $\left[\mathrm{C}^{\mathrm{O}}\right]_{3}$ is $\mathrm{C}_{3} \mathrm{H}_{6} \mathrm{O}_{3}$ (trioses, e.g., glyceradehyde, which has been suggested to impart chirality to biomolecules [242]; also models pyruvic acid, a hydrothermally reactive compound [243] and the energy source for the citric acid cycle under oxygenating conditions [244]); $\left[\mathrm{C}^{\mathrm{O}}\right]_{5}$ is $\mathrm{C}_{5} \mathrm{H}_{10} \mathrm{O}_{5}$ (pentoses, e.g., ribose, the nucleotide sugar [245,246]); and $\left[\mathrm{C}^{\mathrm{O}}\right]_{6}$ is $\mathrm{C}_{6} \mathrm{H}_{12} \mathrm{O}_{6}$ (hexoses, e.g., glucose and galactose, both notable biomolecules [247,248]). The secondary carbogyre models dehydration of any carbonexus as dioxyon attraction:

$$
\mathrm{C}^{3 \mathrm{O}} \rightarrow\left[\mathrm{C}^{\mathrm{O}}\right]+2 \bigcirc
$$

hydration is modeled as dioxyon repulsion:

$$
\left[\mathrm{C}^{\mathrm{O}}\right]+2 \bigcirc \rightarrow \mathrm{C}^{3 \mathrm{O}}
$$

This ordering of carbonexuses - the theoretical neologism for carbohydrates - is consistent with ideas that formaldehyde and glycoaldehyde are the starting points for carbohydrate metabolism [249]. Further validating the theoretical positioning of formaldehyde, compressing (but not balancing) the primary and secondary carbogyres, I have

$$
\mathrm{C}^{2 \mathrm{O}}+\mathrm{O} \leftrightarrows\left[\mathrm{C}^{\mathrm{O}}\right]+2 \bigcirc
$$

which, given the representational variability of the quantum, the left and right side (Os model the primary and tertiary oxygyre, respectively, exactly fits the following well known reaction in the longterm carbon cycle:

$$
\mathrm{CO}_{2}+\mathrm{H}_{2} \mathrm{O} \leftrightarrows \mathrm{CH}_{2} \mathrm{O}+\mathrm{O}_{2}[250]
$$

Photosynthesis. Photosynthesis is fundamental not only for plant life, but for all life on Earth. The current ideas about the origin and evolution of photosynthesis come from a mosaic of data from biochemistry, biophysics, bioinformatics and physiology [251-254], but these have not coalesced into a general theory. Multimerizing $(n=6)$ the prior gyrequation, I have,

$$
\begin{aligned}
& \left(\mathrm{CO}_{2}+\mathrm{H}_{2} \mathrm{O} \leftrightarrows \mathrm{CH}_{2} \mathrm{O}+\mathrm{O}_{2}\right)_{6} \text {, or } \\
& 6 \mathrm{CO}_{2}+6 \mathrm{H}_{2} \mathrm{O} \leftrightarrows \mathrm{C}_{6} \mathrm{H}_{12} \mathrm{O}_{6}+6 \mathrm{O}_{2}
\end{aligned}
$$

the photosynthesis reaction. The nesting of the photon within the electrogyre, and the electron within the oxygyre, and the oxyon within the carbogyre thus reveals a new algorithm for photosynthetic energy transfer.

Organic cycles. There are several organic cycles throughout the natural world whose origins have remained a mystery. These are now economically fit onto the carbogyre frame. On a cellular scale, the core details of several fundamental processes are modeled as oscillating carbogyres with varying carbon number and form (singularity excluded from the following bidirectional equations): 


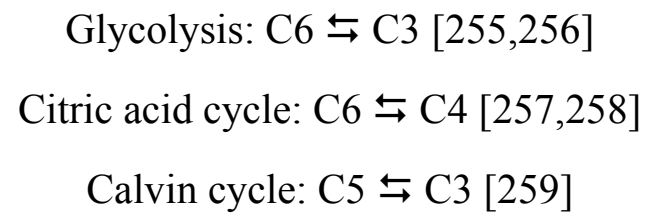

This theory is thus consistent with prior ideas of the citric acid cycle being self-organizing [260]. On a planetary scale, the biogeochemical carbon cycle - the cycling of carbon through the air, oceans, soil, organisms, and sediments [261] — is a macrocarbogyre. One complete cycle for an individual carbyon in this gyrosystem is a carbocycle.

Sugar Homochirality. In 1860, Pasteur proposed an explanation for why sugars are asymmetric (D, right-handed) in living systems, called "chirality" by Lord Kelvin [262]. Despite many ideas for the origin of chemical chirality, there has been no satisfactory explanation. This theoretical framework shows that carbonexuses are only the $\mathrm{D}$ enantiomer because the oxygyre is dextral (Figure $2 \mathrm{~b}$ and Table 2) and, based upon $\mathrm{G}_{\mathrm{XII}}$, the subgyre exerts the formative, directional, vortical force on the matter in primary and secondary majorgyres of the focagyre. Notably, this model is consistent with the empirical observation that vortices induce chiral selection [263].

Higher-order Carbohydrate Structures. Carbonexuses have potential to form higher-order structures such as multimers (e.g., sucrose: $\mathrm{C}_{12} \mathrm{H}_{22} \mathrm{O}_{11}$ ), ramified polymers as in oligosaccharides and polysaccharides (e.g., starch, glycogen, cellulose, and chitin) and also empirically defined helices and matrices theoretically termed carbohelices (Figure 4c; [264-266] — predicted to be left-handed in living systems because sugars are right-handed $\left(\mathrm{G}_{\mathrm{XII}-3}\right)$ - and carbomatrices, respectively. As starch complexifies, it oscillates between helical and latticed states [214]. Because both simple and complex carbohydrates are modeled by the adaptive and responsive carbogyre, this explains the mysterious property of the "feeling" of recognition saccharides [267].

Hydrocarbons. There are two competing theories to explain the origin of hydrocarbons on Earth and its relationship to life. The first, the mainstream biogenic theory, posits that hydrocarbons emerge as a natural result of cellular decomposition and biodegradation of buried organic matter [268]. Given the forcefulness with which this theory has been promoted, subterranean hydrocarbons and petroleum products are called by the moniker "fossil fuels." The second, called abiogenic theory, suggests that organic matter emerges deep within the Earth, partially or largely independent of biodegradation [269-271]. Despite much inquisition [272], there has not been a satisfactory resolution of the biotic/abiotic debate. Can the carbogyre arbitrate this scholarly dispute?

In the tertiary carbogyre (Figure 2c (iii)), the trioxyon (3@; gyradaptor) cycles on and off the carbon atom. In the absence of thermodynamic support of the oxyon, the gyrobase carbon atom establishes thermodynamic relationships the electrogyre $\left(\mathrm{G}_{\mathrm{XI}}\right)$. Hence, in one theoretical incarnation, the gyrobase of the tertiary carbogyre models hydrocarbons as carbonexuses where

$$
[\mathrm{C}]_{\mathrm{n}}=\mathrm{C}^{\mathrm{e}}, \mathrm{C}^{\mathrm{e}} \mathrm{C}^{\mathrm{e}}, \mathrm{C}^{\mathrm{e}} \mathrm{C}^{\mathrm{e}} \mathrm{C}^{\mathrm{e}} \text {, and } \mathrm{C}^{\mathrm{e}} \mathrm{C}^{\mathrm{e}} \mathrm{C}^{\mathrm{e}} \mathrm{C}^{\mathrm{e}} \mathrm{C}^{\mathrm{e}} \mathrm{C}^{\mathrm{e}} \ldots
$$

$\left[\mathrm{C}_{1}\right.$ is $\mathrm{CH}_{4}$ (methane), a crucial and volatile natural gas that has biotic and abiotic importance $[273,274]$. Other important hydrocarbons such as ethane, $\left([\mathrm{C}]_{2}\right.$ is $\left.\mathrm{C}_{2} \mathrm{H}_{6}\right)$, propane $\left([\mathrm{C}]_{3}\right.$ is $\left.\mathrm{C}_{3} \mathrm{H}_{8}\right)$, butane $\left([\mathrm{C}]_{4}\right.$ is $\mathrm{C}_{4} \mathrm{H}_{10}$ ), and so on [275] fit here.

The tertiary carbogyre permits thermodynamic relationships with all chemical elements [276-278]. Some of these organic chemicals are biometabolized by a variety of microorganisms $[279,280]$ and 
many if not most are found in petroleum [281]. The attractive force of trioxyon models oil formation during planetary evolution:

$$
\mathrm{C}^{3 \mathrm{O}} \rightarrow[\mathrm{C}]+3 \mathrm{O}
$$

the countervailing repulsive force models its breakdown:

$$
[\mathrm{C}]+3 \bigcirc \rightarrow \mathrm{C}^{3 \mathrm{O}}
$$

The validity of the carbogyre is further supported by equating the primary and tertiary carbogyres:

$$
\mathrm{C}^{2 \mathrm{O}}+\bigcirc \leftrightarrows[\mathrm{C}]+3 \bigcirc
$$

Compressing and balancing gives

$$
\mathrm{C}^{2 \mathrm{O}} \leftrightarrows[\mathrm{C}]+2 \bigcirc
$$

which, in acknowledging that $\mathrm{H}_{2} \mathrm{O}$ ( (O) was removed from the left-side of the gyrequation during compression, is consistent with the established, if overly simplified relationship:

$$
\mathrm{CO}_{2} \leftrightarrows \text { hydrocarbon }+\mathrm{O}_{2}
$$

a chemical reaction commonly found throughout the climatological, environmental, and energy literature [282-284]. Taken together, then, a macrocarbogyre models the deposition and metabolism of geophysical petroleum in the mantle and crust. As positioned here, the origin of hydrocarbons occurs spatiotemporally prior to the emergence of the living cell.

Immiscibility. The tertiary carbogyre provides an explanation for immiscibility - in this case the inhomogeneity of water and oil [285] — a necessity for the compartmentalized cell to exist. As revealed by the tertiary carbogyre, hydrocarbons harbor no oxyon. Rather, the trioxyon is found exclusively in the singularity, where it exerts a powerful repulsive force to collapse the gyrosystem from its most unstable, high energy state to its relativistically most stable, low energy state. The hydrophobic (water-fearing) relationship between and oil droplet (carbosphere) and water (oxyon) is thus due to this modeled repulsion. From this relationship I have an evolutionary consistent frame for modeling the origin of simple emulsions and non-phosphate membranes [286].

Alcohol. There is no extant general theory for the evolutionary origin of alcohols, although there are predictions regarding chemical constraints for their origin [287]. Here, modeling alcohols requires compression of the secondary and tertiary carbogyres, ultimately revealing the following gyrequation:

$$
\left[\mathrm{C}^{\mathrm{O}}\right] \leftrightarrows[\mathrm{C}]+\mathrm{O}
$$

Given the inverse particle-quantum relationship $\left(\mathrm{G}_{\mathrm{III}}, \mathrm{G}_{\mathrm{III}-1}\right)$, the oxyon in the unit $\left[\mathrm{C}^{\mathrm{O}}\right]$ can represent any primary, secondary, or tertiary oxygyre or combination thereof [288].

This variability amongst the majorgyre frame and subsumed gyrosystems yields an inordinate number of permutations to the individual units in the polymeric form, for example: $\left[\mathrm{C}^{\mathrm{O}}\right]_{1}$ models $\mathrm{C}^{\mathrm{O}^{3 \mathrm{e}}}$, which is $\mathrm{CH}_{3} \mathrm{O}$, or methanol; $[\mathrm{C}]\left[\mathrm{C}^{\mathrm{O}}\right]$ models $\mathrm{C}^{3 \mathrm{e}} \mathrm{C}^{\mathrm{O}}$, which is $\mathrm{C}_{2} \mathrm{H}_{6} \mathrm{O}$, or ethanol; $\left[\mathrm{C}^{\mathrm{O}}\right]_{3}$ models $\mathrm{C}^{\mathrm{O}^{3 \mathrm{e}}} \mathrm{C}^{\mathrm{O}^{2 \mathrm{e}}} \mathrm{C}^{\mathrm{O}^{3 \mathrm{e}}}$, which is $\mathrm{C}_{3} \mathrm{H}_{8} \mathrm{O}_{3}$, or glycerol; and so on. Glycerol is a fundamental unit of any phospholipid and thus its origin is of great interest to biopoesists [289].

Fatty acid. The current chemical model for cellular fatty acid synthesis involves acetyl-coA and malonyl-coA precursors and proteins called fatty acid synthases [290,291]. The fundamental problem 
with this model from an evolutionary standpoint is that metabolic processes undergird the formation of RNA, which is required for creating any protein. An alternative pathway focused on chemical origins, where primitive fatty acid synthesis is dependent upon a glycoaldehyde substrate, has been proposed [292]. Given these differing views, I applied the $1 \% 3^{\circ}$ alternacarbogyre to modeling simple and complex fatty acids. For example, the polymer $\mathrm{C}^{2 \mathrm{O}}[\mathrm{C}]_{\mathrm{n}}$ is any fatty acid-where $\mathrm{C}^{2 \mathrm{O}}$ models a carboxyl "head" group $\left(\mathrm{COOH}\right.$; rather than carbon dioxide) and, for the $[\mathrm{C}]_{\mathrm{n}}$ "tail," for example, the $[\mathrm{C}]_{1}$ moiety is $-\mathrm{CH}_{3}$, acetic acid, an important molecule for the origin of life [293,294]; [C] $]_{2}$ is $-\mathrm{CH}_{3} \mathrm{CH}_{2}$, propionic acid, a nutritionally relevant fatty acid [295]; and [C] $]_{13}$ is $-\left(\mathrm{CH}_{2}\right)_{12} \mathrm{CH}_{3}$ is myristic acid, a regulatory fatty acid [296,297]. Any other saturated or unsaturated fatty acid [298,299] in distinct microorganisms [300,301] can be similarly modeled. Given the emergence of glycerol and the nature of fractal Matrioshkagyre sets, any mono-, di-, and triglyceride [302] can likewise be positioned here.

Asteroids and Comets. The Solar System has an asteroid belt between Mars and Jupiter and another, the Kuiper belt, beyond Neptune's orbit [303,304]. It has been argued that these and other asteroids are remnants of Solar System genesis - collisional break-up of large parental bodies [305]. One other class of prominent bodies is comets, whose origins are thought to be found in the Kuiper belt or in the Oort Cloud, a hypothetical cloud of icy bodies at the edge of the Solar System [306,307]. Because the electrogyre and oxygyre have cosmic applications - modeling the origin of planets (3.1) and moons (3.2), respectively - I thus applied the carbogyre to mysterious origins of asteroids and comets. Since asteroids and comets are carbonaceous and largely icy [308,309] — a comet is, by definition, 85\% icethis is consistent with the oxyon singularity at the core of the carbogyre. Moreover, the origin, evolution, and organization of the carbonates and carbonatites that comprise meteorites [310] and the Earth [311] are accounted for by attractorepulsion of the gyrapex of the carbogyre by the electrogyre. The gyrobases of the secondary and tertiary carbogyre models polyols as well, like those found in the Murchison meteorite [312]. Thus, the carbogyre is a broad-ranging model for understanding the physical properties and behavior of asteroids, comets, meteors, and other related celestial objects [313].

From Organic Chemistry to Phosphochemistry. How the living cell and planet Earth both are capable of producing and perennially reproducing their exquisite carbon chemistry and biochemistry in exactitude is modeled as carbognosis - universal learning of organic compounds in response to adaptational cues derived from electron and oxyon flow — and carbomnemesis - retention and retrieval of the ordering. Notably, these phenomena support the organic nature of the Earth found in the Gaia hypothesis [314-316].

The nesting of the photon within the electrogyre, the electron in the oxygyre, the electron in the carbogyre, and the oxyon in the carbogyre reveals how carbohydrates and hydrocarbons store energy and information that performs - and can be extracted to perform - specific physical, biophysical, and geophysical work. As found in almost all critical biometabolic pathways in the three kingdoms of life, the cycling of organic matter sustains and adapts the phosphochemical systems of life.

\subsection{Phosphomembranes}

All living cells have membranes composed of phospholipids that are necessary for compartmentalization of biometabolic processes [317-319]. All living cells use the high-energy 
phosphate bond for mobilizing energy from one molecule to another [320]. Phosphorus is also thought to be geochemically important to the evolution of early Earth [321]. How and why phosphorus has such a prominent role in both the structure and function of life is an unsolved matter.

In the search for the origin of life, paleobiologists have suggested that finding distinct membranous architectures in ancient rock layers is a key hint regarding the existence of life during geological epochs [322,323]. It is unclear whether the paleobiological findings reflect imprints of membranous compartments (coacervates [324], lipid vesicles [325], protocells [326]) filled with a metabolic soup, although certain additional biological signatures are also used to claim what is and is not a true fossilized cell. A scientific theory of life should inform whether membranous cavities emerged prior to dividing cells. In addition to addressing this matter, the phosphogyre models the origin and evolution of the high-energy phosphate bond that all living organisms on Earth use for storing and mobilizing chemical energy.

From the phosphogyre onward, the reader should appreciate that the gyromodel-defined quanta are polymers, systems, and molecular aggregates; this symbolic representation contrasts the quantized electrons, elements, and chemical molecules as outlined in the electrogyre, oxygyre, and carbogyre. For theoretical thrift and consistency, the phosphogyre does not symbolically disclose the oxyon, electron, and photon even though they are subsumed by it $\left(\mathrm{G}_{\mathrm{VIII}}\right)$.

Phosphoenolpyruvate. In reaction chemistry and biochemistry, the Gibbs free energy $(\Delta \mathrm{G})$ is a value that represents the thermodynamic potential to do work [327,328]; the lower the value, the greater potential. The molecule with the highest chemical bond energy in life $(\Delta \mathrm{G}=-61.9 \mathrm{~kJ} / \mathrm{mol})$ is the versatile small molecule phosphoenolpyruvate (PEP) [329]. In the bacterial cell, PEP is the energy source for the phosphotransferase system [330]. So: How did PEP emerge in the evolution of life? Considering that the primary phosphogyre (Figure $2 \mathrm{~d}(i)$ ) is

$$
\mathrm{P}^{3 \mathrm{C}} \leftrightarrows \mathrm{P}^{2 \mathrm{C}}+\mathrm{C}
$$

this fits the chemical reaction

$$
\mathrm{C}_{3} \mathrm{H}_{5} \mathrm{O}_{6} \mathrm{P} \leftrightarrows \mathrm{C}_{2} \mathrm{H}_{3} \mathrm{O}_{5} \mathrm{P}+\mathrm{CH}_{2} \mathrm{O}
$$

which shows interconversion between PEP $\left(\mathrm{C}_{3} \mathrm{H}_{5} \mathrm{O}_{6} \mathrm{P}\right)$ and acetyl phosphate $\left(\mathrm{AcP} ; \mathrm{C}_{2} \mathrm{H}_{3} \mathrm{O}_{5} \mathrm{P}\right)$ and formaldehyde $\left(\mathrm{CH}_{2} \mathrm{O}\right)$, the gyradaptive singularity - the carbyon that is rapidly polymerized $\left(\left[\mathrm{C}^{\mathrm{O}}\right]\right)$ in the gyrobase of the secondary carbogyre. Whereas AcP is a phosphate donor molecule with central roles in bacterial biosynthetic and nutrient sensing pathways [331,332], PEP is the penultimate component in glycolysis and the second component in gluconeogenesis [333]. Further evidence of the relationship of PEP and AcP to the oxygyre and electrogyre is found in the following oxidation-reduction reaction (a variant on the pyruvate oxidase reaction that oxidizes pyruvate $\left(\mathrm{C}_{3} \mathrm{H}_{3} \mathrm{O}_{3}{ }^{-}\right)$to acetate $\left(\mathrm{C}_{2} \mathrm{H}_{3} \mathrm{O}_{2}{ }^{-}\right)$and $\left.\mathrm{CO}_{2}[334]\right)$ :

$$
\text { pyruvate }+\mathrm{P}_{\mathrm{i}}+\mathrm{O}_{2} \leftrightarrows \mathrm{AcP}+\mathrm{CO}_{2}+\mathrm{H}_{2} \mathrm{O}_{2}
$$

Here, $\mathrm{P}_{\mathrm{i}}$ is inorganic phosphate $\left(\mathrm{HPO}_{4}{ }^{2-}\right)$.

$\mathrm{PEP}$ is also involved in another very important photosynthetic process in plants called $\mathrm{C} 4$ carbon fixation [335]. I can modify the longhand reaction

$$
\mathrm{PEP}+\mathrm{CO}_{2}+\mathrm{H}_{2} \mathrm{O} \rightarrow \text { oxaloacetic acid }+\mathrm{P}_{\mathrm{i}}
$$


to

$$
\mathrm{PEP}+\mathrm{H}_{2} \mathrm{CO}_{3} \rightarrow \mathrm{OAA} \sim \mathrm{P}_{\mathrm{i}}
$$

showing both the unstable carbonic acid and unstable transition state $(\sim)$ phosphate molecule. OAA has the chemical notation of $\mathrm{C}_{4} \mathrm{H}_{4} \mathrm{O}_{5}{ }^{2-}$, the equation balances and can be written in the bidirectional, inverted gyrequation shorthand:

$$
\mathrm{P}^{4 \mathrm{C}} \leftrightarrows \mathrm{P}^{3 \mathrm{C}}+\mathrm{C}
$$

This crucial step in C4 fixation is thus an alternagyre that oscillates between 4 carbyon (excited; gyrapex) and 3 carbyon (ground; gyrobase) states. I have depicted the carbonic acid-as-singularity in Figure $3 \mathrm{~d}$ and have put the electrogyre, oxygyre, carbogyre, and this phosphogyre into a Matrioshkagyre (Figure 3e). Notably, OAA is a component in the citric acid cycle [336], and, being composed of $\mathrm{C}, \mathrm{H}$, and $\mathrm{O}$, is effectively modeled in the secondary carbogyre. The carbyon-phosphogyre nesting is thus theoretically compatible with the empirical evidence.

Theory thus shows that, like the emergence of water (3.2) and carbon dioxide (3.3) before, phosphorous compounds emerge from the expansion of the universe from within the electrogyre, into and through the oxgyre and carbogyre, and forming a gyrosystem with relativistically lower exergy, opposing chirality, and evolutionary novelty $\left(G_{X I I I}, G_{V I}, G_{I X}\right)$. Together, this expansive force, the inherent creatodestructive nature of the gyre, and the fact that $\Delta \mathrm{G}$ is derived from the energy-rich photons housed in the electrons themselves (positioning PEP proximal to the gyradaptive singularity), cumulatively explain the origin of the high-energy phosphate bond.

Phospholipids. The biochemical and molecular structure, function, and regulation of cellular phospholipids is well known [337,338]. However, the field lacks a unifying framework. Here, the primary phosphogyre models the most basic of all phospholipids, where the gyrapical $\mathrm{P}^{3 \mathrm{C}}$ models 3-carbon (3C) glycerol "backbone" with a diglyceride "tail" and a 1-orthophosphate "head" group (P; additional carbyons, oxyons, and electrons are excluded for theoretical tidiness; note the triquantal organization). The cycling carbyon (C); gyradaptor) models fatty acids (3.3) which, given $\mathrm{G}_{\mathrm{I}}$, exerts the thermodynamic attractive force, or breakdown of the two-tail $\left(\mathrm{P}^{3 \mathrm{C}}\right)$ phospholipid to a one-tail $\left(\mathrm{P}^{2 \mathrm{C}}\right)$ state:

$$
\mathrm{P}^{3 \mathrm{C}} \rightarrow \mathrm{P}^{2 \mathrm{C}}+\mathrm{C}
$$

and the countervailing repulsive force to construct the two-tail state:

$$
\mathrm{P}^{2 \mathrm{C}}+\left(\mathrm{C} \rightarrow \mathrm{P}^{3 \mathrm{C}}\right.
$$

Based upon this model, fatty acid uptake is biophysically "easy [339]." Lexically, one cycle of the carbyon particle through the phosphogyre is called a phosphocycle.

Because the primary phosphogyre expands from the singularity omnidirectionally outwards, monolayer single-tailed phospholipids form micelles (see below for bilayered structures), spherical structures called here phosphospheres [340,341].

In the secondary phosphogyre (Figure $2 \mathrm{~d}(\mathrm{ii})$ ), two carbyons cycle through the singularity:

$$
\mathrm{P}^{3 \mathrm{C}} \leftrightarrows\left[\mathrm{P}^{\mathrm{C}}\right]+2 \subseteq
$$


causing the gyrosystem dIEM to exist as either $\left(\mathrm{G}_{\mathrm{V}}\right)$ the high energy, learning state (the two-tailed phospholipid is a phosphognose) or the low energy memory state (phosphomneme) with the potential to polymerize ([ $\left.\mathrm{P}^{\mathrm{C}}\right]$; gyrobase). In the polymer, called a phosphonexus, $\mathrm{C}$ is retained as the gyrolink $\left(\mathrm{G}_{\mathrm{X}}\right)$ that facilitates polymerization with $\mathrm{P}_{\mathrm{i}}$ gyromodules:

$$
\left[\mathrm{P}^{\mathrm{C}}\right]=\mathrm{P}^{\mathrm{C}}, \mathrm{P}^{\mathrm{C}} \mathrm{P}^{\mathrm{C}}, \mathrm{P}^{\mathrm{C}} \mathrm{P}^{\mathrm{C}} \mathrm{P}^{\mathrm{C}}, \mathrm{P}^{\mathrm{C}} \mathrm{P}^{\mathrm{C}} \mathrm{P}^{\mathrm{C}} \mathrm{P}^{\mathrm{C}} \mathrm{P}^{\mathrm{C}} \ldots
$$

I want to call attention to what theory predicts: the phospholipid head group separates from its two-tail group, leaving an "untailed" head group consisting of the C1 of glycerol and the orthophosphate and a "headless" $\mathrm{C} 2$ and $\mathrm{C} 3$ of glycerol with the tail group of acyl chains. In a phosphonexus, one carbyon gyrolinks two flanking phosphons (quantized particle (Figure 3d (iv)) known as two monoester bonds (oxygyre). Membrane fluidity - which has been understood largely from the fluid mosaic model [342,343] — can now be clarified as phosphonexus structure and metabolism, or oscillation between the anabolic state generated by the attractive force of the dicarbyon,

$$
\mathrm{P}^{3 \mathrm{C}} \rightarrow\left[\mathrm{P}^{\mathrm{C}}\right]+2 \mathrm{C}
$$

and the catabolic state, repulsion by the dicarbyon that regenerates the phospholipid,

$$
\left[\mathrm{P}^{\mathrm{C}}\right]+2 \mathrm{C} \rightarrow \mathrm{P}^{3 \mathrm{C}}
$$

The innate adaptability of the phosphogyre explains homeoviscous adaptation [344]. Fluidity is also better understood as the spacetime path of the carbyon in its orbit around the phosphon in the phosphonexus. The unitary $\mathrm{P}^{\mathrm{C}}$ accommodates at least two different chemistries: $(i)$ methyl phosphate $(\mathrm{MeP})$, a phosphoryl transfer molecule with a very fast rate of hydrolysis (oxygyre attraction (3.2); [345,346]) and (ii) carboxyl phosphate, which is a short-lived biochemical intermediate [347]. Oxygyre repulsion would model condensation and hence polymerization $\left(\left[\mathrm{P}^{\mathrm{C}}\right]\right)$. Longer phosphonexuses are predicted to be in a levoral organization (Table 2) called phosphohelices that toggle between the two chiralities $\left(\mathrm{G}_{\mathrm{XII}-3}\right)$ as they structurally complexify. These phosphonexuses and phosphohelices verifiably assemble into phosphomatrices, a layered architecture that, in effect, "floats" on top of the headless dicarbyon.

With this model, I can now explain several enigmatic features of phosphomembranes. First, the floating phenomenon models what has been described in the literature as lipid rafts [348], as surface areas move as an ensemble. Second, given the repulsive nature of the oxyon on the tertiary carbogyre (3.3), the acyl chains align and appose, modeled as the antiparallel flow of one phosphogyre along another - one phosphogyre exerts a countervailing force against the other, providing instrinsic and extrinsic balancing, i.e. "cross talk" between the two layers [349]. This antiparallel structure represents the phospholipid bilayer that is found in all cell membranes and in organelles (e.g., Golgi apparatus, endoplasmic reticulum, peroxisome, vacuole [350-356]). Third, because an individual acyl chain can be metabolized by the singularity in either one of the two antiparallel carbogyres, this explains lipid diffusion and spontaneous fatty acid flip-flop [357,358]. Fourth, the inherent adaption of the phosphogyre explains the balance of phosphate chemistry within and without of a membrane compartment, called phosphate homeostasis $[359,360]$. Fifth, phase shifting to and from the membrane solid state [361] is explained by phosphogyre arrest and release, respectively — changing photon (energy) 
flow through the electrogyre, oxygyre, and carbogyre controls phosphon cycling (phosphocycling) between gyrostates.

If $\mathrm{P}^{3 \mathrm{C}}$ is modeled to be the two-tailed phospholipid and $\left[\mathrm{P}^{\mathrm{C}}\right]$ is phosphonexus, then what is the gyradaptive 2(C)? As fit to the gyromodel, 2 (C) corresponds to cholesterol [362], members of a family of sterol molecules that are involved in numerous signaling cascades [363], vitamins [364], coenzymes [365,366], flavonoids [367], and tocopherols [368]. These compounds emerge in the carbogyre and exert strong thermodynamic forces as the gyradaptive singularity of the phosphogyre.

Polyphosphate. All kingdoms of life have inorganic polyphosphates that can range in length from a few orthophosphates to several hundred long. These polyphosphates have been implicated in a variety of essential biological phenomena including, but not limited to energy storage, biofilms formation, stress-induced gene regulation, cell motility, virulence, cellular proliferation, differentiation, and development [369,370]. For these and other reasons, Kornberg asserted that polyphosphate is of genuine theoretical interest as a prebiotic precursor to RNA, protein, and DNA [371].

I have applied the empirical evidence about polyphosphates to one of the majorgyres of the gyromodel, the tertiary phosphogyre (Figure $2 \mathrm{~d}(\mathrm{iii})$ ). This gyrosystem shows the extreme cycling of the tricarbyon (3 (C); gyradaptor) which models a monoglyceride [372,373], diacylglycerol—an established second messenger signaling lipid [374]— or any of a number of trigylcerides [375,376]. The attractive force of the tricarbyon on the phosphogyre elicits the formation of a phosphonexus:

$$
\mathrm{P}^{3 \mathrm{C}} \rightarrow[\mathrm{P}]+3 \mathrm{C}
$$

Given $\mathrm{G}_{\mathrm{XI}}$, the gyrobasal $[\mathrm{P}]$ has a oxyon gyrolink and models: orthophosphoric acid $\left([\mathrm{P}]_{1}=\mathrm{P}^{\mathrm{O}}\right.$; $\mathrm{H}_{3} \mathrm{PO}_{4}$ ), the hallmark of all protein signal transduction cascades [377]; pyrophosphoric acid $\left([\mathrm{P}]_{2}=\mathrm{P}^{\mathrm{O}} \mathrm{P}^{\mathrm{O}} ; \mathrm{H}_{4} \mathrm{P}_{2} \mathrm{O}_{7}\right)$, found in all nucleic acid polymerization reactions (3.5 and 3.7) and numerous intracellular and extracellular processes [378,379]; triphosphoric acid $\left([\mathrm{P}]_{3}=\mathrm{P}^{\mathrm{O}} \mathrm{P}^{\mathrm{O}} \mathrm{P}^{\mathrm{O}} ; \mathrm{H}_{5} \mathrm{P}_{3} \mathrm{O}_{10}\right)$, one of three major parts of the nucleotide triphosphates (3.5); and polyphosphoric acid $\left([\mathrm{P}]_{\mathrm{n}}=\mathrm{P}^{\mathrm{O}} \mathrm{P}^{\mathrm{O}} \mathrm{P}^{\mathrm{O}} \mathrm{P}^{\mathrm{O}} \mathrm{P}^{\mathrm{O}} \ldots\right)$. Notably, polyphosphates assemble into helices (Figure 4d; [215,380]). The catabolism of these polymers is modeled vectorially as:

$$
[\mathrm{P}]+3 \mathrm{C} \rightarrow \mathrm{P}^{3 \mathrm{C}}
$$

where tricarbyon generates the PEP or phosopholipid molecule. In this regard, another notable reaction can be neatly fit onto the tertiary phosphogyre:

$$
\mathrm{PEP} \leftrightarrows \mathrm{P}_{\mathrm{i}}+\text { pyruvate }
$$

The relationship between the three majorgyres can be equated through the shared gyrapex,

$$
\mathrm{P}^{2 \mathrm{C}}+\left(\mathrm{C} \leftrightarrows\left[\mathrm{P}^{\mathrm{C}}\right]+2 @ \leftrightarrows[\mathrm{P}]+3 @\right.
$$

balancing, I have:

$$
\mathrm{P}^{2 \mathrm{C}} \leftrightarrows\left[\mathrm{P}^{\mathrm{C}}\right]+(\mathrm{C} \leftrightarrows[\mathrm{P}]+2 @
$$

This gyrequation provides a parsed relationship between phosphochemical energy storage and lipid signaling pathways in all cells, which, written longhand, can be:

one-tailed phospholipids $\leftrightarrows$ phosphonexuses + fatty acids $\leftrightarrows$ polyphosphates + sterols. 
Other phosphorous compounds that are important to the evolution of the early Earth are modeled here [381-384].

Biogeochemistry. The phosphorus cycle is one of the major biogeochemical cycles that occurs on Earth and is essential for life [385-387]. Together, the thermodynamic relationships from the electrogyre (3.1) to the oxygyre (3.2), carbogyre (3.3), and phosphogyre capture elemental, inorganic, aqueous, and organic states of the phosphorus cycle. Like all other biogeochemical cycles that are viewed in four dimensions, the phosphorus cycle is a macrophosphogyre.

From Phosphochemistry to Genetic Information. I have shown the phosphogyre to have broad explanatory power. Given that there is no general theory of phosphate biochemistry, the phosphogyre affords unparalleled insight into fundamental characteristics of all life on Earth. Given that all cellular phosphate molecules are modeled to undergo phosphognosis and phosphomnemesis in response to cues from sub- and supervenient gyrosystems, this supports the concept of membrane heredity [388]. Moreover, the phosphogyre helps clarify the omnidirectional phospholipid façade of the cell, membrane-bound organelle inheritance [354], functional symmetry of endomembranes [389], and the establishment and maintenance of its phosphate-dependent signal transduction cascades. Regarding the evolution of life, the nested gyrosystem architecture demonstrates that phospholipid structures formed prior to nucleic acids, proteins, and dividing cells, consistent with hypotheses related to minimum protocell evolution [390] and the lipid world model [391].

At this point, I have a framework that seamlessly integrates phosphate $(\mathrm{P})$ and carbon $(\mathrm{C}$, carbogyre) chemistry, oxygen (O, oxygyre), and hydrogen ( $\mathrm{H}$, primary electrogyre), or CHOP. However, because life is $\sim 98 \%$ CHNOPS, I require models that fit the scientific data regarding biomolecules composed of nitrogen (N) and sulfur (S). In knowing that orthophosphate is a core component of all nucleic acids and being practiced in theoretical RNA biology [392], I modeled the phosphon as the thermodynamic driving force for the emergence of nucleotides and genetic information.

\section{5. $R N A$}

The RNA molecule that transmits genetic information is an essential feature of all life. Current notions for how cells are genetically regulated are derived from Crick's central dogma [393]. This dogmatic model specifies the following: ( i) DNA is the long-term, stable genetic storehouse; (ii) DNA is a template for messenger RNA (mRNA), a short-lived molecular go-between; (iii) mRNA, along with ribosomal RNA (rRNA) and transfer RNA (tRNA), assemble amino acids into polypeptides (proteins). This parsed linear flow of genetic information is

$$
\text { DNA } \rightarrow \text { RNA } \rightarrow \text { protein }
$$

where the arrow between DNA and RNA is the process called transcription and that between RNA and protein is called translation.

Several pieces of evidence and investigators have called this model into question [394-396] and have implied a more primal role for RNA than originally thought. Gilbert was the first to formally promulgate the RNA world hypothesis, in which RNA emerged evolutionarily prior to DNA and protein [397]. Although his radical proposal is compatible with the available data [7-9], there is no genetic theory that includes and validates the hypothesis. On this note, the ribogyre is a theoretical framework for understanding the emergence, adaptation, and metabolism of genetic information. 
Nucleotide triphosphate. How the nucleotide originated is one of the more challenging biosynthetic enigmas [398-401]. Here, I fit the well-known biochemical reaction:

$$
\mathrm{NTP} \leftrightarrows \mathrm{NDP}+\mathrm{P}_{\mathrm{i}}
$$

where NTP and NDP are the nucleotide tri- and diphosphates, respectively, and $\mathrm{P}_{\mathrm{i}}$ is the gyromodule of the tertiary phosphogyre (3.4), onto the primary ribogyre (Figure $2 \mathrm{e}(i)$ ). That reaction, as a gyrequation, is:

$$
\mathrm{R}^{3 \mathrm{P}} \leftrightarrows \mathrm{R}^{2 \mathrm{P}}+\mathrm{P}
$$

$\mathrm{R}$ corresponds to the grouped nucleotide sugar and nitrogenous base and gyradaptive $\mathrm{P} / \mathrm{P}$ is the particle/quantum orthophosphoric acid, the phosphon - the mIEM and singularity of the ribogyre. Given the protean quality of gyromodel symbolism, the gyrapical $\mathrm{R}^{3 \mathrm{P}}$ and gyrobasal $\mathrm{R}^{2 \mathrm{P}}$ can represent any one or all NTPs (adenosine triphosphate (ATP), guanosine triphosphate (GTP), cytidine triphosphate (CTP), and uridine triphosphate (UTP)) and NDPs, respectively.

In the ribogyre, NTP generation is modeled as the repulsive force of the phosphon on an NDP molecule itself:

$$
\mathrm{R}^{2 \mathrm{P}}+\circledast \rightarrow \mathrm{R}^{3 \mathrm{P}}
$$

Conversely, NTP catabolism is modeled as the attractive force of the phosphon:

$$
\mathrm{R}^{3 \mathrm{P}} \rightarrow \mathrm{R}^{2 \mathrm{P}}+\mathrm{P}
$$

The primary ribogyre thus accounts for all NTP/NDP cycles required to establish energy and matter gradients in cell regulation and signal transduction pathways [402,403],

$$
\mathrm{ATP} \leftrightarrows \mathrm{ADP}+\odot
$$

vesicle [404] and cargo [405] transport,

$$
\mathrm{GTP} \leftrightarrows \mathrm{GDP}+\odot
$$

sugar [406,407] synthesis;

$$
\mathrm{UTP} \leftrightarrows \mathrm{UDP}+\odot
$$

and lipid [408] synthesis,

$$
\mathrm{CTP} \leftrightarrows \mathrm{CDP}+\odot
$$

Please note the triquantal feature of the NTP molecule: nitrogenous base (tertiary ribogyre, see below), ribose (secondary carbogyre), and triphosphate (tertiary phosphogyre). Additional carbyonribogyre relationships facilitate modeling molecules called nucleotide sugars [409].

I call attention to four gyraxioms as they relate to this gyrosystem. First, the generation of the ATP in the ribogyre is consistent with the dependence of subsumed gyrosystems $\left(\mathrm{G}_{\mathrm{VIII}}\right)$ and with the chemiosmotic hypothesis [410] — it is thermodynamically dependent upon electron mobilization (electrogyre), in redox reactions (oxygyre), through organic matter (carbogyre), in a phospholipid membrane (phosphogyre), onto water (oxygyre), with deposition of accumulated potential energy into orthophosphate (phosphogyre), on a nucleotide (ribogyre). Second, a nucleotide can exist in either NTP or NDP form but cannot exist in both states at the same time $\left(\mathrm{G}_{\mathrm{V}}\right)$. Third, it is also known that nucleotides are exclusively dextral in life-L-nucleotides are unnatural and cause significant structural 
alterations to nucleic acid structures [411] — but it is not clear why. The chirality of the nucleotide is dextral because the phosphogyre is dextral $\left(\mathrm{G}_{\mathrm{XII}}\right)$. Fourth, the ribogyre, in emerging from the phosphogyre, dictates that ATP is relativistically less exergic but more stable $\left(\mathrm{G}_{\mathrm{XIII}}\right)$ than pyrophosphate and also more evolved [412,413].

Transcription and turnover. The idea that RNA is "transcribed" from-that is, copied or templated from-DNA is deeply engrained in the scientific literature [414-416]. However, the origin of novel small RNA species [417,418], changes to or rearrangements in RNA sequence [419,420], intronic origins [421], and different RNA turnover rates [422] have not been adequately explained by the DNA-centric RNA biogenesis idea. I thus determined how the majorgyre frame could resolve these discrepancies.

The secondary ribogyre (Figure $2 \mathrm{e}(\mathrm{ii})$ ) models the metabolism of RNA:

$$
\mathrm{n} \times \mathrm{NTP} \leftrightarrows \mathrm{RNA}+\mathrm{n} \times \mathrm{P} \sim \mathrm{P}
$$

where $\mathrm{P} \sim \mathrm{P}$ is pyrophosphate and $\mathrm{n}=$ any positive integer. This precisely fits the gyrequation:

$$
\mathrm{R}^{3 \mathrm{P}} \leftrightarrows\left[\mathrm{R}^{\mathrm{P}}\right]+2 \oplus
$$

where, again, $\mathrm{R}^{3 \mathrm{P}}$ is $\mathrm{NTP}$, the shared gyrapex of the majorgyres, $2 \odot$ is pyrophosphate or two orthophosphates, and $\left[\mathrm{R}^{\mathrm{P}}\right]$ is a nucleotide monophosphate (NMP) with the potential to polymerize into RNA, referred to here as a ribonexus. For example,

$$
R^{\mathrm{P}}, \mathrm{R}^{\mathrm{P}} R^{\mathrm{P}}, \mathrm{R}^{\mathrm{P}} R^{\mathrm{P}} R^{\mathrm{P}}, \mathrm{R}^{\mathrm{P}} R^{\mathrm{P}} R^{\mathrm{P}} R^{\mathrm{P}} R^{\mathrm{P}} R^{\mathrm{P}} \ldots
$$

is the same as

mononucleotide, dinucleotide, trinucleotide, and hexanucleotide.

Note that, in the ribonexus, the phosphon $(\mathrm{P})$ is the gyrolink and the mononucleotides $(\mathrm{R})$ are the gyromodules $\left(\mathrm{G}_{\mathrm{X}}\right)$.

Now, with this new understanding, transcription (RNA "expression") is modeled vectorially as diphosphon attraction,

$$
\mathrm{R}^{3 \mathrm{P}} \rightarrow\left[\mathrm{R}^{\mathrm{P}}\right]+2 \odot
$$

and RNA turnover is modeled by the repulsive force of the diphosphon,

$$
\left[\mathrm{R}^{\mathrm{P}}\right]+2 \odot \rightarrow \mathrm{R}^{3 \mathrm{P}}
$$

with ribonexus disassembly into component nucleotides that are restored to the gyrapical state.

The closer the ribonexus is to the gyradaptive diphosphon, the faster it cycles between the two gyrostates; the further from the singularity, the slower it cycles. The secondary ribogyre thus affords a new perspective on the varying RNA half-lives and cellular transcription cycle (ribocycle; [423,424]) where viewing in four dimensions reveals the gyre. I present the diphosphon-as-singularity concept in Figure $3 \mathrm{f}$. Combining, compressing, and reducing the primary and secondary ribogyres yields:

$$
\mathrm{R}^{2 \mathrm{P}} \leftrightarrows\left[\mathrm{R}^{\mathrm{P}}\right]+\oplus
$$

which validates the interconversion of NDPs and NMPs and both confirms and predicts a basic biometabolic relationship between NDPs and RNAs [425,426]. The cycling of the full complement of 
RNAs (transcriptome) in a cell, organism, or species in an ecosystem or planet occurs within a macroribogyre.

RNA Structure and Function. RNAs continually adapt and evolve through a process known as ribognosis, whereby gyrapical NTPs import and integrate information related to the phosphochemical energy state of membrane compartment (phosphon). The gyrobase of the ribogyre, being a ribomneme, stores information about nucleotide content for all RNA classes, especially the three main classes: mRNA [427,428], tRNA [429,430], and rRNA [431,432]; I return to these in 3.6. Similar to other gyronexuses, ribonexuses form higher order structures theoretically defined as ribohelices (e.g., stem-loops [433], hairpins [434]) and ribomatrices (Figure 4e; splicing RNAs [435], rRNA complexed with mRNA and tRNA [436]) that toggle between chiralities as they complexify $\left(\mathrm{G}_{\mathrm{XII}-3}\right)$.

Secondary messengers and cofactors. Given its symbolic depth and intrinsic gyrosystems, $\mathrm{R}^{\mathrm{P}}$ models cyclic AMP (cAMP; [437,438]) and cGMP [439]; $\left[\mathrm{R}^{\mathrm{P}}\right]_{2}$ models dinucleotide molecules (e.g., cyclic di-GMP [440,441], nicotinamide adenine dinucleotide (NAD; [442])). Other nitrogenous cofactors are positioned here $[443,444]$.

Genetic code. The origin of the genetic code is one of the leading problems in evolutionary biology [445] and thus pinning down this problem requires a ground head chancery. Since majorgyres dictate that energy and matter assemble into triquantal (most exergic, least stable), diquantal (intermediate energy and stability), and uniquantal (least exergic, most stable) states (2.4.5), I applied this concept to understand the organization of genetic information. As modeled sans proteins, within the ribonexus, $\left[\mathrm{R}^{\mathrm{P}}\right]_{3}$, the trinucleotide—rather than the mononucleotide as used by polymerases $[446,447]$ is the high-energy triquantal unit that polymerizes, where

$$
\begin{gathered}
3 \mathrm{R}^{3 \mathrm{P}} \leftrightarrows\left[\mathrm{R}^{\mathrm{P}}\right]_{3}+6 \odot \text {, and } \\
{\left[\mathrm{R}^{\mathrm{P}}\right]_{3}=\mathrm{R}_{\xi}{ }^{\mathrm{P}} \mathrm{R}_{\psi}{ }^{\mathrm{P}} \mathrm{R}_{\zeta}{ }^{\mathrm{P}}}
\end{gathered}
$$

and $\mathrm{R}_{\xi}{ }^{\mathrm{P}}$ is first nucleotide of the codon, $\mathrm{R}_{\psi}{ }^{\mathrm{P}}$ is second nucleotide, $\mathrm{R}_{\zeta}{ }^{\mathrm{P}}$ is third nucleotide. The dynamics of this gyrosystem can also be visualized via two gyrequations:

$$
\begin{gathered}
\mathrm{R}_{\xi}{ }^{\mathrm{P}} \mathrm{R}_{\psi}{ }^{\mathrm{P}} \mathrm{R}_{\zeta}{ }^{\mathrm{P}} \leftrightarrows \mathrm{R}_{\xi}{ }^{\mathrm{P}} \mathrm{R}_{\psi}{ }^{\mathrm{P}}+\mathrm{R}_{\zeta}{ }^{\mathrm{P}} \\
\mathrm{R}_{\xi}{ }^{\mathrm{P}} \mathrm{R}_{\psi}{ }^{\mathrm{P}} \mathrm{R}_{\zeta}{ }^{\mathrm{P}} \leftrightarrows \mathrm{R}_{\xi}{ }^{\mathrm{P}}+\mathrm{R}_{\psi}{ }^{\mathrm{P}} \mathrm{R}_{\zeta}{ }^{\mathrm{P}}
\end{gathered}
$$

Although the triribonexus $\left(\mathrm{R}_{\xi}{ }^{\mathrm{P}} \mathrm{R}_{\psi}{ }^{\mathrm{P}} \mathrm{R}_{\zeta}{ }^{\mathrm{P}}\right.$; triquantum) can be modeled in the secondary ribogyre, here, the $\mathrm{R}_{\zeta}{ }^{\mathrm{P}}$ or $\mathrm{R}_{\psi}{ }^{\mathrm{P}} \mathrm{R}_{\zeta}{ }^{\mathrm{P}}$ are the gyradaptive force of an auto-assembling, auto-adaptive, auto-metabolic alternagyrosystem. The trinucleotide is the most unstable and susceptible to change, the dinucleotide $\left(\mathrm{R}_{\xi}{ }^{\mathrm{P}} \mathrm{R}_{\psi}{ }^{\mathrm{P}}\right.$; diquantum) is relativistically more stable, and the mononucleotide $\left(\mathrm{R}_{\xi}{ }^{\mathrm{P}}\right.$; uniquantum) is the most stable. Since the $3^{\text {rd }}$ nucleotide has the broadest genetic information flexibility and the $1^{\text {st }}$ is the most constrained vis-à-vis its encoded amino acid [448], this triquantal organization concomitantly evinces an organizational basis for the triplet codon and intimates its degeneracy and the basis of the wobble [449]. I expand on the specificity of the code in 3.6.

Ribovirogenesis. Whether or not viruses are alive is a matter of much debate and speculation [450]. Furthermore, while there are many ideas related to how viruses evolutionarily originated [451-453], there is no consensus model. Given the emergence of genetic information (ribogyre) within a phospholipid bilayer (phosphogyre) in the absence of cell division (3.8), the ribogyre parsimoniously 
models modern ribovirogenesis — including retroviruses like human immunodeficiency virus [454] —or the origin and evolution of primitive RNA viruses. Notably, many viral RNA assemble as a spherical structure [455], known here as a ribosphere. Finally, the RNA virus life cycle, when viewed in four dimensions, is revealed to be a ribovirogyre.

Nucleotides and Nitrogenous Compounds. The tertiary ribogyre (Figure 2e (iii)) represents the cycling of a gyradaptive triphosphon (3()), modeling the origin, evolution, and existence of a pool of, sets of, or individual nucleosides, nitrogenous compounds, or nitrogenous bases (R) [456,457]. The predicted polymeric signature with the $\mathrm{sub}_{2}$ gyre gyrolink (as per $\mathrm{G}_{\mathrm{XI}}$ ) is identifiable by linearizing the ring structure of adenine:

$$
[R]=\mathrm{N}^{\mathrm{C}} \mathrm{N}^{\mathrm{C}} \mathrm{N}^{\mathrm{C}} \mathrm{N}^{\mathrm{C}} \mathrm{N}^{\mathrm{C}}
$$

where $\mathrm{N}$ is the nitrogen gyromodule and $\mathrm{C}$ is the carbyon gyrolink. The tertiary ribogyre is written in gyrequation form as

$$
\mathrm{R}^{3 \mathrm{P}} \leftrightarrows[\mathrm{R}]+3 \oplus
$$

which, written elementally (excluding trace but physiologically important elements), models

$$
\mathrm{CHNOP} \leftrightarrows \mathrm{CHON}+\mathrm{P}
$$

Molecules that are positioned in the gyrobase of the tertiary ribogyre retain the nitrogen but lack the compositional and thermodynamic signature of the phosphon. These $\mathrm{CHON}$ biomolecules are antioxidants like melatonin [458,459], catecholamines [460], nitrogen heterocycles [461], coenzymes [462], tetrapyrroles [463], xanthines [464], folic acid [465], urate [466], serotonin [467], sphingosine and ceramide [468], and, importantly, amino acids. To these I turn.

Origin and Homochirality of Amino acids. There is no general theory to explain the origin and evolution of amino acids, although the Miller-Urey experiment [469] is frequently cited as a means for their generation. As just alluded, eighteen of the twenty common amino acids have CHON composition, and are thus are modeled as undergoing metabolism in the tertiary ribogyre: electron- and oxyon-mediated catabolism of the carbyon (ribose sugar) and ribon (nitrogenous base; the identifier of the ribogyre as a quantum or particle, Figure 2e (iv)) into linearized, branched molecules. Consistent with their positioning in the tertiary ribogyre, glycine, glutamine, glutamate and aspartate are implicated in the biosynthetic origin of purine and pyrimidine rings [470-472]. Gyrosystem breakdown of guanine at the carbonyl gives

$$
\mathrm{N}^{\mathrm{C}}(\mathrm{N}) \mathrm{N}^{\mathrm{C}} \mathrm{N}^{\mathrm{C}} \mathbf{N}^{\mathrm{CC}}
$$

where $(\mathrm{N})$ is a branched nitrogen bond, and the last four atoms (in bold) are identical to the amino acid backbone with the amino head group, internal carbon, and carboxy terminus. Electron- and oxyonmediated remodeling of uracil and cytosine has potential to generate certain amino acid side groups [473]. The amino acid histidine [474] bears the signature of the nitrogenous base.

The homochirality of amino acids is dispatched by one gyraxiom: IEM that emerges in or is modeled by a tertiary majorgyre spins in the direction of the sub ${ }_{2}$ gyre $\left(\mathrm{G}_{\mathrm{XII}-2}\right)$. In other words, because the carbogyre is a levoragyre, and amino acids are positioned in the tertiary ribogyre, amino acids exist almost exclusively in the L-form. 
Biogeochemical Nitrogen Cycle. Earth's atmosphere is $\sim 80 \% \mathrm{~N}_{2}$. How this came to be is not necessarily clear, although the biogeochemical cycle of nitrogen is indubitably an important aspect of the Earth system [475] and required for the existence of life. Given long-range thermodynamic interaction through the tertiary majorgyre gyrobase $\left(\mathrm{G}_{\mathrm{XI}-1}\right)$, the tertiary ribogyre is the entry point for the biogeochemical nitrogen cycle, with atmospheric nitrogen, $\mathrm{N}_{2}$, and its fixation to $\mathrm{NH}_{4}{ }^{+}$modeled by autocatalysis of the electrogyre, conversion to nitrites and nitrates [476] modeled by the thermodynamic repulsive force of the oxygyre, and assimilation modeled by thermodynamic repulsion by the carbogyre on the nitrogen into amino acids (e.g., aspartic acid, glutamic acid, glycine, alanine, and arginine [477]). Stepping back to the electrogyre and oxygyre, nitrogen cycling can now be modeled as its own set of majorgyres, called nitrogyres:

$$
\begin{gathered}
\text { Primary nitrogyre: } \mathrm{NO}_{3}{ }^{-} \leftrightarrows \mathrm{NO}_{2}^{-}+\mathrm{O}\left(\mathrm{N}^{3 \mathrm{O}} \leftrightarrows \mathrm{N}^{2 \mathrm{O}}+\text { () }\right) \\
\text { Secondary nitrogyre: } \mathrm{NO}_{3}{ }^{-} \leftrightarrows \mathrm{NO}+\mathrm{O}_{2}\left(\mathrm{~N}^{3 \mathrm{O}} \leftrightarrows\left[\mathrm{N}^{\mathrm{O}}\right]+2 \bigcirc\right) \\
\text { Tertiary nitrogyre: } \mathrm{NO}_{3}{ }^{-} \leftrightarrows \mathrm{N}+\mathrm{O}_{3}\left(\mathrm{~N}^{3 \mathrm{O}} \leftrightarrows[\mathrm{N}]_{1-\mathrm{n}}+3 \bigcirc\right)
\end{gathered}
$$

Nitrate $\left(\mathrm{NO}_{3}{ }^{-}\right)$, nitrite $\left(\mathrm{NO}_{2}{ }^{-}\right)$, and nitric acid $(\mathrm{NO})$ are reactive and important inorganic biochemicals $[478,479]$. NO is a biochemical component of L-arginine metabolism [480], additional confirmation that the tertiary ribogyre fits amino acids. In addition to these reactive chemicals, the tertiary nitrogyre gyrobase has three major forms (other allotropes exist as well, all of which are unstable): $\mathrm{N}_{3}$, represents azide, an amine precursor [481] that is highly reactive and unstable (an explosophore); $\mathrm{N}_{1}$ corresponds to elemental nitrogen (modeled by the electrogyre), which by virtue of its trivalence (another triquantal form) rapidly forms $\mathrm{N}_{2}$. The nitrogyre thus represents, models, and explains the $\mathrm{N}_{2}$ and other fundamental nitrogenous compounds that accumulate(s/d) on Earth [482,483].

From RNA to Polypeptides. The ribogyre shows how life uses nucleotides for both information transmission and energy storage. Furthermore, the ribogyre validates the existence of an RNA world prior to the emergence of protein, DNA, and the living cell. Although ribozymes (catalytic RNAs molecules [484]) have been proposed to play an important role in the RNA world [485], this theory shows an alternate view for ribogenesis. One important ribozyme, rRNA [486], catalyzes amide bond formation in protein synthesis and, along with mRNA and tRNA, represents the thermodynamic driving force for the emergence of the next gyrosystem in the evolution of life.

\subsection{Protein}

Despite a great deal of hypothesizing about the origin of the translation complex $[487,488]$, there has not been one idea or model to gain wide scientific approval. What is agreed upon is that polypeptides emerge from within a macromolecular complex of RNAs called the translation apparatus. This nested organization is noteworthy, as it permits the consistent modeling of the aminogyre as emerging from within the ribon.

Before I continue, two points. First, the symbol R refers to, for example, a unique RNA molecule, a pool of the same class of RNAs, an RNA complex, nucleotide, nucleoside, nitrogenous base, and/or the amine group that defines each of these molecules. In other words, the ribon captures a wide range of macromolecules and chemistries, whose identities may be lost to the unified symbolism of 
majorgyres. Second, the aminogyre is a gyromodel that simultaneously fits data related to a polypeptide's evolution on Earth and its present-day functions in the cell.

Specificity of the Genetic Code. Understanding the specificity of the genetic code (introduced in 3.5 ) requires a deconstruction of the primary aminogyre (Figure $2 \mathrm{f}(i)$ ), where

$$
\mathrm{A}^{3 \mathrm{R}} \leftrightarrows \mathrm{A}^{2 \mathrm{R}}+\mathbb{R}
$$

is rewritten as

$$
\begin{gathered}
\mathrm{A}^{\mathrm{R}^{\prime} \mathrm{R}^{\prime \prime} \mathrm{R}^{\prime \prime \prime}} \leftrightarrows \mathrm{A}^{\mathrm{R}^{\prime} \mathrm{R}^{\prime \prime}}+\mathrm{R}^{\prime \prime \prime}, \text { and } \\
\mathrm{R}^{\prime}=\operatorname{mRNA}(\mathrm{s}) \\
\mathrm{R}^{\prime \prime}=\operatorname{tRNA}(\mathrm{s}) \\
\mathrm{R}^{\prime \prime \prime}=\operatorname{rRNA}(\mathrm{s}) \text {, and } \\
\mathrm{A}=\text { amino acid(s) (aa; or amino acid polymer) }
\end{gathered}
$$

Substituting into the gyrequation, I arrive at the following schema:

$$
\text { aa-tRNA/mRNA/rRNA } \leftrightarrows \text { aa-tRNA/mRNA + rRNA }
$$

where aa-tRNA represents charged aminoacyl-tRNAs ([489]; see below). As with other gyrapices, $A^{R^{\prime} R^{\prime \prime} R^{\prime \prime \prime}}$ is unstable. By comparison, the gyrobasal $A^{R^{\prime} R^{\prime \prime}}$ is relativistically more stable, modeling the pool of stably aminoacyl-charged tRNA and mRNA in a ternary complex. (Note that $A^{2 R}$ has potential to model $A^{\mathrm{R}^{\prime} \mathrm{R}^{\prime \prime \prime}}, \mathrm{A}^{\mathrm{R}^{\prime \prime} \mathrm{R}^{\prime \prime \prime}}$, or any other two RNA species.) The gyradaptive ribon $(\mathbb{R})$ depicts rRNA (or any RNA species that impacts the structure of the gyrapical complex). Written another way, ribon repulsion elicits quarternary complex formation,

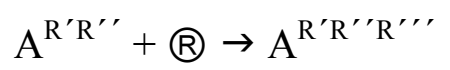

and ribon attraction evicts an adapted ternary complex,

$$
\mathrm{A}^{\mathrm{R}^{\prime} \mathrm{R}^{\prime \prime} \mathrm{R}^{\prime \prime \prime}} \rightarrow \mathrm{A}^{\mathrm{R}^{\prime} \mathrm{R}^{\prime \prime}}+\mathbb{R}
$$

The primary aminogyre thus demonstrates that the aminoacyl-tRNA and mRNA physically co-adapt. The co-gnostic and -mnenomic shaping of these ribonucleotide classes is detectable in codon and anti-codon identity and amino acid specificity (aminognosis and aminomnemesis). Further, the primary aminogyre predicts that this co-adaptational process proceeds vectorially through the rRNA particle - but without amide bond formation. This model is compatible with the co-evolution theory of the genetic code [490,491]. Finally, as there are three defined tRNA occupancy sites (A, P, and $E[492,493])$ in the rRNA, this reveals how amino acids, like the code and the NTP itself, are organized triquantally.

Since gyrequations permit themselves to representing the Matrioshkagyre organization (2.4.1), I can replace the ribons with their specific ribogyre and phosphogyre equations:

$$
\text { mRNA, tRNA, and rRNA }=\mathrm{R}^{3 \mathrm{P}} \leftrightarrows\left[\mathrm{R}^{\mathrm{P}}\right]+2 \odot
$$

18/20 amino acids $(\mathrm{A} ;$ see below $)=\mathrm{R}^{3 \mathrm{P}} \leftrightarrows[\mathrm{R}]+3 \circledast$ 
Substituting appropriately, the primary aminogyre could be thought of as:

$$
\left(\mathrm{R}^{3 \mathrm{P}} \leftrightarrows[\mathrm{R}]+3 \odot\right)^{3\left(\mathrm{R}^{3 \mathrm{P}} \leftrightarrows\left[\mathrm{R}^{\mathrm{P}}\right]+2 \odot\right)} \leftrightarrows\left(\mathrm{R}^{3 \mathrm{P}} \leftrightarrows[\mathrm{R}]+3 \odot\right)^{2\left(\mathrm{R}^{3 \mathrm{P}} \leftrightarrows\left[\mathrm{R}^{\mathrm{P}}\right]+2 \odot\right)}+\left(\mathrm{R}^{3 \mathrm{P}} \leftrightarrows\left[\mathrm{R}^{\mathrm{P}}\right]+(\mathrm{P})\right.
$$

but even this representation excludes information. Nevertheless, with this provisional picture, I show that changes in orthophosphate levels impart changes in nucleotide salvage pathways [494] which, in turn, impart changes on RNA levels and composition and the metabolism of amino acids, which ultimately translates to the specificity of the genetic code.

Sulfated Amino Acids and Biogeochemical Sulfur Cycle. The vast majority of the sulfur in the living cell is found within polypeptides as cysteine and methionine [495]. Rounding out the positioning of biogeochemical cycles [496], I model the aminogyre as the input point for the sulfur cycle [497,498]: mineralization of organosulfur compounds and metabolism of elemental sulfur to $\mathrm{H}_{2} \mathrm{~S}$, hydrogen sulfide [499], is modeled as a consequence of the repulsive electrogyre; oxidation to $\mathrm{HSO}_{4}$, sulfate [500], is modeled by the repulsive oxygyre; sulfur assimilation to organic and nitrogenous sulfhydryl [501] with sulfur being metabolized into the amino acids cysteine (Cys), homocysteine, methionine (Met), and taurine [502,503] - is modeled by the creatodestructive, expansocontractive, and attractorepulsive forces of the carbogyre through the tertiary ribogyre and into the aminogyre. The cycling of the full complement of amino acids, peptides, and proteins (proteome) within, between, and among cells in the biosphere is called the macroaminogyre.

Positioning Met and Cys in the aminogyre abides by $\mathrm{G}_{\mathrm{IX}}-\mathrm{a}$ novel IEM must emerge in a focagyre-since macromolecules containing the element sulfur emerge in the evolution of life. Notably, Met in particular initiates the polypeptide [504], indicating the evolutionary necessity for novel IEM. Finally, given that the ribogyre is a levoragyre, it exerts a left-handed chemosynthetic force $\left(\mathrm{G}_{\mathrm{XII}}\right)$ on Met and Cys; thus these amino acids are exclusively L-form in living systems. This theory eliminates the problem of homochirality of all amino acids, given aminogyre emergence from the phosphogyre and ribogyre; moreover, it is compatible with ideas of phosphate- and RNA-dependent mechanisms for generating amino acid chirality [505,506].

Protein synthesis and degradation. An accurate theory of life must have an explanation of how polypeptides are created and how they are destroyed. The current models to explain these phenomena are largely unrelated: at its core, protein synthesis involves a quarternary complex of mRNA, aa-tRNA, and rRNA [507] and protein turnover involves either the autophagic-lysosomal pathway [508] or targeting by specific protein enzymes or multi-protein complexes called proteases (e.g., the proteasome [509]). I thus applied the aminogyre framework to unify these processes. The secondary aminogyre (Figure $2 \mathrm{f}(\mathrm{ii})$ ) is written as

$$
\mathrm{A}^{3 \mathrm{R}} \leftrightarrows\left[\mathrm{A}^{\mathrm{R}}\right]+2 \mathbb{R}
$$

which models:

$$
\text { aa-tRNA/mRNA/rRNA } \leftrightarrows \text { aa-tRNA }+ \text { mRNA/rRNA }
$$

As shown above, the simplest unit is aminoacyl-tRNA. However, as $\mathrm{G}_{\mathrm{X}}$ dictates-i.e., in a gyronexus, the gyrolink $\mathrm{R}$ is the dIEM of the subgyre - $\mathrm{R}$ corresponds to the amide bond $(\mathrm{N})$ that links amino acids: 


$$
\left[A^{R}\right]=A^{R} A^{R}, A^{R} A^{R} A^{R}, A^{R} A^{R} A^{R} A^{R} A^{R} A^{R} \cdots
$$

which is

$$
\left[a^{\mathrm{N}}\right]=\mathrm{aa}^{\mathrm{N}} \mathrm{aa}^{\mathrm{N}}, \mathrm{aa}^{\mathrm{N}} \mathrm{aa}^{\mathrm{N}} \mathrm{aa} a^{\mathrm{N}}, \mathrm{aa}^{\mathrm{N}} \mathrm{aa}^{\mathrm{N}} \mathrm{aa}^{\mathrm{N}} \mathrm{aa}^{\mathrm{N}} \mathrm{aa}^{\mathrm{N}} \mathrm{aa} a^{\mathrm{N}} \ldots
$$

Based upon this model, the gyrolink imports the information and energy from the tRNA (see 3.5); alternatively, nitrogenous bases impute genetic information into the amide bond. Making the gyrequation unidirectional, protein synthesis is modeled as diribon attraction,

$$
\mathrm{A}^{3 \mathrm{R}} \rightarrow\left[\mathrm{A}^{\mathrm{R}}\right]+2 \mathbb{R}
$$

wholly consistent with release of the nascent polypeptide - called here an aminonexus-from the rRNA and mRNA, with tRNAs displaced upon amide bond formation. Protein turnover, in contrast, is modeled as gyradaptive repulsion by the diribon,

$$
\left[\mathrm{A}^{\mathrm{R}}\right]+2 \mathbb{R} \rightarrow \mathrm{A}^{3 \mathrm{R}}
$$

In other words, this theory of life predicts that RNA, nucleotides, organic bases have prominent and direct roles in protein metabolism. This protease-independent model of protein structure remodeling is consistent with data suggesting that changing levels of water (oxygyre), hormones and fatty acids (carbogyre), phosphorylation (phosphogyre), and amino acids (ribogyre) directly impact protein stability [509-513]. This model also provides a unique perspective on polypeptide evolution: ribon (nucleotides, RNA, amine)-based cycling through and into the aminonexus (aminocycle) allows the generation and feedback of domains and enzymatic activities into subgyres. In other words, theory explains the origin, evolution, and structure of novel protein motifs, domains, and folds [514,515]. Moreover, the proximity of the aminonexus to the diribon singularity determines its rates of turnover and evolutionary change [516]. Combining and compressing the primary and secondary aminogyres shows that

$$
\mathrm{A}^{2 \mathrm{R}} \leftrightarrows\left[\mathrm{A}^{\mathrm{R}}\right]+\mathbb{R}
$$

an alternagyre that permits modeling of aminognosis of any two ribons (2R) - mRNA and tRNA, mRNA and rRNA, tRNA and rRNA, or any other RNA classes, species, or elements - with a polypeptide or amino acid (A). This $1 \% 2^{\circ}$ alternagyre, along with the primary and secondary aminogyre (and subsumed gyres), provides a framework for understanding the origin of the translation apparatus in the evolution of life and additional modes of specificity of the genetic code.

Aminoacyl-tRNA Metabolism. To this point, I have not explained the origin and emergence of aminoacyl-tRNAs. Most current ideas related to their existence invoke aminoacyl-tRNA synthetases, proteins that attach an amino acid to a cognate tRNA [517,518]. Since this class of enzymes cannot emerge without translation, there is a chicken-and-egg enigma. Addressing this puzzle, by equating the secondary and tertiary aminogyres (Figure $2 \mathrm{f}($ iii)), I have,

$$
\left[\mathrm{A}^{\mathrm{R}}\right]+2 \mathbb{B} \leftrightarrows \mathrm{A}^{3 \mathrm{R}} \leftrightarrows[\mathrm{A}]+3 \mathbb{R}
$$

which, following compressing and balancing, is the $2 \% / 3^{\circ}$ alternagyre,

$$
\left[\mathrm{A}^{\mathrm{R}}\right] \leftrightarrows[\mathrm{A}]+\mathbb{B}
$$


Based upon sub ${ }_{2}$ gyre tertiary majorgyre gyrolink $\left(\mathrm{G}_{\mathrm{XI}}\right)$, the gyrobasal $[\mathrm{A}]_{1}$ is, for example, one or a pool of individual aa with a phosphon (aa P), which represents

$$
\text { aa-tRNA } \leftrightarrows \text { aa } \sim \mathrm{P}+\text { tRNA }
$$

Thus, theory shows that in the evolutionary absence of the aminoacyl-tRNA synthetases, the ribon is the attractorepulsive force responsible for both creation and destruction of aminoacyl-tRNAs.

Higher-order Protein Structure. Similar to other gyronexuses, polypeptides assemble into $\alpha$-helices ([519]; Figure 4f), $3{ }_{10}$-helices [520], П-helices [521], $\beta$-sheet helices [522]-largely D-form to homeostatically balance the L-amino acids $\left(\mathrm{G}_{\mathrm{XII}-3}\right.$; Figure $\left.4 \mathrm{f}\right)$. These structures are theoretically unified in the term aminohelices. Aminohelices assemble into coils, fibers, and aggregates [523] that are termed aminomatrices; these exist both intracellularly (e.g., higher-order micofilaments [524], microtubules [525], intermediate filaments [526]) and extracellularly (e.g., collagen [527], fibronectin [528,529], and laminin [530]). Finally, polypeptides assemble into aminomatrices that are architecturally spherical or ovoid such as a viral capsid [531] and clathrin cage [532]; these structures are aminospheres.

Protein Folding. Anfinsen's classic experiment - in which a denatured ribonuclease refolded properly, restoring catalytic activity [533] — led to many questions about how an unfolded polypeptide "remembers" its structure. Anfinsen himself suggested that the primary amino acid sequence determines native structure [534], but this idea doesn't explain how a primary sequence initially acquires its folded state. This so-called protein folding problem [535] is resolved by this theory. Given the gnostic and mnemonic properties of the gyromodel (2.4.4), each of these gyrosystems [536] learns a particular spatiotemporal orientation, contextualization, and function, and, once stored and templated, remembers and restores it under appropriate thermodynamic conditions.

Nucleoproteins and Post-translational Modifications. The secondary aminogyre accounts for the origin and emergence of three classes of aminonexuses vital to nucleotide biochemistry: (i) nucleotide sensor enzymes (e.g., ribonucleotide reductases [537]); (ii) nucleotide-modifying enzymes (e.g., protein kinases and phosphatases [538-540], and DNA and RNA polymerases [541-544], nucleases [545-547], helicases [548]); and (iii) nucleotide-binding proteins (sequence-specific (e.g., transcription factors [549]) and sequence-nonspecific (e.g., histones [550]). I return to these three classes in 3.7. Polypeptides undergo different types of modifications [551-556]; the theoretical framework fits these as well [557].

Phosphoproteins, Ribonucleoproteins, and Membrane Proteins. Recall that a particle has quantum potential $\left(\mathrm{G}_{\mathrm{III}}\right)$, meaning that $\mathrm{R}$ has the potential to represent many distinct molecules, as does A. Because the phosphon is the gyrolink of the tertiary aminogyre $\left(\mathrm{G}_{\mathrm{XI}}\right)$, the $2^{\circ} / 3^{\circ}$ alternagyre accounts for three distinct properties and characteristics of polypeptides. First, in the gyrapex, because phosphons in nucleotides (NTP, NDP, NMP) interact with aminons ((Figure $2 \mathrm{f}(i v)$; quantized particle that is the aminogyre $\left(\mathrm{G}_{\mathrm{I}}\right)$ ), this models the establishment, maintenance, and chemosensory qualities of a nucleotide binding motif [558]. Second, aminonexus binding to ribonexuses is modeled here as well: $\left[\mathrm{A}^{\mathrm{R}}\right]_{\mathrm{n}}$ represents these RNA-protein complexes called ribonucleoproteins, where $[\mathrm{A}]$ is one or more polypeptides that a gyrolinked by phosphates (e.g., post-translational modifications, sugar $\sim \mathrm{P}$, or polyP) gyrobasally: 
This schema fits numerous ribonucleoprotein complexes [559-567]. Phosphoproteins can also be viewed from the standpoint of the triribon singularity (Figure $3 \mathrm{~g}$ ). Third, the tertiary majorgyre facilitates a new understanding of polypeptide-phosphomembrane organization. Given that

$$
\begin{gathered}
{[\mathrm{A}]=\mathrm{A}^{\mathrm{P}}, \mathrm{A}^{\mathrm{P}} \mathrm{A}^{\mathrm{P}}, \mathrm{A}^{\mathrm{P}} \mathrm{A}^{\mathrm{P}} \mathrm{A}^{\mathrm{P}} \mathrm{A}^{\mathrm{P}} \ldots, \text { and }} \\
\mathrm{P}=\text { phospholipids and phosphonexuses }
\end{gathered}
$$

this models how a polypeptide (quantized in A), establishes direct relationships with a phosphomembrane, interdigitating with the surface phosphates. This clarifies the membrane protein folding problem [568]. Many hydrophobic and membrane-anchored macromolecules (e.g., channels [569], pores [570], basal body [571]) are positioned here [572].

Non-ribosomal Peptides and Sulfated Compounds. I can now fit nonribosomal peptides (NRPs, e.g., antibiotics, siderophores, cytostatics; [573]) — secondary metabolites produced by a variety of microorganisms, many of which participate in intra- and intercellular signaling [574]. The translation apparatus does not generate NRPs but rather, it is thought, enzymes do [575]. Here, I model NRPs origins as

$$
\mathrm{NRP} \leftrightarrows \mathrm{aa}^{\mathrm{x}}+\mathbb{R}
$$

which fits onto $\left[\mathrm{A}^{\mathrm{R}}\right] \leftrightarrows[\mathrm{A}]+\mathbb{B}$, where $\mathbb{R}$ is the gyradaptive force (e.g., NTPs), aa is any amino acid [576,577], and ' $x$ ' - given long-range thermodynamic interactions of greater exergy $\left(\mathrm{G}_{\mathrm{XI}-1}\right.$ and $\mathrm{G}_{\mathrm{XIII}}$ - - denotes any chemical modification or solution $\left(\mathrm{H}_{2} \mathrm{O}\right)$ with sufficient potential energy to facilitate NRP metabolism. The permutability of the gyrosystem, along with its adaptive capacity, reveals how antibiotics, over time, lose their efficacy and specificity [578]. [A] also positions phosphorus- and nitrogen-free molecules (C, H, O, and S) like sulfolipids [579] due to carbogyre attractorepulsion on the tertiary aminogyre. Additional evidence validating this thermodynamic carbyon-aminon relationship is found in aminocarbomatrices called peptidoglycans [580].

CHNOPS. How and why life is predominantly made up of hydrogen, oxygen, carbon, phosphorus, nitrogen, and sulfur is an unanswered question [581]. I show that the electrogyre $(\mathrm{H}$, and all other elements), oxygyre $(\mathrm{O})$, carbogyre $(\mathrm{C})$, phosphogyre $(\mathrm{P})$, ribogyre $(\mathrm{N})$ and aminogyre $(\mathrm{S})$ provide a coherent theoretical answer for how and why the living cell is $98 \%$ CHNOPS.

From Protein to DNA. The amino acid is the molecular building block for the polypeptides that exist in all life forms known to science. With the ribogyre and aminogyre, I have provided an axiomatically constrained and empirically consistent system for understanding the origin and evolution of these biomolecules. The aminogyre makes some very profound and testable predictions about the specificity of the genetic code and how proteins behave, lengthen and shorten, and fold and unfold in response to physical and biometabolic changes or changes in genetic information content of RNA.

When considering the next evolutionary bound towards the origin of life, my attention turned to one particular class of proteins. The enzyme RNR is a crucial protein in the evolution of life because it and it alone performs an essential biochemical reaction: RNR converts a ribonucleotide to a deoxyribonucleotide [582]. Without this reaction, DNA would not exist and the living cell as I know it would not emerge. Thus, the RNR protein family, along with a cadre of nucleoproteins, is part and 
parcel of the very existence of genes and genomes - a veritable molecular bridge between the RNA and DNA worlds [583-585].

\subsection{DNA}

DNA is arguably the molecular capstone in the evolution of life. In revealing the structure of DNA, the "secret of life [586]," Crick and Watson set the stage for a new generation of scientists to find that there was a seemingly endless quest towards unraveling a profound mystery enshrouding that secret [587]. In this subsection, I fit some of the most important facts related to DNA onto a gyrosystem called the genogyre. The genogyre provides novel viewpoints on the origin and evolution of genes, genomes, and chromosomes. Because the aminogyre is nested within the genogyre, the Matrioshkagyre organization is a spatiotemporal heuristic for how proteins regulate DNA structure and function.

Deoxynucleotides and DNA Cis-Acting Elements. The primary genogyre (Figure $2 \mathrm{~g}(i)$ ), fits the evidence about deoxynucleotide origin and evolution, where

$$
\mathrm{D}^{3 \mathrm{~A}} \leftrightarrows \mathrm{D}^{2 \mathrm{~A}}+\text { (A) }
$$

$$
\begin{gathered}
\mathrm{D}=\text { deoxynucleotide triphosphates (dNTPs: dATP, dGTP, dCTP, dTTP) } \\
\text { diphosphates (dNDPs), and monophosphates (dNMPs) } \\
\text { double-stranded (ds) and single-stranded (ss) DNA }
\end{gathered}
$$

$\mathrm{A}=\mathrm{A}^{\prime}$, nucleic acid sensors; $\mathrm{A}^{\prime \prime}$, modifiers; and A"', interactors (3.6); any other germane protein.

This quarternary complex of DNA/sensor/modifier/interactor $\left(\mathrm{D}^{3 \mathrm{~A}}\right.$; gyrapex $)$ is predicted to be unstable, with any one of the three nucleoproteins disassembling and cycling through the gyrosystem. In this regard, this model fits the known evidence about RNR(s) as sensing and converting

$$
\begin{aligned}
& \mathrm{NMP} \rightarrow \mathrm{dNMP} \text { and } \\
& \mathrm{NDP} \rightarrow \mathrm{dNDP}[582]
\end{aligned}
$$

nucleotide kinase(s) to convert

$$
\begin{gathered}
\mathrm{dNMP} \rightarrow \mathrm{dNDP} \text { [588] and } \\
\mathrm{dNDP} \rightarrow \mathrm{dNTP} \text { [589] }
\end{gathered}
$$

and nucleotide-binding protein(s) to store, remodel, and regulate (3.6) dNTPs and DNA. Vectorially, modeling the interactor $\left(\mathrm{A}^{\prime \prime \prime}\right)$ cycling,

$$
\mathrm{D}^{\mathrm{A}^{\prime} \mathrm{A}^{\prime \prime}}+\mathrm{A}^{\prime \prime \prime} \rightarrow \mathrm{D}^{\mathrm{A}^{\prime} \mathrm{A}^{\prime \prime} \mathrm{A}^{\prime \prime \prime}}
$$

depicts genognosis, the process by which the deoxynucleotide or DNA receives and interprets IEM from the gyradaptive, repulsive aminon, and

$$
\mathrm{D}^{\mathrm{A}^{\prime} \mathrm{A}^{\prime \prime} \mathrm{A}^{\prime \prime \prime}} \rightarrow \mathrm{D}^{\mathrm{A}^{\prime} \mathrm{A}^{\prime \prime}}+\mathrm{A}^{\prime \prime \prime}
$$

depicts genomnemesis, where the deoxynucleotide or DNA retains information. Cycling of any aminon models its co-adaptational relationships with a ternary complex. Given that D accounts for ssDNA and dsDNA and given the quantal depth of the aminon, $\mathrm{A}^{\prime \prime}$ fits an extremely large yet bounded 
number of trans-acting DNA-binding proteins [590,591]. Thus, this simple model for protein-DNA learning and memory explains how a protein physically identifies and targets (learns and remembers) a very specific cis-acting sequence (e.g., promoters, enhancers, terminators [592]). Continuous macrocosmic genognosis and genomnemesis is consistent with bioinformatic evidence [593-596] showing that cis-acting elements and motifs change in the evolutionary tree branches of life.

Chromosomes and Chromatin. The packaging of DNA by proteins occurs in all kingdoms of life. The secondary genogyre (Figure $2 \mathrm{~g}(\mathrm{ii})$ ) can be written as

$$
\mathrm{D}^{3 \mathrm{~A}} \leftrightarrows\left[\mathrm{D}^{\mathrm{A}}\right]+2 \oplus
$$

Here, the gyrobasal $\left[\mathrm{D}^{\mathrm{A}}\right]$ represents what is theoretically called a genonexus. A genonexus is a length of DNA (gyromodule) gyrolinked $\left(\mathrm{G}_{\mathrm{X}}\right)$ by proteins (aminons that are aminonexuses). In this scenario, the gyrolink is $\mathrm{A}^{\prime \prime \prime}$, which models nucleoid proteins [597] in eubacteria and chromatin proteins (especially histones [598]) in archaebacteria and eukaryotes. In eukaryotes in particular, $\mathrm{D}^{\mathrm{A}}$ is a mononucleosome, 146 base pairs of DNA spiralling around an octamer of histones $\mathrm{H} 2 \mathrm{~A}, \mathrm{H} 2 \mathrm{~B}, \mathrm{H} 3$, and H4; (Figure 4g; [218]), $\mathrm{D}^{A} \mathrm{D}^{\mathrm{A}}$ is a dinucleosome [599], and $\mathrm{D}^{\mathrm{A}} \mathrm{D}^{\mathrm{A}} \mathrm{D}^{\mathrm{A}} \mathrm{D}^{\mathrm{A}} \mathrm{D}^{\mathrm{A}} \ldots$; ; any longer genonexus models "beads on a string [600]."

Although I modeled the diaminon that is the thermodynamic driving force for genonexus formation as $\mathbf{A}^{\prime}$ and $\mathbf{A}^{\prime \prime}$ (2ब $\left.=\mathbf{A}^{\prime} \mathbf{A}^{\prime \prime}\right)$, the secondary genogyre fits any gyradaptive diaminon. For this schema, genonexus packaging and organization is modeled vectorially:

$$
\mathrm{D}^{\mathrm{A}^{\prime} \mathrm{A}^{\prime \prime} \mathrm{A}^{\prime \prime \prime}} \rightarrow\left[\mathrm{D}^{\mathrm{A}^{\prime \prime \prime}}\right]+2 \text { (A) }
$$

and breakage, remodeling, and mobilization, is depicted as:

$$
\left[\mathrm{D}^{\mathrm{A}^{\prime \prime \prime}}\right]+2(A) \rightarrow \mathrm{D}^{\mathrm{A}^{\prime} \mathrm{A}^{\prime \prime} \mathrm{A}^{\prime \prime \prime}}
$$

Elaborating from above, $\mathbf{A}^{\prime \prime}$, models trans-acting factors, specifically activators [601,602], repressors [603], chromatin remodeling factors [604], among others. $\mathbf{A}^{\prime \prime}$ represents the full complement of DNA-modifying enzymes (3.6), all of which are demonstrably participants in the generation and organization of the genonexus. Indeed, DNA repair [605], recombination [606], transposition [607,608], and sequence rearrangements [609] are all modeled onto the secondary genogyre as the gyradaptive effects of the diaminon singularity. Shorthand, this would be:

$$
\text { chromatin } \leftrightarrows \text { nucleosomal DNA + protein sensors/modifiers }
$$

DNA Structure. DNA is a double helix of antiparallel dNMP single strand polymers (a chemically modified ribonexus called a deoxyribonexus; not to be confused with the genonexus, which is the deoxyribonexus dynamically gyrating around aminonexuses). Note that the molecular genetical homeostasis of the antiparallel deoxyribonexuses orbiting the aminon singularity is comparable to the biophysical homeostasis of the antiparallel phosphonexuses orbiting the carbyon singularity (3.4). Although left-handed A-form DNA can be engineered, only right-handed B- and Z-form DNA occur naturally [610,611]. DNA is exclusively right-handed helix in life-because the aminogyre is righthanded $\left(\mathrm{G}_{\mathrm{XII}}\right)$. Moreover, DNA wraps around the histone octamer in only a left-handed fashion due to oscillating chiralities during gyrosystem complexification $\left(\mathrm{G}_{\mathrm{XII}-3}\right.$; Figure $\left.4 \mathrm{~g}\right)$. Keeping with the theoretical vernacular, then, the genonexus is visibly a genohelix [612]. As the genohelix gyrates upon 
itself, it forms more and more complex genomatrices, the higher order structures known as the $30 \mathrm{~nm}$ solenoid [613], chromatin loops and fibers [614], and mitotic chromosomes [615].

DNA Virus. Given that RNA is the evolutionary predecessor of DNA, a common theme in evolutionary virology is that DNA viruses are evolved from RNA viruses [616]. Being that the deoxyviral particle is inert without the living, dividing cell, the second genogyre models the generation of the viral genonexus:

Viral genome/3A $\leftrightarrows$ Viral genome/packaging proteins $+2 \mathrm{~A}$

This simple model thus portrays the origin and evolution of all DNA viruses; the logical neologism for such a quantized DNA-protein (or RNA-protein) complex in the gyrobase is a "viron". In this regard, the large-scale cycling and metabolism of virons through genomes and cycling of genomes throughout the biosphere is modeled by a macrogenogyre. The genogyre and ribogyre demonstrate that DNA and RNA viruses, respectively, emerge prior to the dividing cell and are a natural consequence of universal expansion towards the origin of life.

Origin of DNA Content, Mutations, and Other Problems. How new open reading frames-regions of the genome that are complementary to RNA sequences-come into existence is a core problem of evolutionary biology and the subject of intense phylogenetic and bioinformatic study [617,618]. One favored explanation is Ohno's gene duplication model [619,620]. Still, in the spirit of scientific transparency and honesty, the duplication idea does not address how the first or novel genes emerge. To address this dilemma, I applied the genogyre accordingly. In the tertiary genogyre (Figure $2 \mathrm{~g}$ (iii)), the gyrobasal [D] loses support of the triaminon, and takes on direct thermodynamic relationships with the ribon $\left(\mathrm{G}_{\mathrm{XI}}\right)$. This ribon-deoxyon interaction models how ribons or ribonexuses exert the attractorepulsive force on the genons (the quantal/particulate model of the genogyre; Figure $2 \mathrm{~g}(i v)$ ), literally "linking" them together:

$$
[D]=D_{R}, D_{R} D_{R}, D_{R} D_{R} D_{R}, D_{R} D_{R} D_{R} D_{R} \cdots
$$

where R = individual NTPs, exons, introns, and regulatory RNAs. In turn, D models the corresponding dNTPs and DNA sequences. This theoretical relationship thus flips the conventional view on its head: RNA is the unstable, ever-changing template upon which the gene and genome forms, expands, and adapts. Compressing the secondary and tertiary genogyres, I have

$$
\left[\mathrm{D}^{\mathrm{A}^{\prime \prime \prime}}\right] \rightarrow[\mathrm{D}]+\text { (A) }
$$

a $2^{\circ} / 3^{\circ}$ alternagyre that shows how the aminon singularity attracts and unfolds the gyrapical genonexus (DNA-protein), thereby permitting interface with the RNA template. The opposing directionality

$$
[\mathrm{D}]+\mathbb{A} \rightarrow\left[\mathrm{D}^{\mathrm{A}^{\prime \prime \prime}}\right]
$$

shows how the aminon repels the gyrobasal genonexus (DNA-RNA) back to its high energy state, evicting the RNA. A full cycle, a genocycle, permits the adaptation of genic and genomic (coding and non-coding) sequences. In other words, RNA-directed changes to the DNA sequence undergo proteinaceous genognosis (where $\left[\mathrm{D}^{\mathrm{A}^{\prime \prime}}\right]$ is the gyrapex, the learning gyrostate of this alternagyre) and ultimately are genomnemonically stored (where $\left[\mathrm{D}^{\mathrm{A}^{\prime \prime \prime}}\right]$ is the gyrobase, the memory gyrostate of the secondary genogyre). The theoretically-defined mnemonic character of DNA-protein is more stable 
than that of DNA-RNA, as the exergy and attractorepulsive effects of the aminogyre are relativistically less those of than the ribogyre (see $\mathrm{G}_{\mathrm{XIII}}$ ).

This model (also see Figure $3 \mathrm{~h}$ ) has the potential to facilitate understanding of a variety of molecular genetic problems. For example, it resolves the origin and evolution of genes and gene families [621,622], origin of intronic sequence in RNA and DNA [623,624], directed mutation controversy [625-627], why 80-90\% of a genome has transcriptional output [628], transcription-associated recombination [629], how RNA mediates epigenetic reprogramming of DNA [630], RNA-templated DNA repair [631], site-specific changes in viral genomes [632,633], and, since RNA harbors the genetic information memory for templating DNA, how lateral gene transfer is widespread between, among, and within different genomes and differing organisms [634-636].

dNTP Pools. Regulating the levels of dNTP pools is fundamental for proper cell function [637]. Moreover, DNA replication - which is necessary for fidelitous cell division - is preceeded by a wave of dNTP accumulation [638] that is tightly regulated [638-640]. In the tertiary genogyre, [D] also models the thermodynamic relationship between the stoichiometric levels and pools of NTPs (ribogyre) and dNTPs (genogyre) unincorporated in RNA and DNA, respectively. This theoretically defined dNTP-NTP feedback jives with the empirical necessity of the chemical energy from ribonucleotides to drive biosynthesis and transport of sugars, membranes, and organelles (3.6) that concresce as new cell material. In the $2 \% 3^{\circ}$ alternagyre, the A that cycles corresponds to RNRssensing levels of dNTPs and NTPs - and other protein sensors such as components of the DNA damage machinery that work during cell cycle checkpoints [641,642].

Flow of Molecular Genetic Information. The current idea for how genetic information flows in cells involves only three components: DNA, RNA, and protein. While this reductionist idea has been powerful, there have been calls of a conceptual crisis $[643,644]$ and for shifts to systems thinking [645]. In other words, these critics imply that understanding the flow of genetic information requires understanding more than just genetic information. As modeled by this interdigitated theoretical framework (Figure 5), genetic information flows coherently from biochemical and biophysical IEM:

$$
\begin{aligned}
\rightarrow \text { electron } \rightarrow \text { water } & \rightarrow \text { organic matter } \rightarrow \text { phosphochemistry and membranes } \\
& \rightarrow \text { RNA } \rightarrow \text { protein } \rightarrow \text { DNA } \rightarrow \text { cell }
\end{aligned}
$$

The relationships of gyrosystems - as shown in the flow diagram - solves many of the unsolved questions in molecular biology [646]. Moreover, the flow diagram provides an alternative perspective to the central dogma, Mendelian genetics [647,648], neo-Darwinian selection of random mutations [649], and selfish gene theory [650] on matters such as genomic stability [651,652], adaptability [653], and inheritance [654].

From DNA to the Living Cell. I have fit the modern evidence related DNA to the genogyre and have theoretically confirmed the nature and composition of the DNA world that existed in evolution of life on Earth [584]. With the genogyre, I have a system of unreplicated DNA within a phosphomembranous sac; in other words, the genogyre does not explain life as I know it. Because a correct theory of life must explain not just how but why a living cell divides, I now turn to DNA replication and cell division. 
Figure 5. Left-to-right theoretical framework. The arrowheads between the gyrosystems (center flow line) represent both the evolutionary process leading up to the origin and evolution of cells and how existing cells work. The self-directed arrows that are above and below the gyrosystems represent autoregulation. The arrowed lines above the center line depict the feedforward between and among gyrosystems; those below the line depict feedback. The gyrosystem interactions discussed the most in the text are labeled as dark lines. The dotted lines represent empirically definable or predicted gyrosystem flow. Those arrowheads that flow into the electrogyre (the photon from the left) and flow out of the cellulogyre (to the right) depict the evolutionary steps prior to and following the origin of visible matter and the cell, respectively; these are either briefly mentioned or not discussed in this study. Please note the unity of reality and life as revealed by this theory.

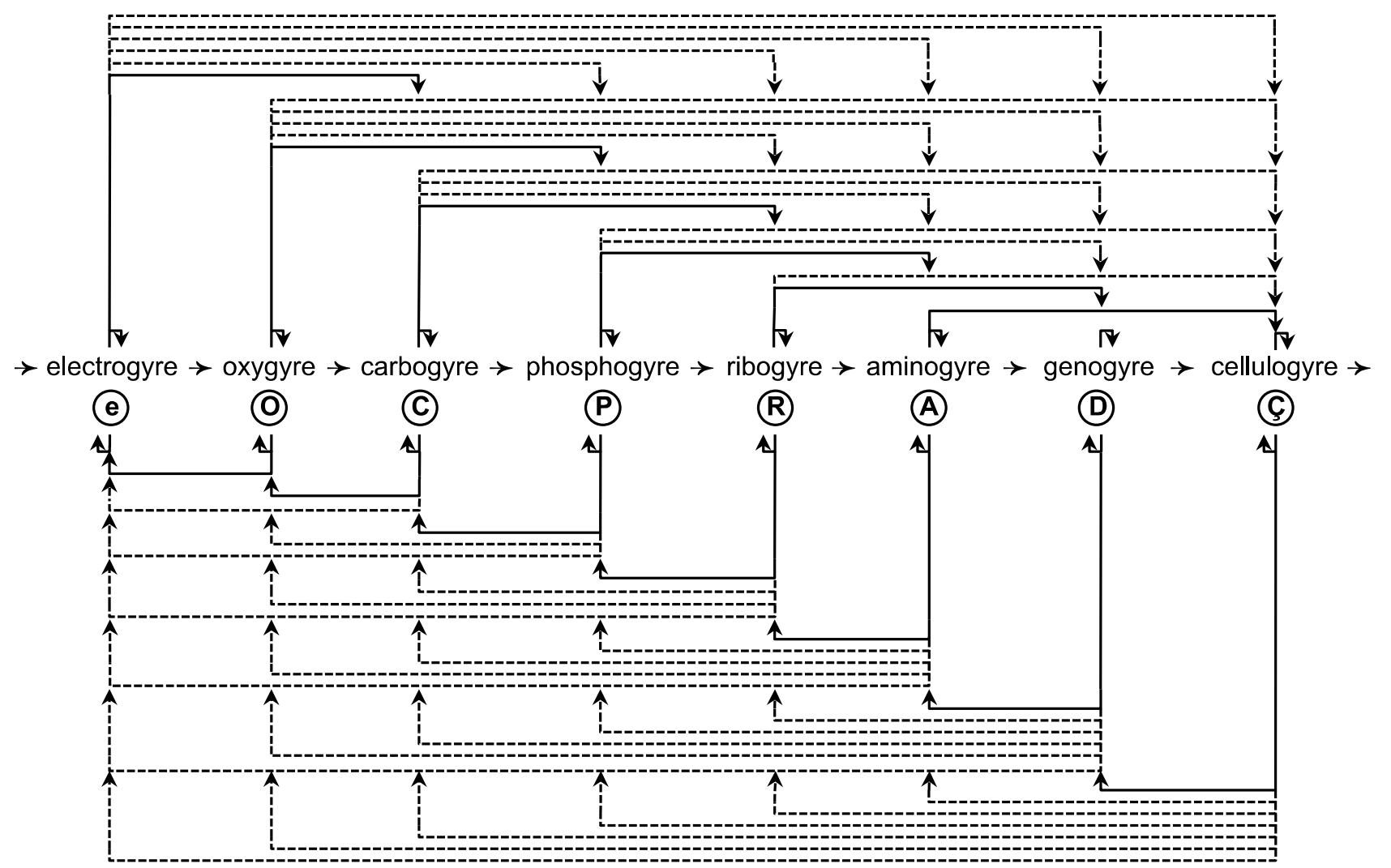

\subsection{Cell}

A vast expanse of the scientific firmament implicitly accepts two core premises of cell theory [655]: (i) the cell is the basic unit of life; and (ii) all cells emerge from other cells by division. While the first premise of cell theory is definitionally confining, it is not theoretically problematical. The second premise, however, is deeply troubling, because it implies that the origin of first cell is impossible to explain by reductio ad absurdum. In considering the origin of life, I have already accounted for and modeled empirical evidence related to the physical, chemical, biochemical, and molecular systems upon which a living cell relies (3.1-3.7). While life can and does exist in a quiescent state for an extended period of time as a spore [656] or in a terminally differentiated state, these cellular forms emerge only from a cell that has potential to divide. Thus, in order to model the origin of life, I had to model the origin of the first cell division. The cellulogyre (Figure $2 \mathrm{~h}$ ) is a gyrosystem that reveals that 
the first cell division on Earth was executed in a fashion identical to a dividing cell that an investigator examines in this very epoch of basic and clinical research.

DNA Replication and Cell Division. During the genesis, repair, and remodeling of genome sequence and order, the pool of unincorporated dNTPs expands within the gyrobase of the tertiary genogyre (3.7). However, as the other gyrosystems that are subsumed by the genogyre are likewise expanding, and the genogyre is the end point of IEM deposition, the genogyre shunts IEM back within itself - one of the most prominent examples of this is phosphogyre expansion, as new membrane synthesis and expansion occurs during the process leading up to cell division $[657,658]$. In both the hapcellulogyre (Figure $2 \mathrm{~h}(\mathrm{i}) ; 1 \mathrm{~N}$, one set of homologous chromosomes (haploid)) and dipcellulogyre (Figure 2h (ii); 2N, two sets of chromosomes (diploid)), respectively, DNA replication $[659,660]$ is modeled as repulsion by the (di)genon, the thermodynamic singularity within the cell (Figures $2 \mathrm{~g}(\mathrm{iv}$ ) and $2 \mathrm{~h})$ :

$$
\begin{gathered}
{\left[C^{\mathrm{D}}\right]+\left(D \rightarrow C^{2 \mathrm{D}}\right.} \\
{\left[\mathrm{C}^{2 \mathrm{D}}\right]+2 \mathbb{D} \rightarrow \mathrm{C}^{4 \mathrm{D}}}
\end{gathered}
$$

and cell division and nucleoid or chromosome segregation [661,662], by its attractive force:

$$
\begin{gathered}
C^{2 \mathrm{D}} \rightarrow\left[C^{\mathrm{D}}\right]+(\mathrm{D}) \\
\mathrm{C}^{4 \mathrm{D}} \rightarrow\left[\mathrm{C}^{2 \mathrm{D}}\right]+2 \mathbb{D}
\end{gathered}
$$

Quite parsimoniously, the hapcellulogyre models the replicated DNA state $\left(C^{2 \mathrm{D}}\right.$; gyrapex) and the pre-replicated state $\left(C^{\mathrm{D}}\right.$; gyrapex) in archaebacteria [663], eubacteria [664,665], plastids and mitochondria [666,667], and haploid eukaryotes. The gyradaptor, (D, represents the $\mathrm{G}_{0}$ state [668]the non-replicative, quiescent, membrane-encapsulated genon — of the haploid cell cycle (see below). The dipcellulogyre models the replicated $\left(C^{4 \mathrm{D}}\right.$; gyrapex) and pre-replicated $\left(C^{2 \mathrm{D}}\right.$; gyrobase) states in multicellular eukaryotes that are not metazoan [669]; the gyradaptor, 2(D, represents the diploid cell cycle $\mathrm{G}_{0}$. In this regard, two nonlinear steps explain the origin of the eukaryotic cell: $(i)$ expansion of subsumed gyrosystems on the digenon (2D) on the hapcellulogyre to a point of critical IEM content elicits tetragenon (4D) formation; (ii) gravitational collapse (attraction by the genon and all inherent gyrosystems) from within the dipcellulogyre shunts the IEM into gyrosystems and complexifies from within to without (endomembrane system (3.4); novel RNA, protein, and DNA architecture (3.5-3.7); mitosomes, hydrogenosomes, and plastids [670,671]) — and a hapcellulogyre emerges nested like a Matrioshka doll within a dipcellulogyre. This is consistent with autogenic hypotheses [672] yet provides an alternate view to mainstream serial endosymbiotic theory $[673,674]$ and the hydrogen hypothesis [675].

The acellulogyre (Figure $2 \mathrm{~h}$ (iii)), the gyrobasal [Ç] models an achromosomal, anucleate, or DNA-lacking cell or cell system, e.g. aneuploidy [676], $\rho^{0}$ mitochondria [677], hydrogenosomes, platelets [678], red blood cell [679], or apoptotic or differentiating cell [680,681]:

$$
\begin{aligned}
& C^{2 \mathrm{D}} \rightarrow 2 \mathbb{D}+[\mathrm{C}] \\
& \left.\mathrm{C}^{4 \mathrm{D}} \rightarrow 4 \mathbb{D}+[C]\right]
\end{aligned}
$$


Cell cycle. The four-dimensional cellulogyre is another way of understanding the three-dimensional cell cycle (cellulocycle): $\mathrm{G}_{0}$ is the singularity; Restriction point, or start [682], is the gyrobase; $\mathrm{G}_{1}$ phase [683], is the transition from gyrobase to hemi-bas-apex (HBA, halfway from the ground to excited state); S phase, or DNA replication [684], is the transition from HBA to gyrapex (the excited state, $C^{4 D}$ and $C^{2 D}$ ); $G_{2}$ phase [685] is the transition from gyrapex to hemi-apica-basal (HAB, halfway from the excited to ground state); $M$ phase [686] and cytokinesis, or cell division [687], is the transition from $\mathrm{HAB}$ to the gyrobase (the ground state, $C^{2 \mathrm{D}}$ and $C^{\mathrm{D}}$ ). The more proximal the cellulon (Figure $2 \mathrm{~h}(i v)$ ) particle is to the genon singularity, the more unstable and rapid the interconversion of these phases, i.e., the oscillation between two extreme gyrostates. This rapid oscillation explains why, for example, the cell cycle in the Drosophila syncytial blastoderm has no gap phases [688,689].

From an evolutionary standpoint, the earliest and most basic cells (archaebacteria, eubacteria) are closest to the genon and thus thermodynamically unstable, with fast division times (20-30 minutes). The more evolutionary recent cells, eukaryotes, are furthest from the genon singularity and take longer to complete their cycles around it (yeasts, $\sim 1.5 \mathrm{~h}$, protozoans, $6-8 \mathrm{~h}$, somatic cells $10-24 \mathrm{~h}$ ). Thus, the cellulogyre models the cyclical nature of the living cell systems and the evolution of these cycles in said systems [690,691].

Cellular Form. One of Haeckel's enduring naturalistic offerings is a magnificent late $19^{\text {th }}$ century view of the numerous forms of life [692]. With the advent of deconvolution, confocal, and two-photon microscopy [693,694], scientists have obtained a rich and detailed catalog of cell form and function to supplement Haeckel's vitascape. Despite this supreme microvision, one cell biologist publicly lamented about the limits of the trade [695]. To salve this concern and to understand the variety of cell forms and functions, I applied the cellulogyre accordingly. For example, the gyrobasal $\left[C^{\mathrm{D}}\right]$ represents a haploid cell polymer, a hapcellulonexus,

$$
\left[C^{\mathrm{D}}\right]=C^{\mathrm{D}}, C^{\mathrm{D}} \mathrm{C}^{\mathrm{D}}, \mathrm{C}^{\mathrm{D}} \mathrm{C}^{\mathrm{D}} \mathrm{C}^{\mathrm{D}} \mathrm{C}^{\mathrm{D}} \cdots
$$

and the gyrobasal $\left[C^{2 \mathrm{D}}\right]$ represents a diploid cell polymer, a dipcellulonexus,

$$
\left[C^{2 \mathrm{D}}\right]=\mathrm{C}^{2 \mathrm{D}}, \mathrm{C}^{2 \mathrm{D}} \mathrm{C}^{2 \mathrm{D}}, \mathrm{C}^{2 \mathrm{D}} \mathrm{C}^{2 \mathrm{D}} \mathrm{C}^{2 \mathrm{D}} \mathrm{C}^{2 \mathrm{D}} \cdots
$$

Hapcellulonexuses and dipcellulonexuses are predicted to be the most basic higher-order organization of the cellulogyre. These nexuses aggregate and fractalize into hap- and dipcellulomatrices. These single cell layer matrices or surfaces are manifest, for example, in leaves [696], microbial mats [697], fruiting bodies [698], or a mere blade of grass. These matrices assemble into higher-order structures as well, as several haploid and diploid algal, fungal, and plant cells cell systems grow and/or exist in gyratory form (cellulohelices; Figure 4h). The oocyte and many cell types are cellulospheres, having the hallmark spherical form that is found throughout nature and a consequence of omnidirectional expansion of the genogyre within the omnidirectionally expanding cellulogyre. Paleobiologically, the acellulogyre models the evolutionary remains of less complex or thermodynamically unstable cellular forms: plant fossils, stromatolites, and fossilized microbial mats [699]. In this regard, acellulons contain the membrane signature of life and the protein networks that hold them together [700] but are predicted to lack their genomes.

The core features of the gyromodel resolve the matter of how cellular shape and size is established and maintained [701,702]. For instance, the relativistically high energy, unstable, excited state of the cell $\left(C^{2 \mathrm{D}}\right.$ and $\left.C^{4 \mathrm{D}}\right)$ is modeled as cell learning, or cellulognosis. The lower energy, stable, ground state 
$\left(C^{\mathrm{D}}\right.$ and $C^{2 \mathrm{D}}$, respectively) is modeled as cellulomnemesis. Theory thus demonstrates that the cell retains all of its evolutionary history embedded within its biomolecules and particles. Precisely mirroring this theory, the modern cell maintains its overall spatiotemporal information by adapting to acute and/or chronic physical, bioenergetic, and pharmacological cues [703-705] that are received, dissipated, and homeostatically integrated in gyrosystems within itself. Other features of the modern cell are noteworthy [706,707].

Aging and Death. Because cell death is modeled by the acellulogyre, aging and death - another unclear and unsolved problem of biological science [708-710] — is clarified. As modeled in this theory of life, a cell divides as long as IEMs flow through and from the subsumed gyrosystems (from the electrogyre to the cellulogyre). In this regard, since the cell is composed of CHNOPS and other chemical elements that ultimately emerge from and is modeled by the electrogyre, this matter gyrates on a grand scale from the electrogyre to the cellulogyre and from the cellulogyre back to the electrogyre. Senescence - the process of cellular deterioration - is modeled as the thermodynamic instability of the cell or cell system due to the gyrosystemic attractive force increasingly countervailing but not surmounting the repulsive force over evolutionary spacetime. Death is a consequence of gravitational collapse of the cellulogyre into its singularity (genon) due to unobstructed attractive force exerted by, on, and within all subsumed gyrosystems. Consistent with this explanation, in death, the undividing cell begins to break down and, without preservation, ultimately recycles its chemical molecules through the biosphere. The biotic death spiral [711] is more than just a play on words; it reflects the unavoidable gravitational collapse of the cellulogyre.

Meiosis and sex. The origin of sex is a nebulous affair, having occurred in the surreptitious record of life's evolution. While there are many ideas related to sexual origins (for example, [712-714]), a common theme is the oscillatory diploid-haploid life cycle between gamete fusion (syngamy) and meiosis [715]. Recall that, in a gyre, a mIEM particle can exist in only one of the three spatiotemporal locations: the excited state, the ground state, or in the singularity itself (2.3.3). With this in mind, the attractive force of the genon on the dipcellulogyre models oogenesis [716,717],

$$
\text { o : }(i) C^{4 \mathrm{D}} \rightarrow(i i) \mathrm{C}^{2 \mathrm{D}}+2 \mathbb{D} \rightarrow(i i i)\left(\mathrm{C}^{\mathrm{D}}+\mathbb{D}\right)+\mathrm{C}^{2 \mathrm{D}} \rightarrow(i v) \mathrm{C}^{\mathrm{D}}+\mathbb{D}+\mathbb{D}+\mathbb{D}
$$

(i) the attractive genon induces the first meiotic division of the primary oocyte $\left(\mathrm{C}^{4 \mathrm{D}}\right)$; $(i i)$ this produces the secondary oocyte $\left(C^{2 D}\right)$ and the first polar body (2D); (iii) the secondary oocyte undergoes the second meiotic division (substituting the hapcellulogyre here), yielding the mature ovum and a polar body (D) and the first polar body expands $\left(\mathrm{C}^{2 \mathrm{D}}\right)$; and, $(i v)$ being that the dipcellulogyre gyrobase is the same as the gyrapex of the hapcellulogyre ( $c f$. Figures $2 \mathrm{~h}(i)$ and $(i i)$ ), both particles gravitationally return to the genonic state $((D)+(D)$. Modeling spermatogenesis, I have,

$$
\widehat{O}:(i) C^{4 \mathrm{D}} \rightarrow\left(\text { ii) } 2 C^{2 \mathrm{D}} \rightarrow(\text { iii }) 4(\mathrm{D}\right.
$$

This flow diagram, in parsed fashion, shows: $(i)$ the primary spermatocyte $\left(C^{4 D}\right)$ undergoes mitotic division (dipcellulogyre); (ii) both cells emerge from the division as secondary spermatocytes $\left(2 \mathrm{C}^{2 \mathrm{D}}\right.$; one is $C^{2 \mathrm{D}}$, other is rapidly $2\left(D \rightarrow C^{2 \mathrm{D}}\right.$ ); (iii) these cells divide, then arrest in $\mathrm{G}_{0}$, the hapcellulogyre singularity $(2 \mathbb{D}+2(\mathbb{D}=4(D)$. 
So, in the end of gametogenesis, the sperm (D) is one mating type or gamete and the egg $\left(C^{D}\right)$ is the other gamete. Modeling sex, the hap- and dipcellulogyres themselves model the manner by which the sperm (genon singularity) fertilizes (exerts a repulsive force) on the egg (gyrobasal cellulon), forming the zygote (lifting it to the high energy state), which ultimately is repelled by DNA replication (digenon expansion) to the highest potential energy state $\left(C^{4 \mathrm{D}}\right)$. Oversimplifying:

$$
\mathrm{C}^{\mathrm{D}}+(\mathrm{D}) \rightarrow \mathrm{C}^{2 \mathrm{D}}+2 \mathbb{D} \rightarrow \mathrm{C}^{4 \mathrm{D}} \text {. }
$$

Summarizing, a single genonic quantum can exert either the attractive force (meiosis) or the repulsive force (sex), but cannot execute both phenomena simultaneously. Note the oscillation between countervailing forces is a corollary to gyraxioms that treat other oscillating features of the gyromodel $\left(G_{V}\right.$ and $\left.G_{V I}\right)$. Importantly, this theory conforms well to the hormonal cycles that drive gametogenesis and the juxtaposition of gametes [718-723]. The genon-as-singularity concept is presented in Figure 3 (i). Understanding the large-scale relationships of hormones within the diploid-haploid life cycle can be considered as Matrioshkagyres (Figures 3e,j). Finally, the cellulogyre and intrinsic gyrosystems are a concrete substitute to Weismannian thinking [724].

$C$-value enigma. The $\mathrm{C}$-value enigma states that less evolutionary developed cell types have greater genome size than more complex cell systems [725]. Solving this enigma requires reviewing the evolutionary trajectory just prior to the emergence of the cellulogyre: the genogyre is the final point of deposition for all of the accumulated IEM subsumed within itself (Figure 5). A consequence of incorporating all of this information, energy, and matter is genogyre expansion, which models genomic expansion (complexification). The closer the genon is to the aminon singularity, the more unstable and simple the genome; the further from the singularity, the greater the complexity. On a macrocosmic (i.e., kingdom-wide, organism-wide) scale, the cellulon expands (complexifies) to accommodate genon expansion within itself.

By way of reminder, just as each particle in a gyrosystem oscillates between unstable and stable states, so too the gyrosystem itself (which should be thought of as a quantum or as one or more particles $\left(\mathrm{G}_{\mathrm{I}}, \mathrm{G}_{\mathrm{III}}\right)$ oscillates between unstable and stable states within another gyrosystem. Whereas the former oscillation is spatiotemporally brief, the latter is more prolonged, as there is more IEM to mobilize. So, then, in complexifying, the cellulon becomes the nascent point of IEM deposition in lieu of the genon. The closer the cellulon particle is to the genon, the more complex the genome and less complex the cell system; the further the cellulon is from the genon, the more complex the cell system and less complex the genome. This dynamic cellulon-genon relationship affords a novel perspective on the C-value enigma that echoes a prior hypothetical solution [726].

Extracellular Material and Integument. Without thermodynamic support of the genon, the cellulon has direct relationships with the aminon and subgyres $\left(\mathrm{G}_{\mathrm{XI}}, \mathrm{G}_{\mathrm{XI}-1}\right)$. Briefly, this thermodynamic shunt helps clarify not only the proteinaceous extracellular matrix that "links" cells together [727,728],

$$
[C]=C_{A}, C_{A} C_{A}, C_{A} C_{A} C_{A} C_{A} \ldots .
$$

but also the integumentary system (e.g., skin, feathers, beaks, scales, hair, shells, hooves, tusks, and claws; [729,730]). The asymmetric chirality of these extracellular structures appear as whorls, helices, or spirals $[731,732]$ and are due to the gyradaptive forces within the cell.

Circadian rhythms. A large number of cells have an internal "clock," a system that is responsible for maintaining periodic oscillations between states of metabolic, physical, and chemosensory activity 
and inactivity [733,734]. These temporal rhythms are called circadian because they occur over a 24-hour period. Although there is compelling evidence that changes in RNA and protein expression levels are associated with changes in these rhythms [735-737] and there are models to make sense of this data [738], there is no unifying theoretical framework.

Applying the ohiogyre frame here helps clarify these rhythms. Recall that a lunar core (an macroxyon with a macroelectron singularity) gyrates around a planetary core (a macroelectron with macrophoton singularity) in an ohiogyre (3.2). Under this scheme, the cell (a cellulon with a genon singularity) is modeled as rotating around a genomic singularity (a genon with an aminon singularity) in an ohiogyre. That is to say, the oscillating cellular particle (one cell in a cell population) orbits around the attractorepulsive genon singularity (quiescent, unreplicating, or post-replicative cells in that population; [739]). Because one cell cannot be understood apart from its cellular heritage and the cell is dependent upon all of the internal and external thermodynamic cues (e.g., ions, nutrients, energy; $\mathrm{G}_{\mathrm{IV}}, \mathrm{G}_{\mathrm{VIII}}$ ), circadian rhythms may be considered only from the history of the cells under examination.

The Self-organization and Self-regulation of Life. This theory concomitantly treats both the microevolution and macroevolution of life. From a microevolutionary standpoint, the gradual changes in individual cell are modeled by the countervailing adaptive forces - attractorepulsion, creatodestruction, expansocontraction - that emerge from within the cellulogyre and can be experimentally observed (e.g., [740]). Moreover, the gyromodel is in lockstep with mainstream thinking regarding the self-organizational properties of cells [741]. On a macroevolutionary scale, the expansion of the universe exerts a thermodynamic repulsive force through the macrogenon with ultimate deposition into the macrocellulogyre; this provides a simple explanation for microbial ontogenesis, phylogenesis, and evolution [742]. Since one cell consumes, retains, and/or expels parts or whole of another cell during phagocytosis [743], endocytosis [744], and endosymbiosis, cell mass is modeled as cycling through a macrocellulogyre.

In conclusion, the cellulogyre demonstrates that while the Latin phrase Omne vivum ex vivo ("all life [is] from life") is true, it is ontically incomplete. What cell theory was unable to explain - how the first cell originated - this theory explains as the turning and churning of information, energy, and matter in a widening gyre.

\section{Conclusions}

I have compiled and unveiled an axiomatic, experimentally testable, empirically consistent, heuristic, and unified theory of life. Given the breadth and depth of this work, I summarize the theoretical organization in two different ways. The first (Figure 5) is a left-to-right schematic that accounts for feedforward and feedback between, among, and within gyrosystems. The second (Figure 6) is a within-to-without schematic that reveals gyre nesting and chiral toggling. These two schemes afford complementary perspectives on how cellular life originates, evolves, exists, and functions.

\subsection{Theoretical Solutions, Limitations, and Expectations}

Although there have been a handful of theories that model the cell and the origin of life [745-750], to the best of my knowledge, I am presenting the first and only scientific theory of life from the quantum to the living cell. On this basis, my theory is sui generis. I broach the correctness of the 
theory, reiterate a handful of original solutions to protracted scientific problems, and discuss several issues related to comprehensiveness. I pepper this subsection with several theoretical predictions.

Solutions. A correct theory should not only explain how things work but explain why things are the way they are. This theory of life is correct-in precise accordance with natural laws and scientific truths. The ergodic gyromodeling of the origin of both the living cell and the biosphere is correct. The evolutionary positioning of celestial bodies, chemical elements, biogeochemical cycles, biomolecules, and genetic material is likewise correct.

Figure 6. Within-to-without theoretical framework. The electrogyre (where $\mathrm{e}^{\gamma}$ denotes all lepton potentialities) is within the oxygyre (where $\mathrm{O}^{\mathrm{e}}$ denotes all oxyon potentialities) which is within the carbogyre (where $\mathrm{C}^{\mathrm{O}}$ denotes all carbyon potentialities) which is within the phosphogyre (where $\mathrm{P}^{\mathrm{C}}$ denotes all phosphon potentialities) which is within the ribogyre (where $\mathrm{R}^{\mathrm{P}}$ denotes all ribon potentialities) which is within the aminogyre (where $\mathrm{A}^{\mathrm{R}}$ denotes all aminon potentialities) which is within the genogyre (where $\mathrm{D}^{\mathrm{A}}$ denotes all genon potentialities) which is within the cellulogyre (where $C^{\mathrm{D}}$ denotes all cellulon potentialities). Matrioshkagyres - nested antichiral gyres - achieve homeostasis by reducing the rate of IEM metabolism and flow between, among, and within gyrosystems. Time flows from within to without: microcosmically, the rate of each cycle decelerates, as an electron cycles much faster than a cell cycles; macrocosmically, the rate of each cycle decelerates, as planetary axial rotation cycle is relativistically faster than the existential cycle of a particular cellular species. Please consider the widening gyre in light of universal expansion.

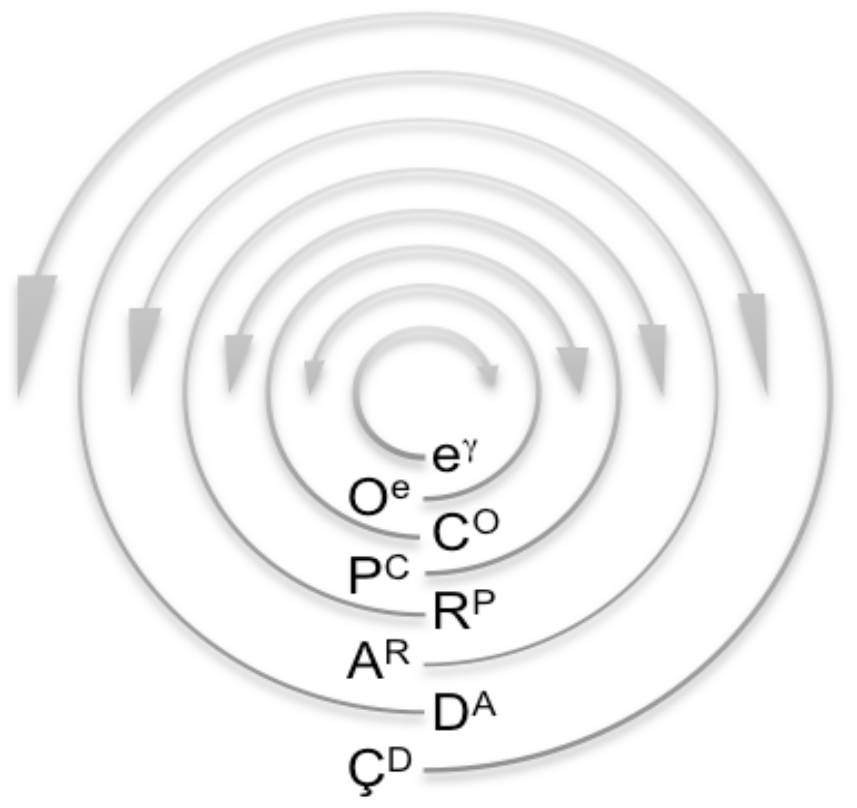

I refer the reader to the Theory section for a complete presentation of theoretical answers to many of science's most challenging questions [751,752]. Here, for brevity, I highlight only one theoretical solution for each gyrosystem. The electrogyre explains quantum gravity, unifying quantum mechanics and general relativity in a frame beyond the standard model [753]; the oxygyre explains the mysterious properties of water [133]; the carbogyre explains the emergence of hydrocarbons in the Earth's mantle 
and crust, resolving the biotic/abiotic petroleum debate [754]; the phosphogyre explains why phosphorus is "life's bottleneck [755]" and the dominant roles of phosphate in biology [756]; the ribogyre solves the problem of novel genetic information [757-759]; the aminogyre explains the origin and nature of the translation apparatus, one of theoretical biology's grand unsolved problems [488,760]; the genogyre clarifies the correct relationship of DNA, protein, and RNA, quelling anonymous protestations against the central dogma [761,762]; and the cellulogyre reveals that life originates in any biosphere wherever the thermodynamics of information, energy, and matter are accommodating, consistent with ideas regarding hierarchical complexification of and in the universe [763]. Together, the theoretical framework confirms what many modern theoretical physicists have proposed: that the classical world is "quantum all the way [764]." The gravity and implications of these solutions are discussed below.

Comprehensiveness. Although I fit the fundamental, structural data from physics, chemistry, and biology, as they are broadly defined, I did not incorporate all of the evidence in those fields. Moreover, I addressed major scientific enigmas, anomalies, and paradoxes but did not address minor puzzles and curiosities. Based upon the successful record of fitting data to the eight presented gyrosystems, I expect more comprehensive data fitting to be profitable and of general interest.

Scope. The theory outlined in this manuscript is limited in scope. I did not provide gyrosystems to model much of the scientific evidence related to astrophysics, particle physics, and cosmology before the electrogyre, nor did I integrate organismal, ecological, and ethological data after the cellulogyre. I predict that further gyromodel application will reveal its explanatory breadth and power. For example, given that complexity theorists find there to be a unifying organization in ecosystems, language, and economics [765-767], I predict the gyromodel will find applications in these subject matters. I also expect the gyromodel to fit data related to the ontogenesis and phylogenesis of Homo sapiens.

\subsection{Laws of Nature}

This theory demonstrates that the complex biology of life obeys known natural laws as they pertain to physics and chemistry. As broadly defined, a law of nature is a physical or scientific principle that is a systematic, general, and formal statement derived from empirical observations of natural phenomena [768,769]. Natural laws have several properties: basic — an essential, necessary, and common foundational principle; universal - apply throughout the visible universe; true, or unfalsifiable - no evidence to contradict its validity; absolute - not subject to conditions or limitations; immutable - unchanging, stable. With this work, I have given incontrovertible proof for the following eight laws of nature, most of which have already been articulated and promoted.

Fourth Law of Thermodynamics. The theoretical framework sheds light on how life maintains order and complexifies in spite of entropy: the repulsive force of the gyradaptive singularity elevates a particle to its excited state, offsetting the effects of it cycling to the ground state. The gyromodel thus confirms the existence of the fourth law of thermodynamics [770], the ordering law of the universe.

Law of Polymers. A key theoretical implication is that the polymeric form is compulsory in all IEM arrangements - as modeled by the secondary and tertiary gyrobases. This is not surprising, as a every known living system requires biomolecular oligomers of a certain length [771]. Thus, the gyromodel reveals a natural law related to IEM organization: a law of polymers. 
Law of Vortex Motion. This theory conclusively demonstrates that all physical systems, particles, and phenomena in the microcosmic and macrocosmic realms obey a vortical trajectory. In so doing, the framework validates the Democritean assertion in the Model section: vortex motion is a natural law.

Law of Correspondence. The theoretical framework shows that biopoiesis - the evolution and origin of life-is recapitulated in any and every extant cell. Furthermore, the electrogyre-in accurately depicting quantum gravity - shows there to be one model that explains the structure, function, and character of both cosmic and atomic phenomena. Hence, in proving correspondence between the macrocosmic and microcosmic realms through all gyrosystems, the Hermetic and Bohrian principle of correspondence [772] is elevated from a philosophical and quantum mechanical principle to a natural law.

Law of Complementarity. Bohr also proposed a complementarity principle-i.e., objects have multiple contradictory properties - to describe, for instance, the wave-particle duality of the quantum. The gyromodel and its axioms elevate this principle to a natural law by proving the complementary necessity of attraction and repulsion, anabolism and catabolism, learning and memory, spacetime position and trajectory, excited and ground states, solids and gases, units and polymers, among other pairs of phenomena.

Law of Relativity. Because the complementary pairs of phenomena, IEM, and states are always relative to any quantum, particle, or gyre of the gyromodel — and hence ever changing - this demonstrates that relativity is not only theory but a law of nature.

Law of Trimergence. Every majorgyre of the theoretical framework emerges with, cycles as, and is sustained and unified by a quantal triad. The triune organization of nature is observable in, for example, the three generations of leptons, three oxygen atoms in carbonic acid, the three phosphates in NTP, three RNAs in protein synthesis, three elemental forms (metals, metalloids, non-metals), three phases of water, triplet genetic code, and IEM. This tri-emergent phenomenon (trimergence) is thus a natural law.

Law of Unity. Each gyrosystem defies simplification; experimental analysis of a gyrosystem in purported isolation indubitably fails to account for sub- or supervenient systems, particles, and processes (Figure 5). The complexity of life thus surreptitiously withheld a law of unity that had been intimated [773,774]. In light of theory and this natural law, although paradoxically reducible to its component parts, a cell is rightfully indivisible, a unity with and in the evolving universe. This natural law decrees that physical reality is one.

A foundational goal of science is to identify and understand the physical laws that govern the visible universe [775,776]. The demonstration of new laws of nature is thus an important scientific achievement.

\subsection{Theoretical Proofs and Implications}

I have arrived at several compelling proofs from this theory of life; on this matter, proof is defined as extraordinary evidence that establishes a fact or the truth of a statement. In this section, I detail theoretical proofs related to origins, time, order, adaptation, evolutionary emergence, and life on Earth and in the universe. I conclude this subsection with one point regarding metaphysics, another regarding 
causality and necessity, and another on the relationship of this theory to the epistemological progress of science.

Origins. In this theoretical study, I have demonstrated that each gyrosystem singularity represents the origin of that gyrosystem. In other words, the singularity is the beginning and the end, the thermodynamic source and the sink of each cycle of IEM through a gyre. Because the dwell time in each singularity is immeasurable - as each singularity is potentially infinite - it has been an empirical challenge to recognize its existence without this theory. The theoretical model harmonizes with Cantor set theory [777,778] by demonstrating that the origin of life is a consequence of iterative nested origins or singularities (Figures 3e,i, and 6), which, despite relying on the same core model, are paradoxically more than one kind of infinity.

Arrow of Time. Eddington equated entropy with "the arrow of time" because of time's asymmetry in observable physical processes and in evolution [779]. Theory accurately depicts the vectorial nature of time as IEM flow from within the electron to the cell as nested sets of singularities (Figure 6). Macrocosmically, time progresses from Earth's origin (4.6 billion years ago) to the origin of the first living cell ( $\sim 3.4$ bya, the fossil record of cellular life [780]). Microcosmically, from electron cycling to cell division, time decelerates from $\sim 10^{-8}$ seconds to 90 minutes (doubling of typical yeast cell).

Order and Disorder. Given the law of relativity, IEM order and disorder are demonstrated to be relative to the singularity. Further, given the law of complementarity, universal order and disorder paradoxically co-exist. In proving this contradictory fact, my theory does not "collapse in deepest humiliation [781]," but rather reflects and honors the true nature of the physical world.

Adaptation. This theory explains that all adaptation is the emergent, cumulative, and ongoing learning and memory of oscillating gyrosystem particles due to the attractorepulsive, expansocontractive, and/or creatodestructive force(s) of the quantal singularity. Because every gyrosystem adapts, this explains how and why physical, chemical, molecular, and cellular systems always seek homeostasis [782,783].

Evolutionary emergence. The Darwinian theory of evolution by natural selection does not address how novelty emerges in the universe [784,785]. As modeled here and as observable in the natural world, gyres expand and develop as a consequence of continual IEM flow, rearrangement, and coalescence. When the maximum carrying capacity of a gyrosystem is reached, it extrudes IEM due to spatiotemporal constraints imparted by sub- and supragyrosystems; this models self-organized criticality [786]. Gyrosystem collapse thus converts the accumulated, unsustainable, potential IEM into kinetic IEM, eliciting the emergence of an a priori unpredictable organization that is more thermodynamically stable. Written another way, theory shows that the disassembled gyrosystem provides the architectural basis and thermodynamic driving force for evolutionary complexification. This theory supports what Gould and Eldredge implied in their theory of punctuated equilibrium [787]: evolutionary emergence occurs by a true quantal leap. I conclude that my theory is a comprehensive and scientifically accurate alternative to natural selection.

Meaning of Life. Life has many definitional meanings but lacks a complete and consistent scientific explanation. In this work, I have pursued and arrived at a scientific answer to the Schrödingerian question, "What is Life? [1]." Traditionally, the living cell is commonly called "animate" and all other biospheric and cellular chemicals and molecules are called "inanimate." However, this theory and the law of vortex motion prove that all these physical systems gyrate and are, as such, "animated." 
Moreover, theory-defined laws of unity and correspondence require that life and Earth evolve as one, with thermodynamically appropriate conditions (the fitness of the biosphere [788,789]). Unexpectedly, then, this theory reveals that Earth — or, for that matter, any celestial, physical, chemical, and molecular system - is alive, that is, synonymous with life. Given this definitional and conceptual upheaval, I propose that a very open and candid discussion of the meaning of life-well beyond this text - is in order. On this topic, it may be useful to consider how scientifically redefining life elucidates non-scientific, eudaemonic meanings of "life," "living," or "alive," related to ontology, consciousness, sentience, behavior, vocation, or social interactions.

Search for Extraterrestrial Life. The quest to discover extraterrestrial forms of life in the universe is predicated on a set of definitions and assumptions of what life is [790]. Furthermore, astrobiologists and exobiologists seek an understanding of the conditions for habitability and distribution of life on other planets and throughout the cosmos [791-793]. In addition to modeling terrestrial, or Earth-bound, life, this general theory models exterrestrial forms of life. Indeed, this theory not only predicts but also proves that the universe is teeming with life - a result of omnidirectional universal evolution.

Causality, Chance, and Necessity. In the second half of the $20^{\text {th }}$ century, Monod painted a Weltanschauung in which life was happenstance, stochastic, and largely without purpose [794]. While this worldview may appeal to some, it was and is premature to conclude that it is the correct perspective of life. With this theory, I show that any measurement of the physical living system changes the system, causing it to evolve; any calculation or prediction excludes information. As gyrosystems model both the cause of a phenomenon and the effect of that phenomenon, it is accurate to write that the gyrosystem is the phenomenon itself. Therefore, cellular life is not chance, but necessity - by virtue of theoretically validated natural laws and of theoretically unified phenomena that have occurred in the evolutionary history of the universe and are occurring now, at every Cartesian coordinate throughout the universe.

Metaphysics. The gyre models the living universe perfectly. I have been unable to find one system, particle, event, or process - at any point or stage leading up to or during the origin of life - that does not consent to modeling onto the gyre form. In other words, there is no "before" or "after" the gyre in a spacetime sense; the gyre is evolutionarily and existentially omnipresent. This theory proves that the gyre is the long-sought invisible and inevitable metaphysical element of the universe, fulfilling a philosophical goal that dates to ancient Greece [795].

Epistemological rupture. The philosopher Bachelard claimed that scientific history is replete with unconsciously constructed or immanent "epistemological obstacles," that are eventually broken through and shed during "epistemological rupture [796]." I conclude that my theoretical work elicits a Bachelardian rupture of intradisciplinary noöspheres and interdisciplinary boundaries. Kuhn proposed a related concept of "paradigm shift" to explain the process surrounding worldview conversion during a scientific revolution [797]. Whether the advent of this theory elicits a Kuhnian gestalt switch is debatable, though such an iconoclastic event has been foretold [798-800].

\subsection{Concluding Remarks}

In science and theory, the principle of parsimony dictates that the most straightforward, plain, and frugal model of an observation or set thereof is more favorable and likely right. As my theoretical 
framework coalesces a vast amount of accumulated scientific evidence into one neat, lawful, and interconnected modular structure, it abides by this principle. In conclusion, this catholic theory provides an innovative and elegant solution to the origin, evolution, and nature of life in the cosmos. I humbly proffer my theory as a viable system for knowing life.

\section{Acknowledgments}

I thank all who have read or commented on my manuscript. I acknowledge the innumerable scientists, theoreticians, mathematicians, philosophers, and sages who contributed to unraveling the mystery of life. No funding source supported this work.

\section{References and Notes}

1. Schrödinger, E. What is Life? The Physical Aspect of the Living Cell; With, Mind And Matter; \& Autobiographical Sketches; Cambridge University Press: Cambridge; New York, NY, USA, 1992; p.184.

2. Crick, F. Life Itself: Its Origin and Nature; Simon and Schuster: New York, NY, USA, 1981; p. 192.

3. Hoyle, F.; Wickramasinghe, N.C. Our Place in the Cosmos: The Unfinished Revolution; J.M. Dent: London, UK, 1993; p. 190.

4. Oparin, A.I.; Morgulis, S. The Origin of Life, 2nd ed.; Dover Publications: Mineola, New York, NY, USA, 2003; p. 270.

5. Bernal, J.D. The Origin of Life; World Publishing Co.: Cleveland, OH, USA, 1967; p. 345.

6. Wachtershauser, G. On the chemistry and evolution of the pioneer organism. Chem. Biodivers. 2007, 4, 584-602.

7. Gesteland, R.F.; Cech, T.; Atkins, J.F. The RNA World: The Nature of Modern RNA Suggests a Prebiotic RNA, 2nd ed.; Cold Spring Harbor Laboratory Press: Cold Spring Harbor, NY, USA, 1999; p. 709.

8. Gesteland, R.F.; Atkins, J.F. The RNA World: The Nature of Modern RNA Suggests a Prebiotic RNA World; Cold Spring Harbor Laboratory Press: Cold Spring Harbor, NY, USA, 1993; p. 630.

9. Gesteland, R.F.; Cech, T.; Atkins, J.F. The RNA World: The Nature of Modern RNA Suggests a Prebiotic RNA World, 3rd ed.; Cold Spring Harbor Laboratory Press: Cold Spring Harbor, NY, USA, 2006; p. 768.

10. Agar, W.E. A Contribution to the Theory of the Living Organism; Melbourne University Press in Association with Oxford University Press: Victoria, Australia, 1943; p. 207.

11. Cannon, H.G. The Evolution of Living Things; Manchester University Press: Manchester, UK, 1959; p. 152.

12. De Duve, C. Blueprint for a Cell: The Nature and Origin of Life; N. Patterson: Burlington, NC, USA, 1991; p. 275.

13. Eigen, M.; Winkler, R. Steps Towards Life: A Perspective on Evolution; Oxford University Press: Oxford; New York, NY, USA, 1992; p. 173. 
14. Elsasser, W.M. Reflections on a Theory of Organisms: Holism in Biology; Published for the Johns Hopkins Dept. of Earth and Planetary Sciences by the Johns Hopkins University Press: Baltimore, MD, USA, 1998; p. 160.

15. Fry, I. The Emergence of Life on Earth: A Historical and Scientific Overview; Rutgers University Press: New Brunswick, NJ, USA, 2000; p. 327.

16. Ho, M.-W. The Rainbow and the Worm: The Physics of Organisms; World Scientific: Singapore and River Edge, NJ, USA, 1993; p. 202.

17. Jacob, F. The Logic of Life: A History of Heredity; Princeton University Press: Princeton, NJ, USA, 1993; p. 348.

18. Küppers, B.-O. Information and the Origin of Life; MIT Press: Cambridge, MA, USA, 1990; p. 215.

19. Lahav, N. Biogenesis: Theories of Life's Origin. Oxford University Press: New York, NY, USA, 1999; p. 349.

20. Lillie, R.S. General Biology and Philosophy of Organism; University of Chicago Press: Chicago, IL, USA, 1945; p. 215.

21. Maynard Smith, J.; Szathmáry, E. The Major Transitions in Evolution; W.H. Freeman Spektrum: Oxford, UK and New York, NY, USA, 1995; p. 346.

22. Weiss, P.A. The Science of Life: The Living System-A System for Living; Futura Publishing Co.: Mount Kisco, NY, USA, 1973; p. 137.

23. Zubay, G.L. Origins of Life on the Earth and in the Cosmos, 2nd ed.; Academic Press: San Diego, CA, USA, 2000; p. 564.

24. Davies, P.C.W. The Fifth Miracle: The Search for the Origin and Meaning of Life; Simon \& Schuster: New York, NY, USA, 1999; p. 304.

25. Hargittai, I.; Pickover, C.A. Spiral Symmetry; World Scientific: Singapore and Teaneck, NJ, USA, 1992; p. 449.

26. Ball, P. The Self-Made Tapestry: Pattern Formation in Nature; Oxford University Press: Oxford, UK and New York, NY, USA, 1999; p. 287.

27. Ginzburg, V.B.; Ginzburg, T.V. Prime Elements of Ordinary Matter, Dark Matter \& Dark Energy: Beyond Standard Model \& String Theory, 2nd ed.; Universal Publishers: Boca Raton, FL, USA, 2007; p. 434.

28. Thompson, D.A.W. On Growth and Form; Dover: New York, NY, USA, 1992; p. 1116.

29. Bojowald, M. Singularities and quantum gravity. Cosmol. Gravit. 2007, 910, 294-333.

30. Chrusciel, P.T. Black holes. Conform. Struct. Space Time 2002, 604, 61-102.

31. De Duve, C. Singularities: Landmarks on the Pathways of Life; Cambridge University Press: Cambridge, UK and New York, NY, USA, 2005; p. 258.

32. Blackmond, D.G. The origin of biological homochirality. Cold Spring Harbor Perspect. Biol. 2010, 2, a002147.

33. Jantsch, E. The Self-Organizing Universe: Scientific and Human Implications of the Emerging Paradigm of Evolution, 1st ed.; Pergamon Press: Oxford; New York, NY, USA, 1980; p. 343.

34. Babloyantz, A. Molecules, Dynamics, and Life: An Introduction to Self-Organization of Matter; Wiley: New York, NY, USA, 1986; p. 345. 
35. Solé, R.V.; Bascompte, J. Self-Organization in Complex Ecosystems; Princeton University Press: Princeton, NJ, USA, 2006; p. 373.

36. Feltz, B.; Crommelinck, M.; Goujon, P. Self-Organization and Emergence in Life Sciences; Springer: Dordrecht, The Netherlands, 2006; p. 360.

37. McCabe, G. The non-unique universe. Found. Phys. 2010, 40, 629-637.

38. Gödel, K. On Formally Undecidable Propositions of Principia Mathematica and Related Systems; Dover: Mineola, NY, USA, 1962.

39. Hogan, D.A. Talking to themselves: Autoregulation and quorum sensing in fungi. Eukaryot. Cell 2006, 5, 613-619.

40. Tappy, L.; Chiolero, R.; Berger, M. Autoregulation of glucose production in health and disease. Curr. Opin. Clin. Metab. Care 1999, 2, 161-164.

41. Bateman, E. Autoregulation of eukaryotic transcription factors. Progr. Nucleic Acid Res. Mol. Biol. 1998, 60, 133-168.

42. Hajdukovic, D.S. Dark energy, antimatter gravity and geometry of the Universe. Astrophys. Space Sci. 2010, 330, 1-5.

43. Bacinich, E.J.; Kriz, T.A. The arrow of time in an expanding 3-sphere. Phys. Essays 1999, 12, $80-91$.

44. Reid, R.G.B. Biological Emergences: Evolution by Natural Experiment; MIT Press: Cambridge, MA, USA, 2007; p. 517.

45. Bak, P.; Paczuski, M. Complexity, contingency, and criticality. Proc. Natl. Acad. Sci. USA 1995, 92, 6689-6696.

46. Von Bertalanffy, L. General System Theory; Foundations, Development, Applications; G. Braziller: New York, NY, USA, 1969; p. 289.

47. Kauffman, S.A. The Origins of Order: Self-Organization and Selection in Evolution; Oxford University Press: New York, NY, USA, 1993; p. 709.

48. Bird, R.J. Chaos and Life: Complexity and Order in Evolution and Thought; Columbia University Press: New York, NY, USA, 2003; p. 322.

49. Yockey, H.P. Information Theory, Evolution, and the Origin of Life; Cambridge University Press: New York, NY, USA, 2005; p. 259.

50. Laszlo, E. The Systems View of the World: A Holistic Vision for Our Time; Hampton Press: Cresskill, NJ, USA, 1996; p. 103.

51. Lorenz, E.N. The Essence of Chaos; University of Washington Press: Seattle, DC, USA, 1993; p. 227.

52. Standish, R.K. Why Occam's razor. Found. Phys. Lett. 2004, 17, 255-266.

53. Anderson, P.W. More is different. Science 1997, 177, 393-396.

54. Umpleby, S.A. Physical relationships among matter, energy and information (Reprinted form Cybernetics and Systems '04, 2004). Syst. Res. Behav. Sci. 2007, 24, 369-372.

55. Smil, V. Energies: An Illustrated Guide to the Biosphere and Civilization; MIT Press: Cambridge, MA, USA, 1999; p. 210.

56. Wrigglesworth, J.M. Energy and Life; Taylor \& Francis: London, UK and Bristol, PA, USA, 1997; p. xi. 
57. Lehninger, A.L. Bioenergetics; the Molecular Basis of Biological Energy Transformations, 2nd ed.; W.A. Benjamin: Menlo Park, CA, USA, 1971; p. 245.

58. Von Foerster, H. Understanding Understanding: Essays on Cybernetics and Cognition; Springer: New York, NY, USA, 2003; p. 362.

59. Dakos, V.; Kefi, S.; Rietkerk, M.; van Nes, E.H.; Scheffer, M. Slowing down in spatially patterned ecosystems at the brink of collapse. Am. Nat. 2011, 177, E153-E166.

60. Devreotes, P. Dictyostelium discoideum: A model system for cell-cell interactions in development. Science 1989, 245, 1054-1058.

61. Kuznetsov, E.A. Wave collapse in plasmas and fluids. Chaos 1996, 6, 381-390.

62. Anraku, Y. Bacterial electron transport chains. Annu. Rev. Biochem. 1988, 57, 101-132.

63. Kovacic, P.; Pozos, R.S. Bioelectronome. Integrated approach to receptor chemistry, radicals, electrochemistry, cell signaling, and physiological effects based on electron transfer. J. Recept. Signal Transduct. Res. 2007, 27, 261-294.

64. Trevors, J.T.; Masson, L. Quantum Microbiology. Curr. Issues Mol. Biol. 2011, 13, 43-49.

65. Smolin, L. A crisis in fundamental physics. Update, N. Y. Acad. Sci. Mag. January/February 2006, p. 10-14.

66. Nagel, S. Physics in crisis. Phys. Today 2002, 55, 55-57.

67. Heller, M.; Pysiak, L.; Sasin, W. Fundamental Problems in the unification of physics. Found. Phys. 2011, 41, 905-918.

68. Nikolic, H. Quantum mechanics: Myths and facts. Found. Phys. 2007, 37, 1563-1611.

69. Turyshev, S.G. Experimental Tests of General Relativity. Annu. Rev. Nuclear Part. Sci. 2008, 58, 207-248.

70. Penrose, R. The Road to Reality: A Complete Guide to the Laws of the Universe, 1st Vintage Books ed; Vintage Books: New York, NY, USA, 2007; p. 1099.

71. Lyons, L. An introduction to the possible substructure of quarks and leptons. Progr. Part. Nuclear Phys. 1983, 10, 227-304.

72. Hegstrom, R.A. Electron Chirality. Theochem J. Mol. Struct. 1991, 78, 17-21.

73. Hegstrom, R.A. Weak neutral current and beta-radiolysis effects on the origin of biomolecular chirality. Nature 1985, 315, 749-750.

74. Bryman, D. Three generations of quarks and leptons: Who ordered that? Intersect. Part. Nuclear Phys. 2000, 549, 150-163.

75. Arunan, E.; Raghavendra, B. Unpaired and sigma bond electrons as $\mathrm{H}, \mathrm{Cl}$, and $\mathrm{Li}$ bond acceptors: An anomalous one-electron blue-shifting chlorine bond. J. Phys. Chem. A 2007, 111, 9699-9706.

76. Haviland, D.B.; Delsing, P. Cooper-pair charge solitons: The electrodynamics of localized charge in a superconductor. Phys. Rev. B 1996, 54, R6857-R6860.

77. Reiher, M.; Eickerling, G. The shell structure of atoms. J. Chem. Theory Comput. 2008, 4, 286-296.

78. Williams, R.J.P. The fundamental nature of life as a chemical system: The part played by inorganic elements. J. Inorg. Biochem. 2002, 88, 241-250.

79. Williams, R.J.P.; da Silva, J.J.R.F. Evolution was chemically constrained. J. Theor. Biol. 2003, 220, 323-343. 
80. Truscott, A.G.; Strecker, K.E.; McAlexander, W.I.; Partridge, G.B.; Hulet, R.G. Observation of Fermi pressure in a gas of trapped atoms. Science 2001, 291, 2570-2572.

81. Gensemer, S.D.; Jin, D.S. Transition from collisionless to hydrodynamic behavior in an ultracold Fermi gas. Phys. Rev. Lett. 2001, 87, doi:10.1103/PhysRevLett.87.173201.

82. Anderson, P.W.; Casey, P.A. Hidden Fermi liquid; the moral: A good effective low-energy theory is worth all of Monte Carlo with Las Vegas thrown in. J. Phys. Condens. Matter 2010, 22, doi:10.1088/0953-8984/22/16/164201.

83. Mook, H.A.; Dai, P.; Dogan, F.; Hunt, R.D. One-dimensional nature of the magnetic fluctuations in YBa2Cu3O6.6. Nature 2000, 404, 729-731.

84. Sharma, R.P.; Ogale, S.B.; Zhang, Z.H.; Liu, J.R.; Chu, W.K.; Veal, B.; Paulikas, A.; Zheng, H.; Venkatesan, T. Phase transitions in the incoherent lattice fluctuations in $\mathrm{YBa} 2 \mathrm{Cu} 3 \mathrm{O}$ (7-delta). Nature 2000, 404, 736-740.

85. Zwierlein, M.W.; Abo-Shaeer, J.R.; Schirotzek, A.; Schunck, C.H.; Ketterle, W. Vortices and superfluidity in a strongly interacting Fermi gas. Nature 2005, 435, 1047-1051.

86. Galitski, V.M.; Refael, G.; Fisher, M.P.; Senthil, T. Vortices and quasiparticles near the superconductor-insulator transition in thin films. Phys. Rev. Lett. 2005, 95, doi:10.1103/ PhysRevLett.95.077002.

87. Kopnin, N.B. Vortex dynamics and mutual friction in superconductors and Fermi superfluids. Rep. Prog. Phys. 2002, 65, 1633-1678.

88. Carati, A.; Galgani, L.; Giorgilli, A. The Fermi-Pasta-Ulam problem as a challenge for the foundations of physics. Chaos 2005, 15, doi:10.1063/1.1861264.

89. Moreover, given that the electrogyre is a cyclical, periodic system in which all particles within the gyre continually adapt to one another through photon mobilization and storage, this provides a novel solution to the Fermi-Pasta-Ulam problem.

90. Kalmus, P.I.P. The forces of nature. Interdiscip. Sci. Rev. 1993, 18, 343-349.

91. The frequently presented $2 \mathrm{D}$ lengthening wavelengths of the electromagnetic spectrum from gamma rays to radio rays are modeled as the 4D expansion or widening of the electrogyre from the photonic singularity.

92. Uman, M.A.; Krider, E.P. A review of natural lightning-Experimental-data and modeling. IEEE Trans. Electromagn. Compat. 1982, 24, 79-112.

93. Rowland, H.L. Theories and simulations of elves, sprites and blue jets. J. Atmos. Solar Terr. Phys. 1998, 60, 831-844.

94. The electromagnetic spectral signature of the photonic threshold effect is observed in the colored pre-lightning emissions called red sprites and blue jets.

95. Stenhoff, M. Ball Lightning: An Unsolved Problem in Atmospheric Physics; Kluwer Academic: New York, NY, USA, 1999; p. 349.

96. Abrahamson, J.; Dinniss, J. Ball lightning caused by oxidation of nanoparticle networks from normal lightning strikes on soil. Nature 2000, 403, 519-521.

97. Bohr, N. On the notions of causality and complementarity. Science 1950, 111, 51-54.

98. Pearle, P.; Collett, B. Wavefunction collapse and random walk. Found. Phys. 2003, 33, 1495-1541.

99. Karakostas, V.; Dickson, M. Decoherence in unorthodox formulations of quantum-mechanics. Synthese 1995, 102, 61-97. 
100. Busch, P.; Heinonen, T.; Lahti, P. Heisenberg's uncertainty principle. Phys. Rep. Rev. Sect. Phys. Lett. 2007, 452, 155-176.

101. Marco, R.; Diaz, C.; Benguria, A.; Mateos, J.; Mas, J.; de Juan, E. The role of gravity in the evolutionary emergence of multicellular complexity: Microgravity effects on arthropod development and aging. Adv. Space Res. 1999, 23, 2075-2082.

102. Dubinin, N.P.; Vaulina, E.N. The evolutionary role of gravity. Life Sci. Space Res. 1976, 14, 47-55.

103. Yoshino, T.; Walter, M.J.; Katsura, T. Core formation in planetesimals triggered by permeable flow. Nature 2003, 422, 154-157.

104. Stevenson, D.J. Models of the Earth's Core. Science 1981, 214, 611-619.

105. Taylor, S.R. The origin of the earth. AGSO J. Aust. Geol. Geophys. 1997, 17, 27-31.

106. Forte, A.M.; Mitrovica, J.X. A resonance in the Earth's obliquity and precession over the past 20 Myr driven by mantle convection. Nature 1997, 390, 676-680.

107. Zhang, Y.Z.; Luo, J.; Nie, Y.X. Gravitational effects of rotating bodies. Mod. Phys. Lett. A 2001, 16, 789-794.

108. Sokoloff, D.D. Geodynamo and models of geomagnetic field generation: A review. Geomagn. Aeron. 2004, 44, 533-542.

109. Buffett, B.A. Earth's core and the geodynamo. Science 2000, 288, 2007-2012.

110. Sreenivasan, B. Modelling the geodynamo: Progress and challenges. Curr. Sci. 2010, 99, 1739-1750.

111. Olson, P.; Amit, H. Changes in earth's dipole. Die Naturwissenschaften 2006, 93, 519-542.

112. Greff-Lefftz, M.; Legros, H. Core rotational dynamics and geological events. Science 1999, 286, 1707-1709.

113. Courtillot, V.; Besse, J. Magnetic field reversals, polar wander, and core-mantle coupling. Science 1987, 237, 1140-1147.

114. Crary, F.J.; Clarke, J.T.; Dougherty, M.K.; Hanlon, P.G.; Hansen, K.C.; Steinberg, J.T.; Barraclough, B.L.; Coates, A.J.; Gerard, J.C.; Grodent, D.; et al. Solar wind dynamic pressure and electric field as the main factors controlling Saturn's aurorae. Nature 2005, 433, 720-722.

115. Smith, E.J.; Davis, L., Jr.; Jones, D.E.; Colburn, D.S.; Coleman, P.J., Jr.; Dyal, P.; Sonett, C.P. Magnetic field of jupiter and its interaction with the solar wind. Science 1974, 183, 305-306.

116. Tarduno, J.A.; Cottrell, R.D.; Watkeys, M.K.; Hofmann, A.; Doubrovine, P.V.; Mamajek, E.E.; Liu, D.; Sibeck, D.G.; Neukirch, L.P.; Usui, Y. Geodynamo, solar wind, and magnetopause 3.4 to 3.45 billion years ago. Science 2010, 327, 1238-1240.

117. Khodachenko, M.L.; Ribas, I.; Lammer, H.; Griessmeier, J.M.; Leitner, M.; Selsis, F.; Eiroa, C.; Hanslmeier, A.; Biernat, H.K.; Farrugia, C.J.; et al. Coronal mass ejection (CME) activity of low mass M stars as an important factor for the habitability of terrestrial exoplanets. I. CME impact on expected magnetospheres of Earth-like exoplanets in close-in habitable zones. Astrobiology 2007, 7, 167-184.

118. Howard, T. Coronal Mass Ejections: An Introduction; Springer: New York, NY, USA, 2011.

119. Cho, A. Particle physics. Hints of greater matter-antimatter asymmetry challenge theorists. Science 2010, 328, 1087.

120. Ellis, J. Particle physics: Antimatter matters. Nature 2003, 424, 631-634. 
121. Buser, R. The formation and early evolution of the Milky Way galaxy. Science 2000, 287, 69-74.

122. Alibert, Y.; Broeg, C.; Benz, W.; Wuchterl, G.; Grasset, O.; Sotin, C.; Eiroa, C.; Henning, T.; Herbst, T.; Kaltenegger, L.; et al. Origin and formation of planetary systems. Astrobiology 2010 , 10, 19-32.

123. Craig, N.C.; Gislason, E.A. First law of thermodynamics; Irreversible and reversible processes. $J$. Chem. Educ. 2002, 79, 193-200.

124. Livio, M. The Accelerating Universe: Infinite Expansion, the Cosmological Constant, and the Beauty of the Cosmos; Wiley: New York, NY, USA, 2000; p. 274.

125. Guth, A.H. The Inflationary Universe: The Quest for a New Theory of Cosmic Origins; Addison-Wesley Publishing: Reading, MA, USA, 1997; p. 358.

126. Peebles, P.J.E.; Ratra, B. The cosmological constant and dark energy. Rev. Mod. Phys. 2003, 75, 559-606.

127. Frieman, J.A.; Turner, M.S.; Huterer, D. Dark energy and the accelerating universe. Annu. Rev. Astron. Astrophys. 2008, 46, 385-432.

128. Freese, K. Review of observational evidence for dark matter in the universe and in upcoming searches for dark stars. Cral Ipnl 2009, 36, 113-126.

129. Morgan, C.L. Emergent Evolution; the Gifford Lectures; Williams and Norgate: London, UK, $1923 ;$ p. 313.

130. Bergson, H. Creative Evolution; University Press of America: Lanham, MD, USA, 1984; p. 407..

131. Koonin, E.V. The Biological Big Bang model for the major transitions in evolution. Biology Direct 2007, 2, 21-27.

132. Brack, A. Liquid water and the origin of life. Orig. Life Evol. Biosph. 1993, 23, 3-10.

133. Ball, P. Water: Water-An enduring mystery. Nature 2008, 452, 291-292.

134. Pollack, G.H.; Figueroa, X.; Zhao, Q. Molecules, water, and radiant energy: New clues for the origin of life. Int. J. Mol. Sci. 2009, 10, 1419-1429.

135. Robert, F. Isotope geochemistry. The origin of water on earth. Science 2001, 293, 1056-1058.

136. Truskett, T.M.; Dill, K.A. A simple analytical model of water. Biophys. Chem. 2003, 105, 449-459.

137. Ben-Naim, A. Molecular Theory of Water and Aqueous Solutions; World Scientific: Singapore and Hackensack, NJ, USA, 2009; p. 629.

138. Zachariassen, K.E.; Kristiansen, E. Ice nucleation and antinucleation in nature. Cryobiology 2000, 41, 257-279.

139. Dobretsov, N.L. On the early evolutionary stage of the geosphere and biosphere and the problem of early glaciations. Paleontol. J. 2010, 44, 827-838.

140. Van Dishoeck, E.F.; Blake, G.A. Chemical evolution of star-forming regions. Annu. Rev. Astron. Astrophys. 1998, 36, 317-368.

141. Johari, G.P.; Hallbrucker, A.; Mayer, E. Two calorimetrically distinct states of liquid water below 150 kelvin. Science 1996, 273, 90-92.

142. Pal, S.; Sankaran, N.B.; Samanta, A. Structure of a self-assembled chain of water molecules in a crystal host. Angew. Chem. 2003, 42, 1741-1743.

143. Goldblatt, C.; Zahnle, K.J. Faint young Sun paradox remains. Nature 2011, 474, E3-E4. 
144. Rosing, M.T.; Bird, D.K.; Sleep, N.H.; Bjerrum, C.J. No climate paradox under the faint early Sun. Nature 2010, 464, 744-U117.

145. Jacobsen, S.D.; van der Lee, S.F.M. Earth's Deep Water Cycle; American Geophysical Union: Washington, DC, USA, 2006; p. 313.

146. Hellevang, H. On the forcing mechanism for the H(2)-driven deep biosphere. Int. J. Astrobiol. 2008, 7, 157-167.

147. Oze, C.; Sharma, M. Serpentinization and the inorganic synthesis of H-2 in planetary surfaces. Icarus 2007, 186, 557-561.

148. Pierrehumbert, R.; Gaidos, E. Hydrogen greenhouse planets beyond the habitable zone. Astrophys. J. Lett. 2011, doi:10.1088/2041-8205/734/1/L13.

149. Tian, F.; Toon, O.B.; Pavlov, A.A.; de Sterck, H. A hydrogen-rich early Earth atmosphere. Science 2005, 308, 1014-1017.

150. Zakharov, V.V.; Brodskaya, E.N.; Laaksonen, A. Surface tension of water droplets: A molecular dynamics study of model and size dependencies. J. Chem. Phys. 1997, 107, 10675-10683.

151. Claussen, W.F. Surface tension and surface structure of water. Science 1967, 156, 1226-1227.

152. Stokes, G. On the theory of oscillatory waves. Trans. Camb. Phil. Soc. 1847, 8, 441-455.

153. Constantin, A.; Strauss, W. Rotational steady water waves near stagnation. Philos. Trans. Ser. A 2007, 365, 2227-2239.

154. Poitevin, B. The continuing mystery of the Memory of Water. Homeopathy 2008, 97, 39-41.

155. Davenas, E.; Beauvais, F.; Amara, J.; Oberbaum, M.; Robinzon, B.; Miadonna, A.; Tedeschi, A.; Pomeranz, B.; Fortner, P.; Belon, P.; et al. Human basophil de-granulation triggered by very dilute antiserum against ige. Nature 1988, 333, 816-818.

156. Vega, C.; Conde, M.M.; McBride, C.; Abascal, J.L.; Noya, E.G.; Ramirez, R.; Sese, L.M. Heat capacity of water: A signature of nuclear quantum effects. J. Chem. Phys. 2010, 132, doi:10.1063/1.3298879.

157. Wyrtki, K.; Wenzel, J. Possible gyre gyre interaction in the Pacific-Ocean. Nature 1984, 309, 538-540.

158. Rypina, I.I.; Pratt, L.J.; Lozier, M.S. Near-surface transport pathways in the north atlantic ocean: Looking for throughput from the subtropical to the subpolar gyre. J. Phys. Oceanogr. 2011, 41, 911-925.

159. Zeng, X.C.; Bai, J.E.; Wang, J. Multiwalled ice helixes and ice nanotubes. Proc. Natl. Acad. Sci. USA 2006, 103, 19664-19667.

160. Dismukes, G.C.; Klimov, V.V.; Baranov, S.V.; Kozlov, Y.N.; DasGupta, J.; Tyryshkin, A. The origin of atmospheric oxygen on Earth: The innovation of oxygenic photosynthesis. Proc. Natl. Acad. Sci. USA 2001, 98, 2170-2175.

161. Schafer, G. How did the Earth's oxygen atmosphere originate? Anasthesiol. Intensivmmed. Notfallmedizin Schmerzther. 2004, 39, S19-S27.

162. Thuillier, G. The Sun-Earth relationship. C. R. Acad. Sci. Ser. II 2001, 333, 311-328.

163. Falkowski, P.G.; Godfrey, L.V. Electrons, life and the evolution of Earth's oxygen cycle. Philos. Trans. R. Soc. Lond. Ser. B 2008, 363, 2705-2716.

164. Wayne, R.P. Atmospheric chemistry-The evolution of our atmosphere. J. Phothchem. Photobiol. A 1992, 62, 379-396. 
165. Hyde, W.T.; Crowley, T.J.; Baum, S.K.; Peltier, W.R. Neoproterozoic 'snowball Earth' simulations with a coupled climate/ice-sheet model. Nature 2000, 405, 425-429.

166. Kirschvink, J.L.; Gaidos, E.J.; Bertani, L.E.; Beukes, N.J.; Gutzmer, J.; Maepa, L.N.; Steinberger, R.E. Paleoproterozoic snowball earth: Extreme climatic and geochemical global change and its biological consequences. Proc. Natl. Acad. Sci. USA 2000, 97, 1400-1405.

167. Sessions, A.L.; Doughty, D.M.; Welander, P.V.; Summons, R.E.; Newman, D.K. The continuing puzzle of the great oxidation event. Curr. Biol. 2009, 19, R567-R574.

168. Kleidon, A. Life, hierarchy, and the thermodynamic machinery of planet Earth. Phys. Life Rev. 2010, 7, 424-460.

169. Brown, G.C.; Mussett, A.E. The Inaccessible Earth: An Integrated View to Its Structue and Composition, 2nd ed.; Chapman \& Hall: London; New York, NY, USA, 1993; p. 276.

170. Alfe, D.; Price, G.D.; Gillan, M.J. Oxygen in the Earth's core: A first-principles study. Phys. Earth Planet. Inter. 1999, 110, 191-210.

171. Dai, W.; Song, X.D. Detection of motion and heterogeneity in Earth's liquid outer core. Geophys. Res. Lett. 2008, doi:10.1029/2008GL034895 .

172. Lutgens, F.K.; Tarbuck, E.J. Essentials of Geology, 6th ed.; Prentice Hall: Upper Saddle River, NJ, USA, 1998; p. 450.

173. Jacoby, W.R. Successes and failures in geodynamics: From past to future. J. Geodyn. 2001, 32, 3-27.

174. Jordan, P. The Expanding Earth; Some Consequences of Dirac's Gravitation Hypothesis, 1st ed.; Pergamon Press: Oxford, New York, NY, USA, 1971; p. 202.

175. Betelev, N.P. The concept of an expanding earth. J. Volcanol. Seismol. 2009, 3, 355-362.

176. Van Kranendonk, M.J. Volcanic degassing, hydrothermal circulation and the flourishing of early life on Earth: A review of the evidence from c. 3490-3240 Ma rocks of the Pilbara Supergroup, Pilbara Craton, Western Australia. Earth Sci. Rev. 2006, 74, 197-240.

177. Smith, A.D.; Lewis, C. The planet beyond the plume hypothesis. Earth Sci. Rev. 1999, 48, 135-182.

178. Rogers, J.J.W.; Santosh, M. Supercontinents in earth history. Gondwana Res. 2003, 6, 357-368.

179. Dewit, M.J.; Hart, R.A. Earths earliest continental lithosphere, hydrothermal flux and crustal recycling. Lithos 1993, 30, 309-335.

180. de Wit, M.J. On Archean granites, greenstones, cratons and tectonics: Does the evidence demand a verdict? Precambrian Res. 1998, 91, 181-226.

181. Perez-Malvaez, C.; Alfredo, B.H.; Manuel, F.O.; Rosaura, R.R. Ninety-four years of the theory of the continental drift of Alfred Lothar Wegener. Interciencia 2006, 31, 536-543.

182. Varga, P. On origins of geodynamics and of modern seismology. Acta Geod. Geophys. Hungar. 2010, 45, 231-252.

183. Ito, K. Towards a new view of earthquake phenomena. Pure Appl. Geophys. 1992, 138, 531-548.

184. Freund, F. Pre-earthquake signals: Underlying physical processes. J. Asian Earth Sci. 2011, 41, 383-400.

185. Teisseyre, R.; Takeo, M.; Majewski, E. Earthquake Source Asymmetry, Structural Media and Rotation Effects. Springer: Berlin, Germany and New York, NY, USA, 2006; p. 582. 
186. Harris, R.A. Earthquake stress triggers, stress shadows, and seismic hazard. Curr. Sci. 2000, 79, 1215-1225.

187. Johnston, A.C.; Schweig, E.S. The enigma of the New Madrid earthquakes of 1811-1812. Annu. Rev. Earth Planet. Sci. 1996, 24, 339-384.

188. Simpson, J.F. Solar activity as a triggering mechanism for earthquakes. Earth Planet. Sci. Lett. 1967, 3, 417-425.

189. Canup, R.M.; Asphaug, E. Origin of the Moon in a giant impact near the end of the Earth's formation. Nature 2001, 412, 708-712.

190. Taylor, S.R. Origin of the terrestrial planets and the moon. J. R. Soc. West. Aust. 1996, 79 Pt 1, 59-65.

191. Singer, S.F.; Bandermann, L.W. Where was the moon formed? Science 1970, 170, 438-439.

192. Hartmann, W.K.; Phillips, R.J.; Taylor, G.J. Origin of the Moon; Lunar \& Planetary Institute: Houston, TX, USA, 1986; p. 781.

193. Hughes, D.W. The open question in selenology. Nature 1987, 327, doi:10.1038/327291a0.

194. Prettyman, T.H.; Hagerty, J.J.; Elphic, R.C.; Feldman, W.C.; Lawrence, D.J.; McKinney, G.W.; Vaniman, D.T. Elemental composition of the lunar surface: Analysis of gamma ray spectroscopy data from Lunar Prospector. J. Geophys. Res. Planets 2006, 111, doi:10.1029/2005JE002656.

195. Kleine, T.; Palme, H.; Mezger, K.; Halliday, A.N. Hf-W chronometry of lunar metals and the age and early differentiation of the Moon. Science 2005, 310, 1671-1674.

196. In addition to resembling the superceded fission hypothesis, this theoretical relationship is spot-on with calculations that time Earth's origin at 4.54 billion years ago (bya) and the Moon's origin at $\sim 4.52$ bya, within $\sim 50$ million years of Solar System origin.

197. Hauri, E.H.; Weinreich, T.; Saal, A.E.; Rutherford, M.C.; van Orman, J.A. High pre-eruptive water contents preserved in lunar melt inclusions. Science 2011, 333, 213-215.

198. Clark, R.N. Detection of adsorbed water and hydroxyl on the Moon. Science 2009, 326, 562-564.

199. Pieters, C.M.; Goswami, J.N.; Clark, R.N.; Annadurai, M.; Boardman, J.; Buratti, B.; Combe, J.P.; Dyar, M.D.; Green, R.; Head, J.W.; et al. Character and spatial distribution of $\mathrm{OH} / \mathrm{H} 2 \mathrm{O}$ on the surface of the Moon seen by M3 on Chandrayaan-1. Science 2009, 326, 568-572.

200. Ward, W.R. Past orientation of the lunar spin axis. Science 1975, 189, 377-379.

201. Foster, R.G.; Roenneberg, T. Human responses to the geophysical daily, annual and lunar cycles. Curr. Biol. 2008, 18, R784-R794.

202. Keeling, C.D.; Whorf, T.P. The 1800-year oceanic tidal cycle: A possible cause of rapid climate change. Proc. Natl. Acad. Sci. USA 2000, 97, 3814-3819.

203. Trask, N.J.; Rowan, L.C. Lunar Orbiter Photographs: Some Fundamental Observations: Preliminary study reveals details of craters, crater distributions, and the major types of terrain. Science 1967, 158, 1529-1535.

204. Alvarez, W.; Claeys, P.; Kieffer, S.W. Emplacement of cretaceous-tertiary boundary shocked quartz from chicxulub crater. Science 1995, 269, 930-935.

205. Zahnle, K.; Dones, L.; Levison, H.F. Cratering rates on the Galilean satellites. Icarus 1998, 136, 202-222.

206. In other words, this theory indicates that craters are not due to impact (from without to within) but rather from "expact" (a cratering force exerted from within to without). 
207. Catling, D.C.; Glein, C.R.; Zahnle, K.J.; Mckay, C.P. Why O-2 is required by complex life on habitable planets and the concept of planetary "oxygenation time". Astrobiology 2005, 5, 415-438.

208. Farquhar, J.; Johnston, D.T. The oxygen cycle of the terrestrial planets: Insights into the processing and history of oxygen in surface environments. Rev. Mineral. Geochem. 2008, 68, 463-492.

209. De Leeuw, N.H.; Catlow, C.R.; King, H.E.; Putnis, A.; Muralidharan, K.; Deymier, P.; Stimpfl, M.; Drake, M.J. Where on Earth has our water come from? Chem. Commun. 2010, 46, 8923-8925.

210. Valencia, D.; Sasselov, D.D.; O’Connell, R.J. Radius and structure models of the first superearth planet. Astrophys. J. 2007, 656, 545-551.

211. Segura, A.; Walkowicz, L.M.; Meadows, V.; Kasting, J.; Hawley, S. The effect of a strong stellar flare on the atmospheric chemistry of an earth-like planet orbiting an M dwarf. Astrobiology 2010, 10, 751-771.

212. Hegstrom, R.A.; Chamberlain, J.P.; Seto, K.; Watson, R.G. Mapping the weak chirality of atoms. Am.J. Phys. 1988, 56, 1086-1092.

213. Bai, J.; Wang, J.; Zeng, X.C. Multiwalled ice helixes and ice nanotubes. Proc. Natl. Acad. Sci. USA 2006, 103, 19664-19667.

214. Perez, S.; Bertoft, E. The molecular structures of starch components and their contribution to the architecture of starch granules: A comprehensive review. Starch Starke 2010, 62, 389-420.

215. Wright, A.J.; Jackson, L.E.; Kariuki, B.M.; Smith, M.E.; Barralet, J.E. Synthesis and structure of a calcium polyphosphate with a unique criss-cross arrangement of helical phosphate chains. Chem. Mater. 2005, 17, 4642-4646.

216. Korostelev, A.; Trakhanov, S.; Laurberg, M.; Noller, H.F. Crystal structure of a $70 \mathrm{~S}$ ribosome-tRNA complex reveals functional interactions and rearrangements. Cell 2006, 126, 1065-1077.

217. Liu, Q.; Greimann, J.C.; Lima, C.D. Reconstitution, activities, and structure of the eukaryotic RNA exosome. Cell 2006, 127, 1223-1237.

218. Luger, K.; Mader, A.W.; Richmond, R.K.; Sargent, D.F.; Richmond, T.J. Crystal structure of the nucleosome core particle at 2.8 A resolution. Nature 1997, 389, 251-260.

219. Cairns-Smith, A.G. Seven Clues to the Origin of Life: A Scientific Detective Story; Cambridge University Press: Cambridge, UK and New York, NY, USA, 1990; p. 131.

220. Calusaru, A. Why life can be only a carbon based chemistry. Rev. Roum. Chim. 1989, 34, 1787-1798.

221. Levine, J.S.; Augustsson, T.R.; Natarajan, M. The prebiological paleoatmosphere: Stability and composition. Orig. Life 1982, 12, 245-259.

222. Kasting, J.F. The evolution of the prebiotic atmosphere. Orig. Life 1984, 14, 75-82.

223. Chittenden, G.J.; Schwartz, A.W. Prebiotic photosynthetic reactions. Biol. Syst. 1981, 14, 15-32.

224. Osborne, C.P.; Beerling, D.J. Nature's green revolution: The remarkable evolutionary rise of C4 plants. Philos. Trans. R. Soc. Lond. Ser. B 2006, 361, 173-194.

225. Liedl, K.R.; Hage, W.; Hallbrucker, A.; Mayer, E. Carbonic acid in the gas phase and its astrophysical relevance. Science 1998, 279, 1332-1335. 
226. Garg, L.C.; Maren, T.H. The rates of hydration of carbon dioxide and dehydration of carbonic acid at 37 degrees. Biochim. Biophys. Acta 1972, 261, 70-76.

227. Harris, D.C. Charles David Keeling and the story of atmospheric $\mathrm{CO}_{2}$ measurements. Anal. Chem. 2010, 82, 7865-7870.

228. Davis, S.J.; Caldeira, K.; Matthews, H.D. Future $\mathrm{CO}_{2}$ emissions and climate change from existing energy infrastructure. Science 2010, 329, 1330-1333.

229. Adams, J.M.; Piovesan, G. Long series relationships between global interannual $\mathrm{CO}_{2}$ increment and climate: Evidence for stability and change in role of the tropical and boreal-temperate zones. Chemosphere 2005, 59, 1595-1612.

230. Schubert, E. The theory of and experimentation into respiratory gas exchange - Carl Ludwig and his school. Pflugers Arch. 1996, 432, R111-119.

231. Piiper, J. Carbon dioxide-oxygen relationships in gas exchange of animals. In memory of Hermann Rahn. Boll. Soc. Ital. Biol. Sper. 1991, 67, 635-658.

232. Casey, J.R. Why bicarbonate? Biochem. Cell Biol. 2006, 84, 930-939.

233. Dore, J.E.; Lukas, R.; Sadler, D.W.; Church, M.J.; Karl, D.M. Physical and biogeochemical modulation of ocean acidification in the central North Pacific. Proc. Natl. Acad. Sci. USA 2009, 106, 12235-12240.

234. Flores, C.L.; Rodriguez, C.; Petit, T.; Gancedo, C. Carbohydrate and energy-yielding metabolism in non-conventional yeasts. FEMS Microbiol. Rev. 2000, 24, 507-529.

235. Siebers, B.; Schonheit, P. Unusual pathways and enzymes of central carbohydrate metabolism in Archaea. Curr. Opin. Microbiol. 2005, 8, 695-705.

236. Kandler, O.; Gibbs, M. Asymmetric distribution of $\mathrm{C}$ in the glucose phosphates formed during photosynthesis. Plant Physiology 1956, 31, 411-412.

237. Fraser, N.J.; Hashimoto, H.; Cogdell, R.J. Carotenoids and bacterial photosynthesis: The story so far. Photosynth. Res. 2001, 70, 249-256.

238. Cleaves, H.J. The prebiotic geochemistry of formaldehyde. Precambrian Res. 2008, 164, 111-118.

239. Kalapos, M.P. A possible evolutionary role of formaldehyde. Exp. Mol. Med. 1999, 31, 1-4.

240. Feng, S.H.; Tian, G.; He, C.; Yuan, H.M.; Mu, Y.; Wang, Y.W.; Wang, L. Hydrothermal biochemistry: From formaldehyde to oligopeptides. J. Mater. Sci. 2008, 43, 2418-2425.

241. Sutherland, J.D.; Weaver, G.W. Synthesis of bis(glycoaldehyde) phosphodiester and mixed glycoaldehyde-triose phosphodiesters. Tetrahedron Lett. 1994, 35, 9109-9112.

242. Toxvaerd, S. Homochirality in bio-organic systems and glyceraldehyde in the formose reaction. J. Biol. Phys. 2005, 31, 599-606.

243. Hazen, R.M.; Deamer, D.W. Hydrothermal reactions of pyruvic acid: Synthesis, selection, and self-assembly of amphiphilic molecules. Orig. Life Evol. Biosph. 2007, 37, 143-152.

244. Martin, S.T.; Guzman, M.I. Prebiotic metabolism: Production by mineral photoelectrochemistry of alpha-ketocarboxylic acids in the reductive tricarboxylic acid cycle. Astrobiology 2009, 9, 833-842.

245. Lazcano, A.; Dworkin, J.P.; Miller, S.L. The roads to and from the RNA world. J. Theor. Biol. 2003, 222, 127-134.

246. Bielski, R.; Tencer, M. A possible path to the RNA world: Enantioselective and diastereoselective purification of ribose. Orig. Life Evol. Biosph. 2007, 37, 167-175. 
247. Fiechter, A.; Fuhrmann, G.F.; Kappeli, O. Regulation of glucose metabolism in growing yeast cells. Adv. Microb. Physiol. 1981, 22, 123-183.

248. Fukasawa, T.; Nogi, Y. Molecular genetics of galactose metabolism in yeast. Biotechnology 1989, 13, 1-18.

249. Benner, S.A.; Kim, H.J.; Ricardo, A.; Illangkoon, H.I.; Kim, M.J.; Carrigan, M.A.; Frye, F. Synthesis of carbohydrates in mineral-guided prebiotic cycles. J. Am. Chem. Soc. 2011, 133, 9457-9468.

250. Berner, R.A. The long-term carbon cycle, fossil fuels and atmospheric composition. Nature 2003, 426, 323-326.

251. Kasting, J.F.; Siefert, J.L. Life and the evolution of Earth's atmosphere. Science 2002, 296, 1066-1068.

252. Nunn, J.F. Evolution of the atmosphere. Proc. Geol. Assoc. 1998, 109, 1-13.

253. Hohmann-Marriott, M.F.; Blankenship, R.E. Evolution of photosynthesis. Annu. Rev. Plant Biol. 2011, 62, 515-548.

254. Xiong, J.; Fischer, W.M.; Inoue, K.; Nakahara, M.; Bauer, C.E. Molecular evidence for the early evolution of photosynthesis. Science 2000, 289, 1724-1730.

255. Heinrich, R.; Melendez-Hevia, E.; Montero, F.; Nuno, J.C.; Stephani, A.; Waddell, T.G. The structural design of glycolysis: An evolutionary approach. Biochem. Soc. Trans. 1999, 27, 294-298.

256. Plaxton, W.C. The Organization and Regulation of Plant Glycolysis. Annu. Rev. Plant Physiol. Plant Mol. Biol. 1996, 47, 185-214.

257. Guest, J.R.; Russell, G.C. Complexes and complexities of the citric acid cycle in Escherichia coli. Curr. Top. Cell. Regul. 1992, 33, 231-247.

258. Thauer, R.K. Citric-acid cycle, 50 years on. Modifications and an alternative pathway in anaerobic bacteria. Eur. J. Biochem. FEBS 1988, 176, 497-508.

259. Martin, W.; Schnarrenberger, C. The evolution of the Calvin cycle from prokaryotic to eukaryotic chromosomes: A case study of functional redundancy in ancient pathways through endosymbiosis. Curr. Genet. 1997, 32, 1-18.

260. Orgel, L.E. Self-organizing biochemical cycles. Proc. Natl. Acad. Sci. USA 2000, 97, 12503-12507.

261. Holland, H.D. The Chemistry of the Atmosphere and Oceans; Wiley: New York, NY, USA, 1978; p. 351.

262. Cintas, P. Tracing the origins and evolution of chirality and handedness in chemical language. Angew. Chem. 2007, 46, 4016-4024.

263. Ribo, J.M.; Crusats, J.; Sagues, F.; Claret, J.; Rubires, R. Chiral sign induction by vortices during the formation of mesophases in stirred solutions. Science 2001, 292, 2063-2066.

264. Imberty, A.; Chanzy, H.; Perez, S.; Buleon, A.; Tran, V. The double-helical nature of the crystalline part of A-starch. J. Mol. Biol. 1988, 201, 365-378.

265. Vietor, R.J.; Newman, R.H.; Ha, M.A.; Apperley, D.C.; Jarvis, M.C. Conformational features of crystal-surface cellulose from higher plants. Plant J. 2002, 30, 721-731.

266. Sikorski, P.; Hori, R.; Wada, M. Revisit of alpha-chitin crystal structure using high resolution X-ray diffraction data. Biomacromolecules 2009, 10, 1100-1105. 
267. Hirabayashi, J. On the origin of glycome and saccharide recognition. Trends Glycosci. Glycotechnol. 2004, 16, 63-85.

268. Head, I.M.; Jones, D.M.; Larter, S.R. Biological activity in the deep subsurface and the origin of heavy oil. Nature 2003, 426, 344-352.

269. Glasby, G.P. Abiogenic origin of hydrocarbons: An historical overview. Resour. Geol. 2006, 56, 85-98.

270. Rushdi, A.I.; Simoneit, B.R. Abiotic synthesis of organic compounds from carbon disulfide under hydrothermal conditions. Astrobiology 2005, 5, 749-769.

271. McCollom, T.M.; Simoneit, B.R. Abiotic formation of hydrocarbons and oxygenated compounds during thermal decomposition of iron oxalate. Orig. Life Evol. Biosph. 1999, 29, 167-186.

272. Sugisaki, R.; Mimura, K. Mantle hydrocarbons: Abiotic or biotic? Geochim. Cosmochim. Acta 1994, 58, 2527-2542.

273. Pavlov, A.A.; Kasting, J.F.; Brown, L.L.; Rages, K.A.; Freedman, R. Greenhouse warming by CH4 in the atmosphere of early Earth. J. Geophys. Res. Planets 2000, 105, 11981-11990.

274. Sorokhtin, O.G. Generation of abiogenic methane in the earth's history. Oceanology 2005, 45, 500-510.

275. Vyshemirskii, V.S.; Kontorovich, A.E. Evolution of the formation of gaseous hydrocarbons in the Earth's history. Geol. I Geofiz. 1998, 39, 1392-1401.

276. Matthews, C.N. Hydrogen cyanide polymerization: A preferred cosmochemical pathway. $J$. $B r$. Interplanet. Soc. 1992, 45, 43-48.

277. Colin-Garcia, M.; Negron-Mendoza, A.; Ramos-Bernal, S. Organics produced by irradiation of frozen and liquid HCN solutions: Implications for chemical evolution studies. Astrobiology 2009, 9, 279-288.

278. This includes carbides ( $[\mathrm{C}]_{1}$ models $\mathrm{C}^{4-},[\mathrm{C}]_{2}$ is $\mathrm{C}_{2}{ }^{4-}$, and $[\mathrm{C}]_{3}$ is $\mathrm{C}_{3}{ }^{2-}$; note the triquantal form) and cyanides (-CN, where $\mathrm{N}$ models the quantized e), the latter being thought especially important to the origin of essential biopolymers. Recall that the particle has quantal potential $\left(\mathrm{G}_{\mathrm{III}-1}\right)$ such that the link between the $\mathrm{C}$ atoms can be e, 2e, 3e. Modeling further, an e (electron) gyrolink between $\mathrm{C}$ gyromodules accounts for all the alkanes (linear or cyclic), the single bond between carbon atoms; the $2 \mathrm{e}$ (dielectron) gyrolink models alkenes, the double bond; and the $3 \mathrm{e}$ (trielectron) gyrolink models alkynes, the triple bond. (Again, please note the triquantal organization.) The cycling and re-organization of electrons is found in any other organic chemicals, for example, as in alkadienes.

279. Cheng, Q.; Thomas, S.M.; Rouviere, P. Biological conversion of cyclic alkanes and cyclic alcohols into dicarboxylic acids: Biochemical and molecular basis. Appl. Microbiol. Biotechnol. 2002, 58, 704-711.

280. Spormann, A.M.; Widdel, F. Metabolism of alkylbenzenes, alkanes, and other hydrocarbons in anaerobic bacteria. Biodegradation 2000, 11, 85-105.

281. MacFarland, H.N. Toxicology of petroleum hydrocarbons. Occup. Med. 1988, 3, 445-454.

282. Catling, D.C.; Zahnle, K.J.; McKay, C. Biogenic methane, hydrogen escape, and the irreversible oxidation of early Earth. Science 2001, 293, 839-843.

283. Bender, M.L.; Battle, M.; Keeling, R.F. The $\mathrm{O}_{2}$ balance of the atmosphere: A tool for studying the fate of fossil-fuel $\mathrm{CO}_{2}$. Annu. Rev. Energy Environ. 1998, 23, 207-223. 
284. Wolfson, R. Energy, Environment, and Climate, 1st ed.; W.W. Norton \& Company: New York, NY, USA, 2008; p. 532.

285. Richmond, G.L.; McFearin, C.L.; Beaman, D.K.; Moore, F.G. From Franklin to today: Toward a molecular level understanding of bonding and adsorption at the oil-water interface. $J$. Phys. Chem. C 2009, 113, 1171-1188.

286. Trevors, J.T. Possible origin of a membrane in the subsurface of the Earth. Cell Biol. Int. 2003, 27, 451-457.

287. Weber, A.L. Chemical constraints governing the origin of metabolism: The thermodynamic landscape of carbon group transformations under mild aqueous conditions. Orig. Life Evol. Biosph. 2002, 32, 333-357.

288. Further, the exchanged electron in these oxygyres can represent any primary, secondary, or tertiary electrogyre or combination thereof. Finally, because a carbyon particle exists in either one gyrostate or another in spacetime $\left(\mathrm{G}_{\mathrm{V}}\right)$, its arrangement within a polymer can undergo gyrostate interconversion depending upon the gyradaptive forces of the oxyon.

289. Monnard, P.A.; Maurer, S.E.; Deamer, D.W.; Boncella, J.M. Chemical evolution of amphiphiles: Glycerol monoacyl derivatives stabilize plausible prebiotic membranes. Astrobiology 2009, 9, 979-987.

290. Ohlrogge, J.B.; Jaworski, J.G. Regulation of Fatty Acid Synthesis. Annu. Rev. Plant Physiol. Plant Mol. Biol. 1997, 48, 109-136.

291. Slabas, A.R.; Brown, A.; Sinden, B.S.; Swinhoe, R.; Simon, J.W.; Ashton, A.R.; Whitfeld, P.R.; Elborough, K.M. Pivotal reactions in fatty acid synthesis. Prog. Lipid Res. 1994, 33, 39-46.

292. Weber, A.L. Origin of fatty-acid synthesis - Thermodynamics and kinetics of reaction pathways. J. Mol. Evol. 1991, 32, 93-100.

293. Huber, C.; Wachtershauser, G. Activated acetic acid by carbon fixation on (Fe,Ni)S under primordial conditions. Science 1997, 276, 245-247.

294. Goldanskii, V.I. Cold prebiotic evolution, tunneling, chirality and exobiology. Phys. Orig. Homochirality Life 1996, 211-230.

295. Venema, K.; Al-Lahham, S.H.; Peppelenbosch, M.P.; Roelofsen, H.; Vonk, R.J. Biological effects of propionic acid in humans; metabolism, potential applications and underlying mechanisms. Biochim. Biophys. Acta 2010, 1801, 1175-1183.

296. Beauchamp, E.; Rioux, V.; Legrand, P. New regulatory and signal functions for myristic acid. Med. Sci. 2009, 25, 57-63.

297. Dabadie, H.; Peuchant, E.; Motta, C.; Bernard, M.; Mendy, F. Myristic acid: Effects on HDL, omega3, LDL oxidation and membrane fluidity. Sci. Des Aliment. 2008, 28, 134-142.

298. Biochemistry of Lipids, Lipoproteins and Membranes, 5th ed.; Vance, D.E., Vance, J.E., Eds.; Elsevier: Amsterdam, The Netherlands and Boston, MA, USA, 2008; p. 631.

299. Kuksis, A. Fatty Acids and Glycerides; Plenum Press: New York, NY, USA, 1978; p. 469.

300. Fujita, Y.; Matsuoka, H.; Hirooka, K. Regulation of fatty acid metabolism in bacteria. Mol. Microbiol. 2007, 66, 829-839.

301. Van Roermund, C.W.; Waterham, H.R.; Ijlst, L.; Wanders, R.J. Fatty acid metabolism in Saccharomyces cerevisiae. Cell. Mol. Life Sci. 2003, 60, 1838-1851. 
302. Small, D.M. The effects of glyceride structure on absorption and metabolism. Annu. Rev. Nutr. 1991, 11, 413-434.

303. Hsieh, H.H.; Jewitt, D. A population of comets in the main asteroid belt. Science 2006, 312, 561-563.

304. Brown, M.E.; Barkume, K.M.; Ragozzine, D.; Schaller, E.L. A collisional family of icy objects in the Kuiper belt. Nature 2007, 446, 294-296.

305. Michel, P.; Benz, W.; Richardson, D.C. Disruption of fragmented parent bodies as the origin of asteroid families. Nature 2003, 421, 608-611.

306. Levison, H.F.; Duncan, M.J.; Brasser, R.; Kaufmann, D.E. Capture of the Sun's Oort cloud from stars in its birth cluster. Science 2010, 329, 187-190.

307. Stern, S.A. The evolution of comets in the Oort cloud and Kuiper belt. Nature 2003, 424, 639-642.

308. Sunshine, J.M.; A’Hearn, M.F.; Groussin, O.; Li, J.Y.; Belton, M.J.; Delamere, W.A.; Kissel, J.; Klaasen, K.P.; McFadden, L.A.; Meech, K.J.; et al. Exposed water ice deposits on the surface of comet 9P/Tempel 1. Science 2006, 311, 1453-1455.

309. Mumma, M.J.; DiSanti, M.A.; Dello Russo, N.; Fomenkova, M.; Magee-Sauer, K.; Kaminski, C.D.; Xie, D.X. Detection of abundant ethane and methane, along with carbon monoxide and water, in comet C/1996 B2 Hyakutake: Evidence for interstellar origin. Science 1996, 272, 1310-1314.

310. Kirschvink, J.L.; Maine, A.T.; Vali, H. Paleomagnetic evidence of a low-temperature origin of carbonate in the Martian meteorite ALH84001. Science 1997, 275, 1629-1633.

311. Fischer, T.P.; Burnard, P.; Marty, B.; Hilton, D.R.; Furi, E.; Palhol, F.; Sharp, Z.D.; Mangasini, F. Upper-mantle volatile chemistry at Oldoinyo Lengai volcano and the origin of carbonatites. Nature 2009, 459, 77-80.

312. Cooper, G.; Kimmich, N.; Belisle, W.; Sarinana, J.; Brabham, K.; Garrel, L. Carbonaceous meteorites as a source of sugar-related organic compounds for the early Earth. Nature 2001, 414, 879-883.

313. For example, cometary orbit around planets and their moons is modeled as an ohiogyre, with the binary planet-moon system exerting the attractorepulsive effects that adapt and sustain the cyclical path.

314. Free, A.; Barton, N.H. Do evolution and ecology need the Gaia hypothesis? Trends Ecol. Evol. 2007, 22, 611-619.

315. Kerr, R.A. No Longer Willful, Gaia Becomes Respectable: The Gaia hypothesis, that Earth is a single huge organism intentionally creating an optimum environment for itself; has been made more palatable; interesting science is coming of it. Science 1988, 240, 393-395.

316. Lovelock, J. Gaia: The Practical Science of Planetary Medicine; Oxford University Press: Oxford, UK and New York, NY, USA, 2000; p. 192.

317. Monnard, P.A.; Deamer, D.W. Membrane self-assembly processes: Steps toward the first cellular life. The Anatomical Record 2002, 268, 196-207.

318. Norris, V.; Raine, D.J. A fission-fusion origin for life. Orig. Life Evol. Biosph. 1998, 28, 523-537.

319. Maddox, J. Origin of the first cell membrane? Nature 1994, 371, doi:10.1038/371101a0. 
320. Lipmann, F. Metabolic Generation and Utilization of Phosphate Bond Energy; Wiley: Hoboken, NJ, USA, 2006; Volume 1.

321. Pasek, M.A. Rethinking early Earth phosphorus geochemistry. Proc. Natl. Acad. Sci. USA 2008, 105, 853-858.

322. Schopf, J.W.; Packer, B.M. Early Archean (3.3-billion to 3.5-billion-year-old) microfossils from Warrawoona Group, Australia. Science 1987, 237, 70-73.

323. Knoll, A.H. Life on a Young Planet: The First Three Billion Years of Evolution on Earth; Princeton University Press: Princeton, NJ, USA, 2003; p. 277.

324. Liebl, V.; Novak, V.J.; Masinovsky, Z.; Pacltova, B.; Bejsovcova, L. The evolution of prebiological self-organization: Probable colloid-chemical evolution of first prokaryotic cells. Orig. Life 1984, 14, 323-334.

325. Luisi, P.L.; Rasi, P.S.; Mavelli, F. A possible route to prebiotic vesicle reproduction. Artif. Life 2004, 10, 297-308.

326. Monnard, P.A.; Ziock, H.J. Question 9: Prospects for the construction of artificial cells or protocells. Orig. Life Evol. Biosph. 2007, 37, 469-472.

327. Qian, H.; Beard, D.A. Thermodynamics of stoichiometric biochemical networks in living systems far from equilibrium. Biophys. Chem. 2005, 114, 213-220.

328. Alberty, R.A. Biochemical thermodynamics. Biochim. Biophys. Acta 1994, 1207, 1-11.

329. Walsh, C.T.; Benson, T.E.; Kim, D.H.; Lees, W.J. The versatility of phosphoenolpyruvate and its vinyl ether products in biosynthesis. Chem. Biol. 1996, 3, 83-91.

330. Gabor, E.; Gohler, A.K.; Kosfeld, A.; Staab, A.; Kremling, A.; Jahreis, K. The phosphoenolpyruvate-dependent glucose-phosphotransferase system from Escherichia coli K-12 as the center of a network regulating carbohydrate flux in the cell. Eur. J. Cell Biol. 2011, 90, 711-720.

331. McCleary, W.R.; Stock, J.B.; Ninfa, A.J. Is acetyl phosphate a global signal in Escherichia coli? J. Bacteriol. 1993, 175, 2793-2798.

332. Wolfe, A.J.; Chang, D.E.; Walker, J.D.; Seitz-Partridge, J.E.; Vidaurri, M.D.; Lange, C.F.; Pruss, B.M.; Henk, M.C.; Larkin, J.C.; Conway, T. Evidence that acetyl phosphate functions as a global signal during biofilm development. Mol. Microbiol. 2003, 48, 977-988.

333. Hers, H.G.; Hue, L. Gluconeogenesis and related aspects of glycolysis. Annu. Rev. Biochem. 1983, 52, 617-653.

334. Mather, M.W.; Gennis, R.B. Kinetic studies of the lipid-activated pyruvate oxidase flavoprotein of Escherichia coli. J. Biol. Chem. 1985, 260, 16148-16155.

335. Svensson, P.; Blasing, O.E.; Westhoff, P. Evolution of C4 phosphoenolpyruvate carboxylase. Arch. Biochem. Biophys. 2003, 414, 180-188.

336. Comte, B.; Vincent, G.; Bouchard, B.; Des Rosiers, C. Probing the origin of acetyl-CoA and oxaloacetate entering the citric acid cycle from the $13 \mathrm{C}$ labeling of citrate released by perfused rat hearts. J. Biol. Chem. 1997, 272, $26117-26124$.

337. Kent, C.; Carman, G.M.; Spence, M.W.; Dowhan, W. Regulation of eukaryotic phospholipid metabolism. FASEB J. 1991, 5, 2258-2266.

338. Alb, J.G., Jr.; Kearns, M.A.; Bankaitis, V.A. Phospholipid metabolism and membrane dynamics. Curr. Opin. Cell. Biol. 1996, 8, 534-541. 
339. Hamilton, J.A. Fatty acid transport: Difficult or easy? J. Lipid Res. 1998, 39, 467-481.

340. Walter, A.; Kuehl, G.; Barnes, K.; VanderWaerdt, G. The vesicle-to-micelle transition of phosphatidylcholine vesicles induced by nonionic detergents: Effects of sodium chloride, sucrose and urea. Biochim. Biophys. Acta 2000, 1508, 20-33.

341. Lichtenberg, D.; Opatowski, E.; Kozlov, M.M. Phase boundaries in mixtures of membrane-forming amphiphiles and micelle-forming amphiphiles. Biochim. Biophys. Acta 2000, 1508, 1-19.

342. Zhang, J.; Jing, B.; Tokutake, N.; Regen, S.L. Transbilayer complementarity of phospholipids. A look beyond the fluid mosaic model. J. Am. Chem. Soc. 2004, 126, 10856-10857.

343. Wisniewska, A.; Draus, J.; Subczynski, W.K. Is a fluid-mosaic model of biological membranes fully relevant? Studies on lipid organization in model and biological membranes. Cell. Mol. Biol. Lett. 2003, 8, 147-159.

344. Sinensky, M. Homeoviscous adaptation - a homeostatic process that regulates the viscosity of membrane lipids in Escherichia coli. Proc. Natl. Acad. Sci. USA 1974, 71, 522-525.

345. Williams, N.H. Models for biological phosphoryl transfer. Biochim. Biophys. Acta 2004, 1697, 279-287.

346. Florian, J.; Warshel, A. A fundamental assumption about $\mathrm{OH}-$ attack in phosphate ester hydrolysis is not fully justified. J. Am. Chem. Soc. 1997, 119, 5473-5474.

347. Meister, A. Carboxy phosphate: An intermediate in the enzymatic synthesis of carbamyl phosphate. Trans. N. Y. Acad. Sci. 1983, 41, 117-128.

348. Simons, K.; Ikonen, E. Functional rafts in cell membranes. Nature 1997, 387, 569-572.

349. Zhang, J.; Jing, B.; Janout, V.; Regen, S.L. Detecting cross talk between two halves of a phospholipid bilayer. Langmuir 2007, 23, 8709-8712.

350. Klute, M.J.; Melancon, P.; Dacks, J.B. Evolution and diversity of the Golgi. Cold Spring Harbor Perspect. Biol. 2011, 3, doi:10.1101/cshperspect.a007849.

351. Sparkes, I.A.; Frigerio, L.; Tolley, N.; Hawes, C. The plant endoplasmic reticulum: A cell-wide web. Biochem. J. 2009, 423, 145-155.

352. Mironov, A.A.; Banin, V.V.; Sesorova, I.S.; Dolgikh, V.V.; Luini, A.; Beznoussenko, G.V. Evolution of the endoplasmic reticulum and the Golgi complex. Adv. Exp. Med. Biol. 2007, 607, 61-72.

353. Schrader, M.; Fahimi, H.D. The peroxisome: Still a mysterious organelle. Histochem. Cell Biol. 2008, 129, 421-440.

354. Weisman, L.S. Organelles on the move: Insights from yeast vacuole inheritance. Nat. Rev. Mol. Cell Biol. 2006, 7, 243-252.

355. Dacks, J.B.; Field, M.C. Evolution of the eukaryotic membrane-trafficking system: Origin, tempo and mode. J. Cell Sci. 2007, 120, 2977-2985.

356. Nota bene: While these endomembrane systems are theoretically positioned here, the modeling the origin of these systems is premature-evolutionarily consistent modeling requires thermodynamic feedback or shunting of IEM from supervenient gyrosystems (3.5-3.8).

357. Homan, R.; Pownall, H.J. Transbilayer diffusion of phospholipids: Dependence on headgroup structure and acyl chain length. Biochim. Biophys. Acta 1988, 938, 155-166. 
358. Kamp, F.; Zakim, D.; Zhang, F.; Noy, N.; Hamilton, J.A. Fatty acid flip-flop in phospholipid bilayers is extremely fast. Biochemistry 1995, 34, 11928-11937.

359. Lamarche, M.G.; Wanner, B.L.; Crepin, S.; Harel, J. The phosphate regulon and bacterial virulence: A regulatory network connecting phosphate homeostasis and pathogenesis. FEMS Microbiol. Rev. 2008, 32, 461-473.

360. Berndt, T.; Kumar, R. Phosphatonins and the regulation of phosphate homeostasis. Annu. Rev. Physiol. 2007, 69, 341-359.

361. Del Popolo, M.G.; Ballone, P. Melting behavior of an idealized membrane model. J. Chem. Phys. 2008, 128, doi:10.1063/1.2804423.

362. Wassall, S.R.; Stillwell, W. Polyunsaturated fatty acid-cholesterol interactions: Domain formation in membranes. Biochim. Biophys. Acta 2009, 1788, 24-32.

363. Reinhart, M.P. Intracellular sterol trafficking. Experientia 1990, 46, 599-611.

364. Lucy, J.A.; Dingle, J.T. Fat-Soluble Vitamins and Biological Membranes. Nature 1964, 204, 156-160.

365. Navas, P.; Villalba, J.M.; de Cabo, R. The importance of plasma membrane coenzyme Q in aging and stress responses. Mitochondrion 2007, 7, S34-S40.

366. White, H.B. Coenzymes as fossils of an earlier metabolic state. J. Mol. Evol. 1976, 7, 101-104.

367. Hendrich, A.B. Flavonoid-membrane interactions: Possible consequences for biological effects of some polyphenolic compounds. Acta Pharmacol. Sin. 2006, 27, 27-40.

368. Lemaire-Ewing, S.; Desrumaux, C.; Neel, D.; Lagrost, L. Vitamin E transport, membrane incorporation and cell metabolism: Is alpha-tocopherol in lipid rafts an oar in the lifeboat? Mol. Nutr. Food Res. 2010, 54, 631-640.

369. Kulaev, I.; Kulakovskaya, T. Polyphosphate and phosphate pump. Annu. Rev. Microbiol. 2000, 54, 709-734.

370. Achbergerova, L.; Nahalka, J. Polyphosphate-An ancient energy source and active metabolic regulator. Microb. Cell Fact. 2011, 10, doi:10.1186/1475-2859-10-63.

371. Kornberg, A. Inorganic polyphosphate: A molecule of many functions. Progr. Mol. Subcell. Biol. 1999, 23, 1-18.

372. Crawford, G.E.; Earnshaw, J.C. Phase transitions in monoglyceride bilayers. A light scattering study. Biophys. J. 1986, 49, 869-889.

373. Rotering, H.; Raetz, C.R. Appearance of monoglyceride and triglyceride in the cell envelope of Escherichia coli mutants defective in diglyceride kinase. J. Biol. Chem. 1983, 258, 8068-8073.

374. Carrasco, S.; Merida, I. Diacylglycerol, when simplicity becomes complex. Trends Biochem. Sci. 2007, 32, 27-36.

375. Khandelia, H.; Duelund, L.; Pakkanen, K.I.; Ipsen, J.H. Triglyceride blisters in lipid bilayers: Implications for lipid droplet biogenesis and the mobile lipid signal in cancer cell membranes. PLoS One 2010, 5, doi:10.1371/journal.pone.0012811.

376. Olofsson, S.O.; Bostrom, P.; Andersson, L.; Rutberg, M.; Levin, M.; Perman, J.; Boren, J. Triglyceride containing lipid droplets and lipid droplet-associated proteins. Curr. Opin. Lipidol. 2008, 19, 441-447.

377. Graves, J.D.; Krebs, E.G. Protein phosphorylation and signal transduction. Pharmacol. Ther. 1999, 82, 111-121. 
378. Towler, D.A. Inorganic pyrophosphate: A paracrine regulator of vascular calcification and smooth muscle phenotype. Arterioscler. Thromb. Vasc. Biol. 2005, 25, 651-654.

379. Mansurova, S.E. Inorganic pyrophosphate in mitochondrial metabolism. Biochim. Biophys. Acta 1989, 977, 237-247.

380. Reusch, R.N. Transmembrane ion transport by polyphosphate/poly-(R)-3-hydroxybutyrate complexes. Biochem. Biokhimiia 2000, 65, 280-295.

381. Roels, J.; Verstraete, W. Biological formation of volatile phosphorus compounds. Bioresour. Technol. 2001, 79, 243-250.

382. Pasek, M.A.; Lauretta, D.S. Aqueous corrosion of phosphide minerals from iron meteorites: A highly reactive source of prebiotic phosphorus on the surface of the early Earth. Astrobiology 2005, 5, 515-535.

383. Schink, B.; Friedrich, M. Phosphite oxidation by sulphate reduction. Nature 2000, 406, doi: $10.1038 / 35017644$.

384. Based upon thermodynamic relationships of a focagyre with distal subgyrosystems $\left(\mathrm{G}_{\mathrm{XI}-1}\right)$, the tertiary phosphogyre models phosphate relationships with elements in the electrogyre, explaining the bioproduction and bioremediation of volatile phosphorous compounds such as phosphines and phosphides.

385. Paytan, A.; McLaughlin, K. The oceanic phosphorus cycle. Curr. Rev. 2007, 107, 563-576.

386. Cembella, A.D.; Antia, N.J.; Harrison, P.J. The utilization of inorganic and organic phosphorus compounds as nutrients by eukaryotic microalgae: A multidisciplinary perspective. Part 2. Crit. Rev. Microbiol. 1984, 11, 13-81.

387. Cembella, A.D.; Antia, N.J.; Harrison, P.J. The utilization of inorganic and organic phosphorous compounds as nutrients by eukaryotic microalgae: A multidisciplinary perspective: Part 1. Crit. Rev. Microbiol. 1984, 10, 317-391.

388. Cavalier-Smith, T. Membrane heredity and early chloroplast evolution. Trends Plant Sci. 2000, $5,174-182$.

389. Saraste, J.; Goud, B. Functional symmetry of endomembranes. Mol. Biol. Cell 2007, 18, $1430-1436$.

390. Morowitz, H.J.; Heinz, B.; Deamer, D.W. The chemical logic of a minimum protocell. Orig. Life Evol. Biosph. 1988, 18, 281-287.

391. Segre, D.; Ben-Eli, D.; Deamer, D.W.; Lancet, D. The lipid world. Orig. Life Evol. Biosph. 2001, 31, 119-145.

392. Kiss, D.L.; Andrulis, E.D. The exozyme model: A continuum of functionally distinct complexes. RNA 2011, 17, 1-13.

393. Thieffry, D.; Sarkar, S. Forty years under the central dogma. Trends Biochem. Sci. 1998, 23, 312-316.

394. Henikoff, S. Beyond the central dogma. Bioinformatics 2002, 18, 223-225.

395. Temin, H.M.; Mizutani, S. RNA-dependent DNA polymerase in virions of Rous sarcoma virus. Nature 1970, 226, 1211-1213.

396. Baltimore, D. RNA-dependent DNA polymerase in virions of RNA tumour viruses. Nature 1970, 226, 1209-1211.

397. Gilbert, W. Origin of life-The rna world. Nature 1986, 319, 618-618. 
398. Bartel, D.P.; Unrau, P.J. RNA-catalysed nucleotide synthesis. Nature 1998, 395, 260-263.

399. Eschenmoser, A. Chemical etiology of nucleic acid structure. Science 1999, 284, 2118-2124.

400. Orgel, L.E. The origin of life-A review of facts and speculations. Trends Biochem. Sci. 1998, 23, 491-495.

401. Ferris, J. Origins of life. Chemical replication. Nature 1994, 369, 184-185.

402. Ridley, A.J. Rho family proteins: Coordinating cell responses. Trends Cell Biol. 2001, 11, 471-477.

403. Prodromou, C.; Roe, S.M.; O’Brien, R.; Ladbury, J.E.; Piper, P.W.; Pearl, L.H. Identification and structural characterization of the ATP/ADP-binding site in the Hsp90 molecular chaperone. Cell 1997, 90, 65-75.

404. Chavrier, P.; Goud, B. The role of ARF and Rab GTPases in membrane transport. Curr. Opin. Cell. Biol. 1999, 11, 466-475.

405. Avis, J.M.; Clarke, P.R. Ran, a GTPase involved in nuclear processes: Its regulators and effectors. J. Cell Sci. 1996, 109, 2423-2427.

406. Anderson, C.M.; Parkinson, F.E. Potential signalling roles for UTP and UDP: Sources, regulation and release of uracil nucleotides. Trends Pharmacol. Sci. 1997, 18, 387-392.

407. Renner, A.B.; Rieger, K.; Grunow, D.; Zimmermann-Kordmann, M.; Gohlke, M.; Reutter, W. Liver-specific increase of UTP and UDP-sugar concentrations in rats induced by dietary vitamin B6-deficiency and its relation to complex N-glycan structures of liver membrane-proteins. Glycoconj. J. 2007, 24, 531-541.

408. Chang, Y.F.; Carman, G.M. CTP synthetase and its role in phospholipid synthesis in the yeast Saccharomyces cerevisiae. Prog. Lipid Res. 2008, 47, 333-339.

409. Turnock, D.C.; Ferguson, M.A. Sugar nucleotide pools of Trypanosoma brucei, Trypanosoma cruzi, and Leishmania major. Eukaryot. Cell 2007, 6, 1450-1463.

410. Mitchell, P.; Moyle, J. Chemiosmotic hypothesis of oxidative phosphorylation. Nature 1967, 213, 137-139.

411. Urata, H.; Shimizu, H.; Akagi, M. Structural studies of heterochiral DNA/DNA, RNA/RNA, AND DNA/RNA duplexes. Nucleosides Nucleotides Nucleic Acids 2006, 25, 359-367.

412. Kulaev, I.S.; Mansurova, S.E.; Burlakova, E.B.; Dukhovich, V.F. Why ATP instead of pyrophosphate-interrelation between ATP and pyrophosphate production during evolution and in contemporary organisms. Biosystems 1980, 12, 177-180.

413. This spatiotemporal and thermodynamic relationship explains the biomembrane-dependent transition from phosphorylation with pyrophosphate formation to phosphorylation with ATP formation.

414. Fuda, N.J.; Ardehali, M.B.; Lis, J.T. Defining mechanisms that regulate RNA polymerase II transcription in vivo. Nature 2009, 461, 186-192.

415. Malik, S.; Roeder, R.G. Dynamic regulation of pol II transcription by the mammalian Mediator complex. Trends Biochem. Sci. 2005, 30, 256-263.

416. Tjian, R. The biochemistry of transcription in eukaryotes: A paradigm for multisubunit regulatory complexes. Philos. Trans. R. Soc. Lond. Ser. B 1996, 351, 491-499.

417. Zamore, P.D.; Tuschl, T.; Sharp, P.A.; Bartel, D.P. RNAi: Double-stranded RNA directs the ATP-dependent cleavage of mRNA at 21 to 23 nucleotide intervals. Cell 2000, 101, 25-33. 
418. Liu, Q.; Paroo, Z. Biochemical principles of small RNA pathways. Annu. Rev. Biochem. 2010, 79, 295-319.

419. Pullirsch, D.; Jantsch, M.F. Proteome diversification by adenosine to inosine RNA editing. $R N A$ Biol. 2010, 7, 205-212.

420. Gott, J.M. Expanding genome capacity via RNA editing. Comptes Rendus Biol. 2003, 326, 901-908.

421. Roy, S.W.; Gilbert, W. The evolution of spliceosomal introns: Patterns, puzzles and progress. Nat. Rev. Genet. 2006, 7, 211-221.

422. Borek, E.; Baliga, B.S.; Gehrke, C.W.; Kuo, C.W.; Belman, S.; Troll, W.; Waalkes, T.P. High turnover rate of transfer RNA in tumor tissue. Cancer Res. 1977, 37, 3362-3366.

423. Gill, S.C.; Yager, T.D.; von Hippel, P.H. Thermodynamic analysis of the transcription cycle in E. coli. Biophys. Chem. 1990, 37, 239-250.

424. Spellman, P.T.; Sherlock, G.; Zhang, M.Q.; Iyer, V.R.; Anders, K.; Eisen, M.B.; Brown, P.O.; Botstein, D.; Futcher, B. Comprehensive identification of cell cycle-regulated genes of the yeast Saccharomyces cerevisiae by microarray hybridization. Mol. Biol. Cell 1998, 9, 3273-3297.

425. Krejcova, R.; Horska, K. Nucleoside diphosphate kinases. Chem. Listy 1997, 91, 466-476.

426. Briggs, A.G.; Bent, A.F. Poly(ADP-ribosyl)ation in plants. Trends Plant Sci. 2011, 16, 372-380.

427. Proudfoot, N. Connecting transcription to messenger RNA processing. Trends Biochem. Sci. 2000, 25, 290-293.

428. Bentley, D. Coupling RNA polymerase II transcription with pre-mRNA processing. Curr. Opin. Cell Biol. 1999, 11, 347-351.

429. McClain, W.H. Transfer RNA identity. FASEB J. 1993, 7, 72-78.

430. Sun, F.J.; Caetano-Anolles, G. Transfer RNA and the origins of diversified life. Science Progress 2008, 91, 265-284.

431. Nazar, R.N. Ribosomal RNA processing and ribosome biogenesis in eukaryotes. IUBMB Life 2004, 56, 457-465.

432. Moss, T. At the crossroads of growth control; making ribosomal RNA. Curr. Opin. Genet. Dev. 2004, 14, 210-217.

433. Barraud, P.; Schmitt, E.; Mechulam, Y.; Dardel, F.; Tisne, C. A unique conformation of the anticodon stem-loop is associated with the capacity of tRNAfMet to initiate protein synthesis. Nucleic Acids Res. 2008, 36, 4894-4901.

434. Rupert, P.B.; Ferre-D'Amare, A.R. Crystal structure of a hairpin ribozyme-inhibitor complex with implications for catalysis. Nature 2001, 410, 780-786.

435. Stahley, M.R.; Strobel, S.A. Structural evidence for a two-metal-ion mechanism of group I intron splicing. Science 2005, 309, 1587-1590.

436. Selmer, M.; Dunham, C.M.; Murphy, F.V., 4th; Weixlbaumer, A.; Petry, S.; Kelley, A.C.; Weir, J.R.; Ramakrishnan, V. Structure of the 70S ribosome complexed with mRNA and tRNA. Science 2006, 313, 1935-1942.

437. Antoni, F.A. Molecular diversity of cyclic AMP signalling. Front. Neuroendocrinol. 2000, 21, 103-132.

438. Bolwell, G.P. Cyclic AMP, the reluctant messenger in plants. Trends Biochem. Sci. 1995, 20, 492-495. 
439. Roelofs, J.; Smith, J.L.; Van Haastert, P.J. cGMP signalling: Different ways to create a pathway. Trends Genet. 2003, 19, 132-134.

440. Ryan, R.P.; Fouhy, Y.; Lucey, J.F.; Jiang, B.L.; He, Y.Q.; Feng, J.X.; Tang, J.L.; Dow, J.M. Cyclic di-GMP signalling in the virulence and environmental adaptation of Xanthomonas campestris. Mol. Microbiol. 2007, 63, 429-442.

441. Mills, E.; Pultz, I.S.; Kulasekara, H.D.; Miller, S.I. The bacterial second messenger c-di-GMP: Mechanisms of signalling. Cell. Microbiol. 2011, 13, 1122-1129.

442. Belenky, P.; Bogan, K.L.; Brenner, C. NAD+ metabolism in health and disease. Trends Biochem. Sci. 2007, 32, 12-19.

443. Muller, F. The flavin redox-system and its biological function. Topics Curr. Chem. 1983, 108, 71-107.

444. Given the emergence of vitamins in the carbogyre, and the relativistically strong creatodestructive force it exerts through the phosphogyre on the ribogyre $\left(\mathrm{G}_{\mathrm{XIII}}\right)$, the compounds flavin adenine dinucleotide and flavin mononucleotide are also positioned in the gyrobase of the secondary majorgyre.

445. Koonin, E.V.; Novozhilov, A.S. Origin and evolution of the genetic code: The universal enigma. IUBMB Life 2009, 61, 99-111.

446. Westover, K.D.; Bushnell, D.A.; Kornberg, R.D. Structural basis of transcription: Nucleotide selection by rotation in the RNA polymerase II active center. Cell 2004, 119, 481-489.

447. Anand, V.S.; Patel, S.S. Transient state kinetics of transcription elongation by T7 RNA polymerase. J. Biol. Chem. 2006, 281, 35677-35685.

448. Alvager, T.; Graham, G.; Hilleke, R.; Hutchison, D.; Westgard, J. On the information content of the genetic code. Biol. Syst. 1989, 22, 189-196.

449. Crick, F.H. Codon - anticodon pairing: The wobble hypothesis. J. Mol. Biol. 1966, 19, 548-555.

450. Forterre, P. Defining life: The virus viewpoint. Orig. Life Evol. Biosph. 2010, 40, 151-160.

451. Villarreal, L.P. Viruses and the Evolution of Life; ASM Press: Washington, DC, USA, 2005; p. 395.

452. Dadalti, P.; Goodheart, C. Did the first virus self-assemble from self-replicating prion proteins and RNA? Med. Hypotheses 2007, 69, 724-730.

453. Becker, Y. Molecular evolution of viruses: An interim summary. Virus Genes 1995, 11, 299-302.

454. Holmes, E.C. On the origin and evolution of the human immunodeficiency virus (HIV). Biol. Rev. Camb. Philos. Soc. 2001, 76, 239-254.

455. Zandi, R.; van der Schoot, P. Size regulation of ss-RNA viruses. Biophys. J. 2009, 96, 9-20.

456. Basile, B.; Lazcano, A.; Oro, J. Prebiotic syntheses of purines and pyrimidines. Adv. Space Res. 1984, 4, 125-131.

457. Sutherland, J.D. Ribonucleotides. Cold Spring Harbor Perspect. Biol. 2010, 2, a005439.

458. Tan, D.X.; Manchester, L.C.; Terron, M.P.; Flores, L.J.; Reiter, R.J. One molecule, many derivatives: A never-ending interaction of melatonin with reactive oxygen and nitrogen species? J. Pineal Res. 2007, 42, 28-42.

459. Cassone, V.M.; Natesan, A.K. Time and time again: The phylogeny of melatonin as a transducer of biological time. J. Biol. Rhythm. 1997, 12, 489-497. 
460. Stefano, G.B.; Kream, R.M. Endogenous morphine synthetic pathway preceded and gave rise to catecholamine synthesis in evolution (Review). Int. J. Mol. Med. 2007, 20, 837-841.

461. Deiters, A.; Martin, S.F. Synthesis of oxygen- and nitrogen-containing heterocycles by ring-closing metathesis. Curr. Rev. 2004, 104, 2199-2238.

462. Sahr, T.; Ravanel, S.; Rebeille, F. Tetrahydrofolate biosynthesis and distribution in higher plants. Biochem. Soc. Trans. 2005, 33, 758-762.

463. Vasileuskaya, Z.; Oster, U.; Beck, C.F. Involvement of tetrapyrroles in inter-organellar signaling in plants and algae. Photosynth. Res. 2004, 82, 289-299.

464. Kulikowska, E.; Kierdaszuk, B.; Shugar, D. Xanthine, xanthosine and its nucleotides: Solution structures of neutral and ionic forms, and relevance to substrate properties in various enzyme systems and metabolic pathways. Acta Biochim. Pol. 2004, 51, 493-531.

465. Lucock, M.; Yates, Z. Folic acid - vitamin and panacea or genetic time bomb? Nat. Rev. Genet. 2005, 6, 235-240.

466. Alvarez-Lario, B.; Macarron-Vicente, J. Uric acid and evolution. Rheumatology 2010, 49, 2010-2015.

467. Azmitia, E.C. Modern views on an ancient chemical: Serotonin effects on cell proliferation, maturation, and apoptosis. Brain Res. Bull. 2001, 56, 413-424.

468. Cuvillier, O. Sphingosine in apoptosis signaling. Biochim. Biophys. Acta 2002, 1585, 153-162.

469. Miller, S.L. Production of amino acids under possible primitive earth conditions. Science 1953, 117, 528-529.

470. Denis, V.; Daignan-Fornier, B. Synthesis of glutamine, glycine and 10-formyl tetrahydrofolate is coregulated with purine biosynthesis in Saccharomyces cerevisiae. Mol. Gen. Genet. 1998, 259, 246-255.

471. Boza, J.J.; Moennoz, D.; Bournot, C.E.; Blum, S.; Zbinden, I.; Finot, P.A.; Ballevre, O. Role of glutamine on the de novo purine nucleotide synthesis in Caco-2 cells. Eur. J. Nutr. 2000, 39, $38-46$.

472. Huang, M.; Graves, L.M. De novo synthesis of pyrimidine nucleotides; emerging interfaces with signal transduction pathways. Cell. Mol. Life Sci. 2003, 60, 321-336.

473. Traut, T.W.; Jones, M.E. Uracil metabolism-UMP synthesis from orotic acid or uridine and conversion of uracil to beta-alanine: Enzymes and cDNAs. Prog. Nucleic Acid Res. Mol. Biol. 1996, 53, 1-78.

474. Stepansky, A.; Leustek, T. Histidine biosynthesis in plants. Amino Acids 2006, 30, 127-142.

475. Gruber, N.; Galloway, J.N. An Earth-system perspective of the global nitrogen cycle. Nature 2008, 451, 293-296.

476. Navarro-Gonzalez, R.; McKay, C.P.; Mvondo, D.N. A possible nitrogen crisis for Archaean life due to reduced nitrogen fixation by lightning. Nature 2001, 412, 61-64.

477. Hamilton, I.R.; Burris, R.H.; Wilson, P.W.; Wang, C.H. Pyruvate metabolism, carbon dioxide assimilation, and nitrogen fixation by an achromobacter species. J. Bacteriol. 1965, 89, 647-653.

478. Sobko, T.; Reinders, C.I.; Jansson, E.; Norin, E.; Midtvedt, T.; Lundberg, J.O. Gastrointestinal bacteria generate nitric oxide from nitrate and nitrite. Nitric Oxide 2005, 13, 272-278.

479. Lundberg, J.O.; Weitzberg, E.; Gladwin, M.T. The nitrate-nitrite-nitric oxide pathway in physiology and therapeutics. Nat. Rev. Drug Discov. 2008, 7, 156-167. 
480. Stuehr, D.J. Enzymes of the L-arginine to nitric oxide pathway. J. Nutr. 2004, 134, 2748S-2751S; discussion 2765S-2767S.

481. Nyffeler, P.T.; Liang, C.H.; Koeller, K.M.; Wong, C.H. The chemistry of amine-azide interconversion: Catalytic diazotransfer and regioselective azide reduction. J. Am. Chem. Soc. 2002, 124, 10773-10778.

482. Brandes, J.A.; Boctor, N.Z.; Cody, G.D.; Cooper, B.A.; Hazen, R.M.; Yoder, H.S., Jr. Abiotic nitrogen reduction on the early Earth. Nature 1998, 395, 365-367.

483. On this matter, the attractorepulsive effects of the primary electrogyre on the nitrogyre models ammonia, consistent with ideas regarding its formation on the early Earth.

484. Doherty, E.A.; Doudna, J.A. Ribozyme structures and mechanisms. Annu. Rev. Biophys. Biomol. Struct. 2001, 30, 457-475.

485. Lahav, N. Prebiotic co-evolution of self-replication and translation or RNA world? J. Theor. Biol. 1991, 151, 531-539.

486. Lilley, D.M. The ribosome functions as a ribozyme. Chembiolchem 2001, 2, 31-35.

487. Wolf, Y.I.; Koonin, E.V. On the origin of the translation system and the genetic code in the RNA world by means of natural selection, exaptation, and subfunctionalization. Biol.Direct 2007, 2, doi:10.1186/1745-6150-2-14.

488. Ma, W. The scenario on the origin of translation in the RNA world: In principle of replication parsimony. Biol.Direct 2010, 5, doi:10.1186/1745-6150-5-65.

489. Ibba, M.; Soll, D. Aminoacyl-tRNAs: Setting the limits of the genetic code. Genes Dev. 2004, 18, 731-738.

490. Wong, J.T. A co-evolution theory of the genetic code. Proc. Natl. Acad. Sci. USA 1975, 72 , 1909-1912.

491. Wong, J.T. Coevolution theory of the genetic code at age thirty. BioEssays 2005, 27, 416-425.

492. Easterwood, T.R.; Major, F.; Malhotra, A.; Harvey, S.C. Orientations of transfer RNA in the ribosomal A and P sites. Nucleic Acids Res. 1994, 22, 3779-3786.

493. Schmeing, T.M.; Moore, P.B.; Steitz, T.A. Structures of deacylated tRNA mimics bound to the E site of the large ribosomal subunit. RNA 2003, 9, 1345-1352.

494. Tozzi, M.G.; Camici, M.; Mascia, L.; Sgarrella, F.; Ipata, P.L. Pentose phosphates in nucleoside interconversion and catabolism. FEBS J. 2006, 273, 1089-1101.

495. Wirtz, M.; Droux, M. Synthesis of the sulfur amino acids: Cysteine and methionine. Photosynth. Res. 2005, 86, 345-362.

496. Recall that the electrogyre models the hydrogen cycle (3.1), the oxygyre models the oxygen and water cycles (3.2), the carbogyre models the carbon cycle (3.3), the phosphogyre models the phosphorus cycle (3.4), and the ribogyre and nitrogyre model the nitrogen cycle (3.5).

497. Kertesz, M.A. Riding the sulfur cycle-Metabolism of sulfonates and sulfate esters in Gram-negative bacteria. Fems Microbiol. Rev. 2000, 24, 135-175.

498. Farquhar, J.; Wu, N.P.; Canfield, D.E.; Oduro, H. Connections between sulfur cycle evolution, sulfur isotopes, sediments, and base metal sulfide deposits. Econ. Geol. 2010, 105, 509-533.

499. Philippot, P.; van Zuilen, M.; Lepot, K.; Thomazo, C.; Farquhar, J.; van Kranendonk, M.J. Early Archaean microorganisms preferred elemental sulfur, not sulfate. Science 2007, 317, 1534-1537. 
500. Turchyn, A.V.; Schrag, D.P. Oxygen isotope constraints on the sulfur cycle over the past 10 million years. Science 2004, 303, 2004-2007.

501. Takahashi, H.; Kopriva, S.; Giordano, M.; Saito, K.; Hell, R. Sulfur assimilation in photosynthetic organisms: Molecular functions and regulations of transporters and assimilatory enzymes. Annu. Rev. Plant Biol. 2011, 62, 157-184.

502. Stipanuk, M.H. Metabolism of sulfur-containing amino acids. Annu. Rev. Nutr. 1986, 6, 179-209.

503. Brosnan, J.T.; Brosnan, M.E. The sulfur-containing amino acids: An overview. J. Nutr. 2006, 136, 1636S-1640S.

504. Chatterjee, N.K.; Kerwar, S.S.; Weissbach, H. Initiation of protein synthesis in HeLa cells. Proc. Natl. Acad. Sci. USA 1972, 69, 1375-1379.

505. Han, D.X.; Wang, H.Y.; Ji, Z.L.; Hu, A.F.; Zhao, Y.F. Amino Acid Homochirality may be Linked to the Origin of Phosphate-Based Life. J. Mol. Evol. 2010, 70, 572-582.

506. Tamura, K. Origin of amino acid homochirality: Relationship with the RNA world and origin of tRNA aminoacylation. Biosystems 2008, 92, 91-98.

507. Zaher, H.S.; Green, R. Fidelity at the molecular level: Lessons from protein synthesis. Cell 2009, $136,746-762$.

508. Blommaart, E.F.; Luiken, J.J.; Meijer, A.J. Autophagic proteolysis: Control and specificity. Histochem. J. 1997, 29, 365-385.

509. Hamel, F.G.; Fawcett, J.; Bennett, R.G.; Duckworth, W.C. Control of proteolysis: Hormones, nutrients, and the changing role of the proteasome. Curr. Opin. Clin. Metab. Care 2004, 7, 255-258.

510. Kadowaki, M.; Kanazawa, T. Amino acids as regulators of proteolysis. J. Nutr. 2003, 133, 2052S-2056S.

511. Knaggs, M.; Williams, M.; Goodfellow, J.M. Protein hydration, stability and unfolding. Biochem. Soc. Trans. 1995, 23, 711-715.

512. Harari-Steinberg, O.; Chamovitz, D.A. The COP9 signalosome: Mediating between kinase signaling and protein degradation. Curr. Protein Pept. Sci. 2004, 5, 185-189.

513. Fuchs, S.Y.; Fried, V.A.; Ronai, Z. Stress-activated kinases regulate protein stability. Oncogene 1998, 17, 1483-1490.

514. Buljan, M.; Bateman, A. The evolution of protein domain families. Biochem. Soc. Trans. 2009, 37, 751-755.

515. Caetano-Anolles, G.; Wang, M.; Caetano-Anolles, D.; Mittenthal, J.E. The origin, evolution and structure of the protein world. Biochem. J. 2009, 417, 621-637.

516. Pal, C.; Papp, B.; Lercher, M.J. An integrated view of protein evolution. Nat. Rev. Genet. 2006, 7, 337-348.

517. Hausmann, C.D.; Ibba, M. Aminoacyl-tRNA synthetase complexes: Molecular multitasking revealed. FEMS Microbiol. Rev. 2008, 32, 705-721.

518. Lipman, R.S.; Hou, Y.M. Aminoacylation of tRNA in the evolution of an aminoacyl-tRNA synthetase. Proc. Natl. Acad. Sci. USA 1998, 95, 13495-13500.

519. Errington, N.; Iqbalsyah, T.; Doig, A.J. Structure and stability of the alpha-helix: Lessons for design. Methods Mol. Biol. 2006, 340, 3-26.

520. Toniolo, C.; Benedetti, E. The polypeptide 310-helix. Trends Biochem. Sci. 1991, 16, 350-353. 
521. Riek, R.P.; Graham, R.M. The elusive pi-helix. J. Struct. Biol. 2011, 173, 153-160.

522. Chou, K.C.; Pottle, M.; Nemethy, G.; Ueda, Y.; Scheraga, H.A. Structure of beta-sheets. Origin of the right-handed twist and of the increased stability of antiparallel over parallel sheets. J. Mol. Biol. 1982, 162, 89-112.

523. Armen, R.; Alonso, D.O.; Daggett, V. The role of alpha-, 3(10)-, and pi-helix in helix $\rightarrow$ coil transitions. Protein Sci. 2003, 12, 1145-1157.

524. Schutt, C.E.; Myslik, J.C.; Rozycki, M.D.; Goonesekere, N.C.; Lindberg, U. The structure of crystalline profilin-beta-actin. Nature 1993, 365, 810-816.

525. Nogales, E.; Whittaker, M.; Milligan, R.A.; Downing, K.H. High-resolution model of the microtubule. Cell 1999, 96, 79-88.

526. Strelkov, S.V.; Schumacher, J.; Burkhard, P.; Aebi, U.; Herrmann, H. Crystal structure of the human lamin A coil 2B dimer: Implications for the head-to-tail association of nuclear lamins. J. Mol. Biol. 2004, 343, 1067-1080.

527. Bhattacharjee, A.; Bansal, M. Collagen structure: The Madras triple helix and the current scenario. IUBMB Life 2005, 57, 161-172.

528. Wierzbicka-Patynowski, I.; Schwarzbauer, J.E. The ins and outs of fibronectin matrix assembly. J. Cell Sci. 2003, 116, 3269-3276.

529. Leahy, D.J.; Aukhil, I.; Erickson, H.P. 2.0 A crystal structure of a four-domain segment of human fibronectin encompassing the RGD loop and synergy region. Cell 1996, 84, 155-164.

530. Beck, K.; Hunter, I.; Engel, J. Structure and function of laminin: Anatomy of a multidomain glycoprotein. FASEB J. 1990, 4, 148-160.

531. Ali, I.; Marenduzzo, D.; Yeomans, J.M. Polymer packaging and ejection in viral capsids: Shape matters. Phys. Rev. Lett. 2006, 96, doi:10.1103/PhysRevLett.96.208102.

532. Zampighi, G.A.; Fisher, R.S. Polyhedral protein cages encase synaptic vesicles and participate in their attachment to the active zone. J. Struct. Biol. 1997, 119, 347-359.

533. Anfinsen, C.B. Studies on the reduction and re-formation of protein disulfide bonds. J. Biol. Chem. 1961, 236, 1361-1363.

534. Anfinsen, C.B. Principles that govern folding of protein chains. Science 1973, 181, 223-230.

535. Ptitsyn, O.B. A determinable but unresolved problem. FASEB J. 1996, 10, 3-4.

536. I have modeled that the aminonexus is composed of amino acids (ribogyre (3.5) and aminogyre (3.6)) that evolutionarily emerge from organic matter and oxides and are bathed in a water solution (oxygyre (3.2) and carbogyre (3.3)). Furthermore, the aminonexus is replete with electrons (electrogyre (3.1)) and frequently modified with phosphate groups (phosphogyre (3.4)).

537. Nordlund, P.; Reichard, P. Ribonucleotide reductases. Annu. Rev. Biochem. 2006, 75, 681-706.

538. Kennelly, P.J. Protein kinases and protein phosphatases in prokaryotes: A genomic perspective. FEMS Microbiol. Lett. 2002, 206, 1-8.

539. Taylor, S.S.; Kornev, A.P. Protein kinases: Evolution of dynamic regulatory proteins. Trends Biochem. Sci. 2011, 36, 65-77.

540. Kennelly, P.J. Protein phosphatases-A phylogenetic perspective. Curr. Rev. 2001, 101, 2291-2312.

541. Kwapisz, M.; Beckouet, F.; Thuriaux, P. Early evolution of eukaryotic DNA-dependent RNA polymerases. Trends Genet. 2008, 24, 211-215. 
542. Sonntag, K.C.; Darai, G. Evolution of viral DNA-dependent RNA polymerases. Virus Genes 1995, 11, 271-284.

543. Werner, F.; Grohmann, D. Evolution of multisubunit RNA polymerases in the three domains of life. Nat. Rev. Microbiol. 2011, 9, 85-98.

544. Makeyev, E.V.; Grimes, J.M. RNA-dependent RNA polymerases of dsRNA bacteriophages. Virus Res. 2004, 101, 45-55.

545. Sorrentino, S. The eight human "canonical" ribonucleases: Molecular diversity, catalytic properties, and special biological actions of the enzyme proteins. FEBS Lett. 2010, 584, 2194-2200.

546. Danchin, A. A phylogenetic view of bacterial ribonucleases. Progress Mol. Biol. Transl. Sci. 2009, $85,1-41$.

547. Evans, C.J.; Aguilera, R.J. DNase II: Genes, enzymes and function. Gene 2003, 322, 1-15.

548. Pyle, A.M. Translocation and unwinding mechanisms of RNA and DNA helicases. Annu. Rev. Biophys. 2008, 37, 317-336.

549. Tan, S.; Richmond, T.J. Eukaryotic transcription factors. Curr. Opin. Struct. Biol. 1998, 8, 41-48.

550. Reeve, J.N.; Bailey, K.A.; Li, W.T.; Marc, F.; Sandman, K.; Soares, D.J. Archaeal histones: Structures, stability and DNA binding. Biochem. Soc. Trans. 2004, 32, 227-230.

551. Johnson, L.N. The regulation of protein phosphorylation. Biochem. Soc. Trans. 2009, 37, 627-641.

552. Bayle, J.H.; Crabtree, G.R. Protein acetylation: More than chromatin modification to regulate transcription. Chem. Biol. 1997, 4, 885-888.

553. McIlhinney, R.A. The fats of life: The importance and function of protein acylation. Trends Biochem. Sci. 1990, 15, 387-391.

554. Paik, W.K.; Paik, D.C.; Kim, S. Historical review: The field of protein methylation. Trends Biochem. Sci. 2007, 32, 146-152.

555. Fang, S.; Weissman, A.M. A field guide to ubiquitylation. Cell. Mol. Life Sci. 2004, 61, 1546-1561.

556. Wilkinson, K.A.; Henley, J.M. Mechanisms, regulation and consequences of protein SUMOylation. Biochem. J. 2010, 428, 133-145.

557. Protein phosphorylation is modeled as thermodynamic relationships between the ribogyre (NTPs) and aminogyre; protein acetylation, acylation, and methylation are modeled as relationships between the carbogyre and aminogyre; and protein ubiquitylation and SUMOylation occur via aminogyre autoregulation.

558. Wendler, P.; Ciniawsky, S.; Kock, M.; Kube, S. Structure and function of the AAA+ nucleotide binding pocket. Biochim. Biophys. Acta 2011, in press.

559. Blower, M.D.; Nachury, M.; Heald, R.; Weis, K. A Rae1-containing ribonucleoprotein complex is required for mitotic spindle assembly. Cell 2005, 121, 223-234.

560. Van Hooser, A.A.; Yuh, P.; Heald, R. The perichromosomal layer. Chromosoma 2005, 114, 377-388.

561. Cole, C.N.; Scarcelli, J.J. Transport of messenger RNA from the nucleus to the cytoplasm. Curr. Opin. Cell. Biol. 2006, 18, 299-306.

562. Maniatis, T.; Reed, R. An extensive network of coupling among gene expression machines. Nature 2002, 416, 499-506. 
563. Azubel, M.; Wolf, S.G.; Sperling, J.; Sperling, R. Three-dimensional structure of the native spliceosome by cryo-electron microscopy. Mol. Cell 2004, 15, 833-839.

564. Matera, A.G.; Shpargel, K.B. Pumping RNA: Nuclear bodybuilding along the RNP pipeline. Curr. Opin. Cell. Biol. 2006, 18, 317-324.

565. Parker, R.; Sheth, U. P bodies and the control of mRNA translation and degradation. Mol. Cell 2007, 25, 635-646.

566. Erickson, S.L.; Lykke-Andersen, J. Cytoplasmic mRNP granules at a glance. J. Cell Sci. 2011, 124, 293-297.

567. Examples of these gyrapical RNA-protein complexes are the spindle ribonucleoprotein, perichromosomal sheath, nascent and nucleocytoplasmically transported mRNAs, spliceosome, Cajal bodies, and cytoplasmic P-bodies and stress granules.

568. Bowie, J.U. Solving the membrane protein folding problem. Nature 2005, 438, 581-589.

569. Pohorille, A.; Schweighofer, K.; Wilson, M.A. The origin and early evolution of membrane channels. Astrobiology 2005, 5, 1-17.

570. Debler, E.W.; Ma, Y.; Seo, H.S.; Hsia, K.C.; Noriega, T.R.; Blobel, G.; Hoelz, A. A fence-like coat for the nuclear pore membrane. Mol. Cell 2008, 32, 815-826.

571. Albrecht-Buehler, G. The iris diaphragm model of centriole and basal body formation. Cell Motil.Cytoskelet. 1990, 17, 197-213.

572. Note that each of these aminons originate and evolve in response to changes in subsumed gyrosystems; this should remind the reader of gyraxioms that dictate interconnectivity of the gyromodel $\left(\mathrm{G}_{\mathrm{IV}}, \mathrm{G}_{\mathrm{IV}-1}\right)$ : Although these phenomena-polypeptide synthesis, protein complex structure and function, and membrane insertion - can be studied independently, they cannot be understood without losing information related to the processes that precede them evolutionarily, atomically, and metabolically.

573. Walsh, C. Antibiotics: Actions, Origins, Resistance; ASM Press: Washington, DC, USA, 2003; p. 335.

574. Yim, G.; Wang, H.H.; Davies, J. Antibiotics as signalling molecules. Philos. Trans. R. Soc. Lond. Ser. B 2007, 362, 1195-1200.

575. Kleinkauf, H.; von Dohren, H. A nonribosomal system of peptide biosynthesis. Eur. J. Biochem. FEBS 1996, 236, 335-351.

576. Von Dohren, H.; Dieckmann, R.; Pavela-Vrancic, M. The nonribosomal code. Chem. Biol. 1999, 6, R273-R279.

577. Caboche, S.; Leclere, V.; Pupin, M.; Kucherov, G.; Jacques, P. Diversity of monomers in nonribosomal peptides: Towards the prediction of origin and biological activity. J. Bacteriol. 2010, 192, 5143-5150.

578. French, G.L. The continuing crisis in antibiotic resistance. Int. J. Antimicrob. Agents 2010, 36, S3-S7.

579. Shimojima, M. Biosynthesis and functions of the plant sulfolipid. Progress in Lipid Research. 2011, 50, 234-239.

580. Vollmer, W.; Blanot, D.; de Pedro, M.A. Peptidoglycan structure and architecture. FEMS Microbiol. Rev. 2008, 32, 149-167.

581. Irvine, W.M. Chemistry between the stars. Planet. Rep. 1987, 7, 6-9. 
582. Kolberg, M.; Strand, K.R.; Graff, P.; Andersson, K.K. Structure, function, and mechanism of ribonucleotide reductases. Biochim. Biophys. Acta 2004, 1699, 1-34.

583. Stubbe, J. Ribonucleotide reductases: The link between an RNA and a DNA world? Curr. Opin. Struct. Biol. 2000, 10, 731-736.

584. Forterre, P. The two ages of the RNA world, and the transition to the DNA world: A story of viruses and cells. Biochimie 2005, 87, 793-803.

585. Reichard, P. From RNA to DNA, why so many ribonucleotide reductases? Science 1993, 260, 1773-1777.

586. Watson, J.D.; Berry, A. DNA: The Secret of Life, 1st ed.; Alfred A. Knopf: New York, NY, USA, 2003; p. 446.

587. Witkowski, J.A. The Inside Story: DNA to RNA to Protein; Cold Spring Harbor Laboratory Press: Woodbury, NY, USA, 2005; p. 382.

588. Van Rompay, A.R.; Johansson, M.; Karlsson, A. Phosphorylation of nucleosides and nucleoside analogs by mammalian nucleoside monophosphate kinases. Pharmacol. Ther. 2000, 87, 189-198.

589. Chakrabarty, A.M. Nucleoside diphosphate kinase: Role in bacterial growth, virulence, cell signalling and polysaccharide synthesis. Mol. Microbiol. 1998, 28, 875-882.

590. Itzkovitz, S.; Tlusty, T.; Alon, U. Coding limits on the number of transcription factors. $B M C$ Genomics 2006, 7, doi:10.1186/1471-2164-7-239.

591. Karin, M. Too many transcription factors: Positive and negative interactions. New Biol. 1990, 2, 126-131.

592. Georges, A.B.; Benayoun, B.A.; Caburet, S.; Veitia, R.A. Generic binding sites, generic DNA-binding domains: Where does specific promoter recognition come from? FASEB J. 2010, 24, 346-356.

593. Bergman, C.M.; Pfeiffer, B.D.; Rincon-Limas, D.E.; Hoskins, R.A.; Gnirke, A.; Mungall, C.J.; Wang, A.M.; Kronmiller, B.; Pacleb, J.; Park, S.; et al. Assessing the impact of comparative genomic sequence data on the functional annotation of the Drosophila genome. Genome Biol. 2002, 3, 1-20.

594. Cameron, R.A.; Chow, S.H.; Berney, K.; Chiu, T.Y.; Yuan, Q.A.; Kramer, A.; Helguero, A.; Ransick, A.; Yun, M.; Davidson, E.H. An evolutionary constraint: Strongly disfavored class of change in DNA sequence during divergence of cis-regulatory modules. Proc. Natl. Acad. Sci. USA 2005, 102, 11769-11774.

595. Carninci, P.; Sandelin, A.; Lenhard, B.; Katayama, S.; Shimokawa, K.; Ponjavic, J.; Semple, C.A.; Taylor, M.S.; Engstrom, P.G.; Frith, M.C.; et al. Genome-wide analysis of mammalian promoter architecture and evolution. Nat. Genet. 2006, 38, 626-635.

596. Castillo-Davis, C.I. The evolution of noncoding DNA: How much junk, how much func? Trends Genet. 2005, 21, 533-536.

597. Dillon, S.C.; Dorman, C.J. Bacterial nucleoid-associated proteins, nucleoid structure and gene expression. Nat. Rev. Microbiol. 2010, 8, 185-195.

598. Thatcher, T.H.; Gorovsky, M.A. Phylogenetic analysis of the core histones H2A, H2B, H3, and H4. Nucleic Acids Res. 1994, 22, 174-179. 
599. Kato, M.; Onishi, Y.; Wada-Kiyama, Y.; Kiyama, R. Biochemical screening of stable dinucleosomes using DNA fragments from a dinucleosome DNA library. J. Mol. Biol. 2005, 350, 215-227.

600. Sollner-Webb, B.; Felsenfeld, G. A comparison of the digestion of nuclei and chromatin by staphylococcal nuclease. Biochemistry 1975, 14, 2915-2920.

601. Lucchesi, J.C. Dosage compensation in Drosophila and the "complex' world of transcriptional regulation. BioEssays 1996, 18, 541-547.

602. Winston, F.; Carlson, M. Yeast SNF/SWI transcriptional activators and the SPT/SIN chromatin connection. Trends Genet. 1992, 8, 387-391.

603. Liu, Z.; Karmarkar, V. Groucho/Tup1 family co-repressors in plant development. Trends Plant Sci. 2008, 13, 137-144.

604. Melendy, T.; Li, R. Chromatin remodeling and initiation of DNA replication. Front. Biosci. 2001, 6, D1048-D1053.

605. Friedberg, E.C. DNA damage and repair. Nature 2003, 421, 436-440.

606. Coghlan, A.; Eichler, E.E.; Oliver, S.G.; Paterson, A.H.; Stein, L. Chromosome evolution in eukaryotes: A multi-kingdom perspective. Trends Genet. 2005, 21, 673-682.

607. Feschotte, C.; Pritham, E.J. DNA transposons and the evolution of eukaryotic genomes. Annu. Rev. Genet. 2007, 41, 331-368.

608. McClintock, B. The origin and behavior of mutable loci in maize. Proc. Natl. Acad. Sci. USA 1950, 36, 344-355.

609. Stankiewicz, P.; Lupski, J.R. Genome architecture, rearrangements and genomic disorders. Trends Genet. 2002, 18, 74-82.

610. Zhao, J.; Bacolla, A.; Wang, G.; Vasquez, K.M. Non-B DNA structure-induced genetic instability and evolution. Cell. Mol. Life Sci. 2010, 67, 43-62.

611. Ghosh, A.; Bansal, M. A glossary of DNA structures from A to Z. Acta Crystallogr. Sect. D 2003, 59, 620-626.

612. Watson, J.D.; Crick, F.H. Molecular structure of nucleic acids; a structure for deoxyribose nucleic acid. Nature 1953, 171, 737-738.

613. McGhee, J.D.; Nickol, J.M.; Felsenfeld, G.; Rau, D.C. Higher order structure of chromatin: Orientation of nucleosomes within the $30 \mathrm{~nm}$ chromatin solenoid is independent of species and spacer length. Cell 1983, 33, 831-841.

614. Filipski, J.; Leblanc, J.; Youdale, T.; Sikorska, M.; Walker, P.R. Periodicity of DNA folding in higher order chromatin structures. EMBO J. 1990, 9, 1319-1327.

615. Swedlow, J.R.; Hirano, T. The making of the mitotic chromosome: Modern insights into classical questions. Mol. Cell 2003, 11, 557-569.

616. Forterre, P. The origin of viruses and their possible roles in major evolutionary transitions. Virus Res. 2006, 117, 5-16.

617. Long, M.; Betran, E.; Thornton, K.; Wang, W. The origin of new genes: Glimpses from the young and old. Nat. Rev. Genet. 2003, 4, 865-875.

618. Zhou, Q.; Wang, W. On the origin and evolution of new genes-A genomic and experimental perspective. J. Genet. Genomics 2008, 35, 639-648. 
619. Hittinger, C.T.; Carroll, S.B. Gene duplication and the adaptive evolution of a classic genetic switch. Nature 2007, 449, 677-681.

620. Ohno, S. Evolution by Gene Duplication; Springer-Verlag: Berlin, Germany and New York, NY, USA, 1970; p. 160.

621. Gilbert, W.; de Souza, S.J.; Long, M. Origin of genes. Proc. Natl. Acad. Sci. USA 1997, 94, 7698-7703.

622. Swanson, W.J. Adaptive evolution of genes and gene families. Curr. Opin. Genet. Dev. 2003, 13, 617-622.

623. Rodriguez-Trelles, F.; Tarrio, R.; Ayala, F.J. Origins and evolution of spliceosomal introns. Annu. Rev. Genet. 2006, 40, 47-76.

624. Catania, F.; Lynch, M. Where do introns come from? PLoS Biol. 2008, 6, doi:10.1371/journal. pbio.0060283.

625. Brisson, D. The directed mutation controversy in an evolutionary context. Crit. Rev. Microbiol. 2003, 29, 25-35.

626. Cairns, J.; Overbaugh, J.; Miller, S. The origin of mutants. Nature 1988, 335, 142-145.

627. Lenski, R.E.; Mittler, J.E. The directed mutation controversy and neo-Darwinism. Science 1993, 259, 188-194.

628. Cheng, J.; Kapranov, P.; Drenkow, J.; Dike, S.; Brubaker, S.; Patel, S.; Long, J.; Stern, D.; Tammana, H.; Helt, G.; et al. Transcriptional maps of 10 human chromosomes at 5-nucleotide resolution. Science 2005, 308, 1149-1154.

629. Huertas, P.; Aguilera, A. Cotranscriptionally formed DNA:RNA hybrids mediate transcription elongation impairment and transcription-associated recombination. Mol. Cell 2003, 12, 711-721.

630. Nowacki, M.; Vijayan, V.; Zhou, Y.; Schotanus, K.; Doak, T.G.; Landweber, L.F. RNA-mediated epigenetic programming of a genome-rearrangement pathway. Nature 2008, 451, 153-158.

631. Storici, F.; Bebenek, K.; Kunkel, T.A.; Gordenin, D.A.; Resnick, M.A. RNA-templated DNA repair. Nature 2007, 447, 338-341.

632. Jenkins, F.J.; Roizman, B. Site-specific mutagenesis of large DNA viral genomes. BioEssays 1986, 5, 244-247.

633. Duffy, S.; Shackelton, L.A.; Holmes, E.C. Rates of evolutionary change in viruses: Patterns and determinants. Nat. Rev. Genet. 2008, 9, 267-276.

634. Dunning Hotopp, J.C.; Clark, M.E.; Oliveira, D.C.; Foster, J.M.; Fischer, P.; Munoz Torres, M.C.; Giebel, J.D.; Kumar, N.; Ishmael, N.; Wang, S.; et al. Widespread lateral gene transfer from intracellular bacteria to multicellular eukaryotes. Science 2007, 317, 1753-1756.

635. Timmis, J.N.; Ayliffe, M.A.; Huang, C.Y.; Martin, W. Endosymbiotic gene transfer: Organelle genomes forge eukaryotic chromosomes. Nat. Rev. Genet. 2004, 5, 123-135.

636. Bergthorsson, U.; Adams, K.L.; Thomason, B.; Palmer, J.D. Widespread horizontal transfer of mitochondrial genes in flowering plants. Nature 2003, 424, 197-201.

637. Mathews, C.K. DNA precursor metabolism and genomic stability. FASEB J. 2006, 20, 1300-1314.

638. Niida, H.; Shimada, M.; Murakami, H.; Nakanishi, M. Mechanisms of dNTP supply that play an essential role in maintaining genome integrity in eukaryotic cells. Cancer Sci. 2010, 101, 2505-2509. 
639. Hakansson, P.; Hofer, A.; Thelander, L. Regulation of mammalian ribonucleotide reduction and dNTP pools after DNA damage and in resting cells. J. Biol. Chem. 2006, 281, 7834-7841.

640. Chabes, A.; Stillman, B. Constitutively high dNTP concentration inhibits cell cycle progression and the DNA damage checkpoint in yeast Saccharomyces cerevisiae. Proc. Natl. Acad. Sci. USA 2007, 104, 1183-1188.

641. Harper, J.W.; Elledge, S.J. The DNA damage response: Ten years after. Mol. Cell 2007, 28, 739-745.

642. Zou, L.; Elledge, S.J. Sensing DNA damage through ATRIP recognition of RPA-ssDNA complexes. Science 2003, 300, 1542-1548.

643. El-Hani, C.N. Between the cross and the sword: The crisis of the gene concept. Genet. Mol. Biol. 2007, 30, 297-307.

644. Tautz, D. Redundancies, development and the flow of information. BioEssays 1992, 14, 263-266.

645. Keller, E.F. The century beyond the gene. J. Biosci. 2005, 30, 3-10.

646. Biro, J.C. Seven fundamental, unsolved questions in molecular biology. Cooperative storage and bi-directional transfer of biological information by nucleic acids and proteins: An alternative to "central dogma". Med. Hypotheses 2004, 63, 951-962.

647. Falk, R. The rise and fall of dominance. Biol. Philos. 2001, 16, 285-323.

648. Ubeda, F. Why Mendelian segregation? Biochem. Soc. Trans. 2006, 34, 566-568.

649. Ayala, F.J. Darwin's greatest discovery: Design without designer. Proc. Natl. Acad. Sci. USA 2007, 104, 8567-8573.

650. Dawkins, R. The Selfish Gene, 30th Anniversary ed.; Oxford University Press: Oxford, UK and New York, NY, USA, 2006; p. 360.

651. Lombard, D.B.; Chua, K.F.; Mostoslavsky, R.; Franco, S.; Gostissa, M.; Alt, F.W. DNA repair, genome stability, and aging. Cell 2005, 120, 497-512.

652. Kolodner, R.D.; Putnam, C.D.; Myung, K. Maintenance of genome stability in Saccharomyces cerevisiae. Science 2002, 297, 552-557.

653. Orr, H.A. The genetic theory of adaptation: A brief history. Nat. Rev. Genet. 2005, 6, 119-127.

654. Visscher, P.M.; Hill, W.G.; Wray, N.R. Heritability in the genomics era-concepts and misconceptions. Nat. Rev. Genet. 2008, 9, 255-266.

655. Cobb, A.B. Cell Theory; Chelsea House: New York, NY, USA, 2011; p. 104.

656. Cano, R.J.; Borucki, M.K. Revival and identification of bacterial spores in 25- to 40-millionyear-old Dominican amber. Science 1995, 268, 1060-1064.

657. Sargent, M.G. Control of membrane protein synthesis in Bacillus subtilis. Biochim. Biophys. Acta 1975, 406, 564-574.

658. Cadenas, E.; Garland, P.B. Synthesis of cytoplasmic membrane during growth and division of Escherichia coli. Dispersive behaviour of respiratory nitrate reductase. Biochem. J. 1979, 184, 45-50.

659. Diffley, J.F. Eukaryotic DNA replication. Curr. Opin. Cell. Biol. 1994, 6, 368-372.

660. Leipe, D.D.; Aravind, L.; Koonin, E.V. Did DNA replication evolve twice independently? Nucleic Acids Res. 1999, 27, 3389-3401.

661. Harry, E.; Monahan, L.; Thompson, L. Bacterial cell division: The mechanism and its precison. Int. Rev. Cytol. 2006, 253, 27-94. 
662. Uhlmann, F. Chromosome cohesion and segregation in mitosis and meiosis. Curr. Opin. Cell. Biol. 2001, 13, 754-761.

663. Woese, C.R.; Magrum, L.J.; Fox, G.E. Archaebacteria. J. Mol. Evol. 1978, 11, 245-251.

664. Galtier, N.; Gouy, M. Molecular phylogeny of Eubacteria: A new multiple tree analysis method applied to 15 sequence data sets questions the monophyly of gram-positive bacteria. Res. Microbiol. 1994, 145, 531-541.

665. Pace, N.R. Time for a change. Nature 2006, 441, 289-289.

666. McBride, H.M.; Neuspiel, M.; Wasiak, S. Mitochondria: More than just a powerhouse. Curr. Biol. 2006, 16, R551-560.

667. Kuroiwa, T.; Kuroiwa, H.; Sakai, A.; Takahashi, H.; Toda, K.; Itoh, R. The division apparatus of plastids and mitochondria. Int. Rev. Cytol. 1998, 181, 1-41.

668. Cooper, S. On G0 and cell cycle controls. BioEssays 1987, 7, 220-223.

669. Coelho, S.M.; Peters, A.F.; Charrier, B.; Roze, D.; Destombe, C.; Valero, M.; Cock, J.M. Complex life cycles of multicellular eukaryotes: New approaches based on the use of model organisms. Gene 2007, 406, 152-170.

670. Martin, W.F.; Müller, M. Origin of Mitochondria and Hydrogenosomes; Springer: New York, NY, USA, 2007.

671. Yoon, H.S.; Hackett, J.D.; Pinto, G.; Bhattacharya, D. The single, ancient origin of chromist plastids. Proc. Natl. Acad. Sci. USA 2002, 99, 15507-15512.

672. Mahler, H.R.; Raff, R.A. Evolutionary origin of mitochondrion-non-symbiotic model. Int. Rev. Cytol. 1975, 43, 1-124.

673. Sagan, L. On origin of mitosing cells. J. Theor. Biol. 1967, 14, 225-274.

674. Cotton, D.W. Intimate relations: The serial endosymbiotic theory of the origin of eukaryotes. J. Pathol. 1993, 169, 189-190.

675. Martin, W.; Muller, M. The hydrogen hypothesis for the first eukaryote. Nature 1998, 392, $37-41$.

676. Torres, E.M.; Williams, B.R.; Amon, A. Aneuploidy: Cells losing their balance. Genetics 2008, 179, 737-746.

677. Chen, X.J.; Clark-Walker, G.D. The petite mutation in yeasts: 50 years on. Int. Rev. Cytol. 2000, 194, 197-238.

678. Lindemann, S.; Gawaz, M. The active platelet: Translation and protein synthesis in an anucleate cell. Semin. Thromb. Hemost. 2007, 33, 144-150.

679. Palis, J.; Segel, G.B. Developmental biology of erythropoiesis. Blood Rev. 1998, 12, 106-114.

680. Nagata, S. DNA degradation in development and programmed cell death. Annu. Rev. Immunol. 2005, 23, 853-875.

681. Counis, M.F.; Chaudun, E.; Arruti, C.; Oliver, L.; Sanwal, M.; Courtois, Y.; Torriglia, A. Analysis of nuclear degradation during lens cell differentiation. Cell Death Differ. 1998, 5, 251-261.

682. Blagosklonny, M.V.; Pardee, A.B. The restriction point of the cell cycle. Cell Cycle 2002, 1, 103-110.

683. Donjerkovic, D.; Scott, D.W. Regulation of the G1 phase of the mammalian cell cycle. Cell Res. 2000, 10, 1-16. 
684. Laskey, R.A.; Fairman, M.P.; Blow, J.J. S phase of the cell cycle. Science 1989, 246, 609-614.

685. Cuddihy, A.R.; O’Connell, M.J. Cell-cycle responses to DNA damage in G2. Int. Rev. Cytol. 2003, 222, 99-140.

686. Gorbsky, G.J. Cell cycle checkpoints: Arresting progress in mitosis. BioEssays 1997, 19, 193-197.

687. Chang, F.; Nurse, P. Finishing the cell cycle: Control of mitosis and cytokinesis in fission yeast. Trends Genet. 1993, 9, 333-335.

688. Rabinowitz, M. Studies on the cytology and early embryology of the egg of Drosophila melanogaster. J. Morphol. 1941, 69, 1-49.

689. Edgar, B.A.; O'Farrell, P.H. Genetic control of cell division patterns in the Drosophila embryo. Cell 1989, 57, 177-187.

690. Lloyd, D.; Poole, R.K.; Edwards, S.W. The Cell Division Cycle: Temporal Organization and Control of Cellular Growth and Reproduction; Academic Press: New York, NY, USA, 1982; p. 523.

691. Nasmyth, K. Evolution of the cell-cycle. Philos. Trans. R. Soc. Lond. Ser. B 1995, 349, 271-281.

692. Haeckel, E. Art Forms in Nature; Dover Publications: New York, NY, USA, 1974.

693. Biggs, D.S. 3D deconvolution microscopy. Curr. Protoc. Cytom. 2010, Chapter 12, 12.19:1-12.19:20.

694. Diaspro, A.; Chirico, G.; Collini, M. Two-photon fluorescence excitation and related techniques in biological microscopy. Q. Rev. Biophys. 2005, 38, 97-166.

695. Horwitz, R. Cell biology as the centuries change-About as good as it gets. J. Cell Sci. 2000, 113, 906-908.

696. Moon, J.; Hake, S. How a leaf gets its shape. Curr. Opin. Plant Biol. 2011, 14, 24-30.

697. Stolz, J.F.; Franks, J. Flat laminated microbial mat communities. Earth Sci. Rev. 2009, 96, 163-172.

698. Jelsbak, L.; Sogaard-Andersen, L. Cell behavior and cell-cell communication during fruiting body morphogenesis in Myxococcus xanthus. J. Microbiol. Methods 2003, 55, 829-839.

699. Tice, M.M.; Lowe, D.R. Photosynthetic microbial mats in the 3,416-Myr-old ocean. Nature 2004, 431, 549-552.

700. Lopez-Garcia, P.; Moreira, D.; Douzery, E.; Forterre, P.; van Zuilen, M.; Claeys, P.; Prieur, D. Ancient fossil record and early evolution (ca. 3.8 to $0.5 \mathrm{Ga}$ ). Earth Moon Planets 2006, 98, 247-290.

701. Thery, M.; Bornens, M. Cell shape and cell division. Curr. Opin. Cell. Biol. 2006, 18, 648-657.

702. Cabeen, M.T.; Jacobs-Wagner, C. Bacterial cell shape. Nat. Rev. Microbiol. 2005, 3, 601-610.

703. Pajerowski, J.D.; Dahl, K.N.; Zhong, F.L.; Sammak, P.J.; Discher, D.E. Physical plasticity of the nucleus in stem cell differentiation. Proc. Natl. Acad. Sci. USA 2007, 104, 15619-15624.

704. Guo, X.; Goess1, E.; Jin, G.; Collie-Duguid, E.S.; Cassidy, J.; Wang, W.; O’Brien, V. Cell cycle perturbation and acquired 5-fluorouracil chemoresistance. Anticancer Res. 2008, 28, 9-14.

705. Taraschi, T.F.; Parashar, A.; Hooks, M.; Rubin, H. Perturbation of red cell membrane structure during intracellular maturation of Plasmodium falciparum. Science 1986, 232, 102-104.

706. Powell, C.D.; Quain, D.E.; Smart, K.A. Chitin scar breaks in aged Saccharomyces cerevisiae. Microbiology 2003, 149, 3129-3137. 
707. For example, the yeast bud scar, an end result of cell division, is a near-perfect circle of chitin on the cell surface; please note how the bud scar mirrors the craters on celestial surfaces (3.2).

708. Williams, G.C. Pleiotropy, natural selection and the evolution of senescence. Evolution 1957, 11, 398-411.

709. Lane, N. Power, Sex, Suicide: Mitochondria and the Meaning of Life; Oxford University Press: Oxford, UK and New York, NY, USA, 2005; p. 354.

710. Clark, W.R. A Means to an End: The Biological Basis of Aging and Death; Oxford University Press: New York, NY, USA, 1999; p. 234.

711. Hay, M.E.; Rasher, D.B. Coral reefs in crisis: Reversing the biotic death spiral. F1000 Biol. Rep. 2010, 2, doi:10.3410/B2-71.

712. Vesteg, M.; Krajcovic, J. On the origin of meiosis and sex. Riv. Biol. 2007, 100, 147-161.

713. Bernstein, H.; Byerly, H.C.; Hopf, F.A.; Michod, R.E. Origin of sex. J. Theor. Biol. 1984, 110, 323-351.

714. Kondrashov, A.S. The asexual ploidy cycle and the origin of sex. Nature 1994, 370, 213-216.

715. Otto, S.P.; Mable, B.K. The evolution of life cycles with haploid and diploid phases. BioEssays 1998, 20, 453-462.

716. Rodrigues, P.; Limback, D.; McGinnis, L.K.; Plancha, C.E.; Albertini, D.F. Oogenesis: Prospects and challenges for the future. J. Cell. Physiol. 2008, 216, 355-365.

717. Verlhac, M.-H.; Villeneuve, A. Oogenesis: The Universal Process; Wiley: Hoboken, NJ, USA, 2010.

718. Moore, I.T.; Lerner, J.P.; Lerner, D.T.; Mason, R.T. Relationships between annual cycles of testosterone, corticosterone, and body condition in male red-spotted garter snakes, Thamnophis sirtalis concinnus. Physiol. Biochem. Zool. 2000, 73, 307-312.

719. Tricas, T.C.; Maruska, K.P.; Rasmussen, L.E. Annual cycles of steroid hormone production, gonad development, and reproductive behavior in the Atlantic stingray. Gen. Comp. Endocrinol. 2000, 118, 209-225.

720. Hines, G.A.; Watts, S.A.; Sower, S.A.; Walker, C.W. Sex steroid levels in the testes, ovaries, and pyloric caeca during gametogenesis in the sea star Asterias vulgaris. Gen. Comp. Endocrinol. 1992, $87,451-460$.

721. Irianni, F.; Hodgen, G.D. Mechanism of ovulation. Endocrinol. Metab. Clin. N. Am. 1992, 21, 19-38.

722. Chabbert Buffet, N.; Djakoure, C.; Maitre, S.C.; Bouchard, P. Regulation of the human menstrual cycle. Front. Neuroendocrinol. 1998, 19, 151-186.

723. These cycles are nested (androgens are modeled by the carbogyre (3.3) and both lutenizing hormone and follicle-stimulating hormone are modeled by the aminogyre (3.5)) within the dipand hapcellulogyre.

724. Mayr, E. Weismann and evolution. J. Hist. Biol. 1985, 18, 295-329.

725. Gregory, T.R. The Evolution of the Genome; Elsevier Academic: Burlington, MA, USA, 2005; p. 740.

726. Cavalier-Smith, T. Nuclear volume control by nucleoskeletal DNA, selection for cell volume and cell growth rate, and the solution of the DNA C-value paradox. J. Cell Sci. 1978, 34, 247-278. 
727. Ozbek, S.; Balasubramanian, P.G.; Chiquet-Ehrismann, R.; Tucker, R.P.; Adams, J.C. The evolution of extracellular matrix. Mol. Biol. Cell 2010, 21, 4300-4305.

728. Huxley-Jones, J.; Pinney, J.W.; Archer, J.; Robertson, D.L.; Boot-Handford, R.P. Back to basics - How the evolution of the extracellular matrix underpinned vertebrate evolution. Int. J. Exp. Pathol. 2009, 90, 95-100.

729. Bereiter-Hahn, J.; Matoltsy, A.G.; Richards, K.S. Biology of the Integument; Springer-Verlag: Berlin, Germany and New York, NY, USA, 1984.

730. Gorb, S. Functional Surfaces in Biology; Springer: Dordrecht, The Netherlands, 2009; p. 678.

731. Findlay, G.H.; Harris, W.F. The topology of hair streams and whorls in man, with an observation on their relationship to epidermal ridge patterns. Am. J. Phys. Anthropol. 1977, 46, 427-437.

732. Williams, A. Spiral growth of the laminar shell of the brachiopod Crania. Calcif. Tissue Res. 1970, 6, 11-19.

733. Loza-Correa, M.; Gomez-Valero, L.; Buchrieser, C. Circadian clock proteins in prokaryotes: Hidden rhythms? Front. Microbiol. 2010, 1, doi:10.3389/fmicb.2010.00130.

734. Panda, S.; Hogenesch, J.B.; Kay, S.A. Circadian rhythms from flies to human. Nature 2002, 417, 329-335.

735. Park, D.H.; Somers, D.E.; Kim, Y.S.; Choy, Y.H.; Lim, H.K.; Soh, M.S.; Kim, H.J.; Kay, S.A.; Nam, H.G. Control of circadian rhythms and photoperiodic flowering by the Arabidopsis GIGANTEA gene. Science 1999, 285, 1579-1582.

736. Rothenfluh, A.; Abodeely, M.; Price, J.L.; Young, M.W. Isolation and analysis of six timeless alleles that cause short- or long-period circadian rhythms in Drosophila. Genetics 2000, 156, 665-675.

737. Cyran, S.A.; Buchsbaum, A.M.; Reddy, K.L.; Lin, M.C.; Glossop, N.R.; Hardin, P.E.; Young, M.W.; Storti, R.V.; Blau, J. vrille, Pdp1, and dClock form a second feedback loop in the Drosophila circadian clock. Cell 2003, 112, 329-341.

738. Gonze, D.; Leloup, J.C.; Goldbeter, A. Theoretical models for circadian rhythms in Neurospora and Drosophila. Comptes Rendus Acad. Sci. Ser. III 2000, 323, 57-67.

739. Yang, Q.; Pando, B.F.; Dong, G.; Golden, S.S.; van Oudenaarden, A. Circadian gating of the cell cycle revealed in single cyanobacterial cells. Science 2010, 327, 1522-1526.

740. Slavov, N.; Botstein, D. Coupling among growth rate response, metabolic cycle, and cell division cycle in yeast. Mol. Biol. Cell 2011, 22, 1997-2009.

741. Karsenti, E. Self-organization in cell biology: A brief history. Nat. Rev. Mol. Cell Biol. 2008, 9, 255-262.

742. Sapp, J. Microbial Phylogeny and Evolution: Concepts and Controversies; Oxford University Press: New York, NY, USA, 2005; p. 362.

743. Franc, N.C. Phagocytosis of apoptotic cells in mammals, caenorhabditis elegans and Drosophila melanogaster: Molecular mechanisms and physiological consequences. Front. Biosci. 2002, 7 , d1298-1313.

744. Soldati, T.; Schliwa, M. Powering membrane traffic in endocytosis and recycling. Nat. Rev. Mol. Cell Biol. 2006, 7, 897-908.

745. Black, S. A theory on the origin of life. Adv. Enzymol. Relat. Areas Mol. Biol. 1973, 38, 193-234. 
746. Wolkenhauer, O.; Hofmeyr, J.H.S. A contribution towards a theory of living cells. At-Automatisierungstechnik 2008, 56, 225-232.

747. Olson, C.B. A theory of the origin of life. Orig. Life Evol. Biosph. 1981, 11, 353-368.

748. Lucido, G. Life out of magma: A new theory for the origin of life. Nuovo Cim. Della Soc. Ital. Fis. D 1998, 20, 2575-2591.

749. Snooks, G.D. The origin of life on earth: A new general dynamic theory. Space Life Sci. 2005, 36, 226-234.

750. Martin, W.; Russell, M.J. On the origins of cells: A hypothesis for the evolutionary transitions from abiotic geochemistry to chemoautotrophic prokaryotes, and from prokaryotes to nucleated cells. Philos. Trans. R. Soc. Lond. Ser. B 2003, 358, 59-83.

751. Kennedy, D. 125. Science 2005, 309, 15.

752. Kennedy, D.; Norman, C. What don't we know? Science 2005, 309, doi:10.1126/ science.309.5731.75.

753. Beyond the standard model. Eff. Field Theor. Flavor Phys. 2004, 203, 157-166.

754. Gold, T. The Deep Hot Biosphere; Copernicus: New York, NY, USA, 1999; p. 235.

755. Asimov, I. Fact and Fancy, 1st ed.; Doubleday: Garden City, NY, USA, 1962; p. 264.

756. Blackburn, G.M.; Bowler, M.W.; Cliff, M.J.; Waltho, J.P. Why did Nature select phosphate for its dominant roles in biology? New J. Chem. 2010, 34, 784-794.

757. Lahav, N.; Nir, S.; Elitzur, A.C. The emergence of life on Earth. Progress Biophys. Mol. Biol. 2001, 75, 75-120.

758. Jukes, T.H. Possible evolutionary steps in the genetic code. Biochem. Biophys. Res. Commun. 1982, 107, 225-228.

759. Osawa, S. Evolution of the Genetic Code; Oxford University Press: Oxford: New York, NY, USA, 1995; p. 205.

760. Goldman, A.D.; Samudrala, R.; Baross, J.A. The evolution and functional repertoire of translation proteins following the origin of life. Biol. Direct 2010, 5, doi:10.1186/1745-6150-5-15.

761. Central dogma reversed. Nature 1970, 226, 1198-1199.

762. Wag the dogma. Nat. Genet. 2002, 30, 343-344.

763. Chaisson, E.J. The cosmic environment for the growth of complexity. Biol. Syst. 1998, 46, 13-19.

764. Ball, P. Physics: Quantum all the way. Nature 2008, 453, 22-25.

765. Waldrop, M.M. Complexity: The Emerging Science at the Edge of Order and Chaos; Simon \& Schuster: New York, NY, USA, 1992; p. 380.

766. Lewin, R. Complexity: Life at the Edge of Chaos, 2nd ed.; University of Chicago Press: Chicago, IL, USA, 1999; p. 234.

767. Ellis, N.C.; Larsen-Freeman, D.; Research Club in Language Learning (Ann Arbor Mich.), Language as a Complex Adaptive System; Wiley-Blackwell: Chichester, West Sussex, UK and Malden, MA, USA, 2009; p. 275.

768. Armstrong, D.M. What Is a Law of Nature? Cambridge University Press: Cambridge Cambridgeshire, MA and New York, NY, USA, 1983; p. 180.

769. Lange, M. Laws and Lawmakers: Science, Metaphysics, and the Laws of Nature; Oxford University Press: Oxford, UK and New York, NY, USA, 2009; p. 257. 
770. Morowitz, H.J. Energy Flow in Biology; Biological Organization as a Problem in Thermal Physics; Academic Press: New York, NY, USA, 1968; p. 179.

771. Ferris, J.P.; Hill, A.R., Jr.; Liu, R.; Orgel, L.E. Synthesis of long prebiotic oligomers on mineral surfaces. Nature 1996, 381, 59-61.

772. Bohr, N.; Rosenfeld, L. Collected Works; North-Holland Publishing Co.: Amsterdam, Holland, 1972.

773. Sciama, D.W. The Unity of the Universe, 1st ed.; Doubleday: Garden City, NY, USA, 1959; p. 228.

774. Weizsäcker, C.F. The Unity of Nature; Farrar Straus Giroux: New York, NY, USA, 1980; p. 406.

775. Weinberg, S. Dreams of a Final Theory, 1st Vintage Books ed.; Vintage Books: New York, NY, USA, 1994; p. 340.

776. Prigogine, I.; Stengers, I. The End of Certainty: Time, Chaos, and the New Laws of Nature, 1st Free Press ed.; Free Press: New York, NY, USA, 1997; p. 228.

777. Wallace, D.F. Everything and More: A Compact History of Infinity, 1st ed.; Atlas Book: New York, NY, USA, 2003; p. 319.

778. Rucker, R. Infinity and the Mind: The Science and Philosophy of the Infinite; Princeton University Press: Princeton, NJ, USA, 1995; p. 342.

779. Blum, H.F. Time's Arrow and Evolution, 2nd ed.; Harper: New York, NY, USA, 1962; p. 220.

780. Wacey, D.; Kilburn, M.R.; Saunders, M.; Cliff, J.; Brasier, M.D. Microfossils of sulphur-metabolizing cells in 3.4-billion-year-old rocks of Western Australia. Nat. Geosci. 2011, 4, 698-702.

781. Eddington, A.S. The Nature of the Physical World; University Press: Cambridge, UK, 1928; p. 361.

782. Williams, G.C. Adaptation and Natural Selection; a Critique of Some Current Evolutionary Thought; Princeton University Press: Princeton, NJ, USA, 1966; p. 307.

783. Cannon, W.B. The Wisdom of the Body; W.W. Norton \& Company: New York, NY, USA, 1939; p. 333.

784. Muller, G.B.; Wagner, G.P. Novelty in evolution-Restructuring the concept. Annu. Rev. Ecol. Syst. 1991, 22, 229-256.

785. Darwin, C. The Origin of Species: Complete and Fully Illustrated; Gramercy Books: New York, NY, USA, 1979; p. 460.

786. Bak, P. How Nature Works: The Science of Self-Organized Criticality; Copernicus: New York, NY, USA, 1996; p. 212.

787. Gould, S.J.; Eldredge, N. Punctuated equilibrium comes of age. Nature 1993, 366, 223-227.

788. Henderson, L.J. The Fitness of the Environment; an Inquiry into the Biological Significance of the Properties of Matter; P. Smith: Gloucester, MA, USA, 1970; p. 317.

789. Vernadsky, V.I. The Biosphere; Copernicus: New York, NY, USA, 1998; p. 192.

790. Cleland, C.E.; Chyba, C.F. Defining 'life'. Orig. Life Evol. Biosph. 2002, 32, 387-393.

791. Des Marais, D.J.; Walter, M.R. Astrobiology: Exploring the origins, evolution, and distribution of life in the Universe. Annu. Rev. Ecol. Syst. 1999, 30, 397-420.

792. Javaux, E.J.; Dehant, V. Habitability: From stars to cells. Astron. Astrophys. Rev. 2010, 18, 383-416. 
793. Horneck, G. Exobiology, the study of the origin, evolution and distribution of life within the context of cosmic evolution: A review. Planet. Space Sci. 1995, 43, 189-217.

794. Monod, J. Chance and Necessity; an Essay on the Natural Philosophy of Modern Biology, 1st American ed.; Knopf: New York, NY, USA, 1971; p. 198.

795. Aristotle; Lawson-Tancred, H. Metaphysics; Penguin Books: London, UK and New York, NY, USA, 1998; p. 459.

796. Bachelard, G. The Formation of the Scientific Mind: A Contribution to a Psychoanalysis of Objective Knowledge; Clinamen Press Ltd.: Manchester, UK, 2006; p. 266.

797. Kuhn, T.S. The Structure of Scientific Revolutions; University of Chicago Press: Chicago, IL, USA, 1962; p. 172.

798. Strohman, R.C. The coming Kuhnian revolution in biology. Nat. Biotechnol. 1997, 15, 194-200.

799. Stent, G.S.; American Museum of Natural History. The Coming of the Golden Age; a View of the End of Progress, 1st ed.; Published for the American Museum of Natural History by the Natural History Press: Garden City, NY, USA, 1969; p. 146.

800. Horgan, J. The End of Science: Facing the Limits of Knowledge in the Twilight of the Scientific Age; Abacus: London, UK, 1998; p. 324.

(C) 2012 by the author; licensee MDPI, Basel, Switzerland. This article is an open access article distributed under the terms and conditions of the Creative Commons Attribution license (http://creativecommons.org/licenses/by/3.0/). 